\title{
Measurements of multi vector boson production processes at the LHC
}

DOI:

10.1016/j.ppnp.2019.06.002

\section{Document Version}

Accepted author manuscript

Link to publication record in Manchester Research Explorer

\section{Citation for published version (APA):}

Oh, A. (2019). Measurements of multi vector boson production processes at the LHC. Progress in Particle and Nuclear Physics, 103708. https://doi.org/10.1016/j.ppnp.2019.06.002

\section{Published in:}

Progress in Particle and Nuclear Physics

\section{Citing this paper}

Please note that where the full-text provided on Manchester Research Explorer is the Author Accepted Manuscript or Proof version this may differ from the final Published version. If citing, it is advised that you check and use the publisher's definitive version.

\section{General rights}

Copyright and moral rights for the publications made accessible in the Research Explorer are retained by the authors and/or other copyright owners and it is a condition of accessing publications that users recognise and abide by the legal requirements associated with these rights.

\section{Takedown policy}

If you believe that this document breaches copyright please refer to the University of Manchester's Takedown Procedures [http://man.ac.uk/04Y6Bo] or contact uml.scholarlycommunications@manchester.ac.uk providing relevant details, so we can investigate your claim.

\section{OPEN ACCESS}




\title{
Measurements of multi vector boson production processes at the LHC
}

\author{
A. Oh, ${ }^{1}$ \\ ${ }^{1}$ School of Physics and Astronomy, University of Manchester, UK
}

May 30, 2019

\begin{abstract}
Standard model processes with multi-boson final states provide a unique testing ground for the electroweak sector of the standard model and allow to set limits on generic model extensions to constrain the scale of new particles and interactions. The experiments at the Large Hadron Collider at CERN followed a comprehensive program to determine the properties of many accessible multiboson processes at different collision energies in run-1 and run-2. This review discusses the current status of multi-boson measurements at the LHC.
\end{abstract}

\section{Introduction}

The standard model of elementary particle physics describes the particles and interactions that are known to us today, and that constitute the universe. A plethora of experimental results seem to confirm the validity of the standard model - all predicted particles have been discovered and the precision calculations of scattering processes are consistent with the experimental observations. However, the standard model cannot be regarded as complete. For instance it cannot explain the large matter antimatter asymmetry we observe in the universe, nor does it accommodate a candidate for dark matter. In it's current form the standard model is based on the $S U_{C}(3) \otimes S U_{L}(2) \otimes U_{Y}(1)$ gauge group. The strong interaction of gluons and quarks is described by the $S U_{C}(3)$ gauge group of quantum chromo dynamics (QCD). The carrier of the weak force, the $\mathrm{W}$ and $\mathrm{Z}$ bosons, are massive spin- 1 particles (vector bosons) and represented by $S U_{L}(2)$. The gauge group $U_{Y}(1)$ is associated to the photon, carrier of the electromagnetic force. The model of electroweak interactions unifies the electromagnetic and the weak force into one quantum field gauge theory. The electroweak (EWK) Lagrangian can be written as

$$
\mathcal{L}_{E W}=\mathcal{L}_{\text {boson }}+\mathcal{L}_{\text {fermion }}+\mathcal{L}_{\text {higgs }}+\mathcal{L}_{\text {yukawa }}
$$

with $\mathcal{L}_{\text {boson }}$ the kinetic and self-interaction term of bosons, $\mathcal{L}_{\text {fermion }}$ the kinetic term describing the interaction of fermions with bosons, $\mathcal{L}_{\text {higgs }}$ the Higgs term which generates the gauge boson masses and coupling to the Higgs field, and the Yukawa term $\mathcal{L}_{\text {yukawa }}$ formalising the interaction between the fermion and Higgs field. The term $\mathcal{L}_{\text {boson }}$ allows the existence of vertices with multiple gauge bosons and hence contributes to the production of these final states.

The study of the production of multiple vector bosons has been a major effort since colliders with sufficient energy to produce final states with more than one massive vector boson have become available. The first observation of $W^{ \pm}$-pair production was made by the experiments at the Large Electron Positron 
Table 1: Basic LHC performance figures for run-1 and run-2 [11, 12]. The peak and integrated luminosity for run-2 reflects the state of data as of June 2018, the expected integrated luminosity is given in brackets.

\begin{tabular}{c|c|c|c|c}
\hline Run & $\sqrt{s}[\mathrm{GeV}]$ & Period & Peak $\mathcal{L}\left[10^{34} \mathrm{~cm}^{-2} \mathrm{~s}^{-1}\right]$ & Delivered $\int \mathcal{L} \mathrm{d} t\left[\mathrm{fb}^{-1}\right]$ \\
\hline 1 & 7 & $2010-2011$ & 0.35 & 5.5 \\
1 & 8 & $2012-2014$ & 0.77 & 22.8 \\
2 & 13 & $2015-2018$ & 2.2 & 160
\end{tabular}

collider (LEP) at CERN [1] when the centre-of-mass energy of LEP-2 was raised above the production threshold and in steps up to a maximum of $\sqrt{s}=208 \mathrm{GeV}$. The first precision measurements of doubly resonant $W^{ \pm}$-pair and $Z$-pair production allowed to verify the structure of the EWK self-interaction. The precision data on doubly resonant $W^{ \pm}$-pair production proved the existence of the $W^{+} W^{-} Z$ triple gauge boson vertex and therefore the self-interaction of massive vector bosons as described by $\mathcal{L}_{\text {boson }}$. The experiments at the Tevatron collider also contributed to the knowledge on di-boson final states and set competitive limits on new physics interactions due to the higher collision energy of $1.96 \mathrm{TeV}$, as well as discovering predicted di-boson final states like $W^{ \pm} Z$ production $[2,3]$, that were not accessible at LEP.

With the advent of the Large Hadron Collider (LHC) precision studies of multi-boson production processes became accessible, surpassing quickly the accuracy of similar studies performed at the colliders of the previous generation, i.e. LEP and the Tevatron, due to the increased collision energy. The LHC [4] was designed with the mission to find the missing experimental evidence for the elusive Higgs boson predicted by the standard model. A null result, i. e. no evidence of the Higgs boson in the expected mass range compatible with electroweak precision data [5], would have necessarily invalidated the standard model in its current form. However, with the announcement of the discovery of a particle consistent with the Higgs boson on the 4th July 2012 by the ATLAS and CMS Collaborations [6, 7], the LHC and its experiments have confirmed beautifully the standard model Higgs picture. The discovery of the last missing standard model particle concluded a very successful run-1 program of the LHC, with a collision energy of $\sqrt{s}=8 \mathrm{TeV}$. Not only was the Higgs particle discovered, but also many hitherto unaccessible processes became visible for the first time, amongst them the production of final states with up to three gauge bosons $[8,9]$. Many run-1 studies of multi-boson final states are still setting the benchmark, as will be discussed later. For an excellent review of run-1 multi-boson results see also [10].

After the end of run-1 in December 2012 the LHC undertook a major consolidation effort for two years to be able to operate at design luminosity and collision energy. Run-2 started in 2015 with an increased collision energy of $\sqrt{s}=13 \mathrm{TeV}$ and LHC reached in 2018 a peak luminosity above $2 \cdot 10^{34} \mathrm{~cm}^{-2} \mathrm{~s}^{-1}$, twice the initial design value. Run-2 ended in December 2018, followed by an consolidation period of 2 years to upgrade the injector complex and various other parts of the LHC to be able to reach $\sqrt{s}=14 \mathrm{TeV}$ and higher luminosities. Table 1 breaks down the periods of LHC operation and the approximately delivered luminosities at each operating points. The actual usable data-sets recorded by LHC experiments are typically around $90 \%$ of the delivered luminosities. Losses occur due to operational issues of detector components affecting the data quality and limitations in the trigger and data acquisition systems.

The rich data-sets delivered in run-1 and run-2 allow to do precision studies of the standard model and extend the searches for particles and interaction outside the standard model. This review focusses on the production processes of multiple vector bosons and the precision results hitherto available from the experimental collaboration at the LHC. First analyses of run-2 data have become available but the bulk of results is expected only some time after run-2 is completed to take full advantage of the integrated luminosity. Thus the majority of results discussed here are obtained from run-1 data. 
The outline of the discussion is as follows: In Section 2 a brief overview of the theory of multi-boson interactions in the SM and possible extensions to describe new phenomena is given. The experimental methods and challenges will be discussed in Section 3. A discussion of experimental results is given in Section 4, with sub-section discussing inclusive di-boson, tri-boson, and exclusive production. Finally, a summary is given in Section 5 .

\section{Phenomenology}

The self-coupling of vector-bosons is part of the standard model Lagrangian. The expanded expression, short handed $L_{\text {boson }}$ in Section 1 is given in Equation 1 following the convention in [13] for the case of three gauge bosons, $W^{ \pm} W^{ \pm} Z$ and $W^{ \pm} W^{ \pm} \gamma$, where $W^{\mu}$ stands for the $W^{-}$field, $Z^{\mu}$ is the $Z$ field, and $A^{\mu}$ represents the photon, $W_{\mu \nu}=\delta_{\mu} W_{\nu}-\delta_{\nu} W_{\mu}, V_{\mu \nu}=\delta_{\mu} V_{\nu}-\delta_{\nu} V_{\mu}$ for $V \in\{A, Z\}$, and $\left(A \overleftrightarrow{\delta_{\mu}} B\right)=$ $A\left(\delta_{\mu} B\right)-\left(\delta_{\mu} A\right) B$; the coupling strength are given by $g_{W^{ \pm} W^{ \pm} Z}=e \cot \theta_{W}$ and $g_{W^{ \pm} W^{ \pm} \gamma}=e$. Other combination of three vector bosons are excluded by either charge conservation or absence of neutral triple gauge coupling vertices in the SM.

$$
\mathcal{L}_{V V V}=i g_{W W Z}\left[W_{\mu}^{\dagger} \nu W^{\mu} Z^{\nu}-W_{\mu}^{\dagger} Z_{\nu} W^{\mu \nu}+W_{\mu}^{\dagger} W_{\nu} Z^{\mu \nu}\right]+i g_{W W \gamma}\left[W_{\mu}^{\dagger} \nu W^{\mu} A^{\nu}-W_{\mu}^{\dagger} A_{\nu} W^{\mu \nu}+W_{\mu}^{\dagger} W_{\nu} A^{\mu \nu}\right]
$$

At tree level these vertices appear in di-boson production and in vector boson fusion processes as depicted in the Feynman diagrams (a) and (b) in Figure 1. In the case of four vector boson vertices the allowed combination, constrained by charge conservation and the absence of neutral vertices in the SM, are $W^{ \pm} W^{ \pm} W^{ \pm} W^{ \pm}, W^{ \pm} W^{ \pm} Z Z, W^{ \pm} W^{ \pm} Z \gamma, W^{ \pm} W^{ \pm} \gamma \gamma$.

Similarly, an expression for the coupling of four vector bosons can be derived as shown in Equation 3 with $g_{W W W W}=g^{2}, g_{W W Z Z}=g^{2} \cos ^{2} \theta_{W}, g_{W W Z \gamma}=g^{2} \sin \theta_{W} \cos \theta_{W}$, and $g_{W W \gamma \gamma}=e^{2}$.

$$
\begin{aligned}
\mathcal{L}_{V V V V}= & \frac{g_{W W W W}}{2}\left[W^{\dagger \mu} W^{\nu} W_{\mu}^{\dagger} W_{\nu}-\left(W^{\dagger \mu} W_{\mu}\right)^{2}\right] \\
& +g_{W W Z Z}\left[Z^{\mu} Z^{\nu} W_{\mu}^{\dagger} W_{\nu}-Z^{\mu} Z_{\mu} W^{\dagger \nu} W_{\nu}\right] \\
& +g_{W W Z \gamma}\left[A^{\mu} Z^{\nu}\left(W_{\mu}^{\dagger} W_{\nu}+W_{\mu} W_{\nu}^{\dagger}\right)-2 A^{\mu} Z_{\mu} W^{\dagger \nu} W_{\nu}\right] \\
& +g_{W W \gamma \gamma}\left[A^{\mu} A^{\nu} W_{\mu}^{\dagger} W_{\nu}-A^{\mu} A_{\mu} W^{\dagger \nu} W_{\nu}\right]
\end{aligned}
$$

The interference of $s$-channel production with $t$ and $u$-channel production modes ensures good high energy behaviour, i.e. the scattering amplitude stays finite and unitarity is conserved also for high parton collision energies, $\sqrt{\hat{s}}$. Conversely, if the coupling of the $s$-channel process differs slightly from the value prescribed by the electroweak SM, observables like cross section and polarisation states will differ quite significantly with higher $\sqrt{\hat{s}}$. The multi-boson production process can thus precisely test the validity of the SM electroweak gauge structure. Deviations from the SM prediction could be caused by new particles coupling to the electroweak sector as e.g. Randal Sundrum gravitons [14] or heavy vector triplets [15]. Direct searches look for new particles appearing as resonances in the invariant mass spectrum of the di-boson system and an extensive program is followed by the LHC experiments to cover a wide range of models; for a recent review see [16].

\subsection{Effective Field Theory and anomalous couplings}

Another way to look for new particles are indirect searches, which are sensitive to higher mass scales than the direct searches can access. Here the hypothetical existence of a new particle that interacts in some way with SM particles manifest itself in subtle deviations of kinematic observables from the SM expectation through e.g. loop contributions or very wide resonances that escape direct detection. A generalised framework to quantify the new physics contribution is the effective field theory approach 


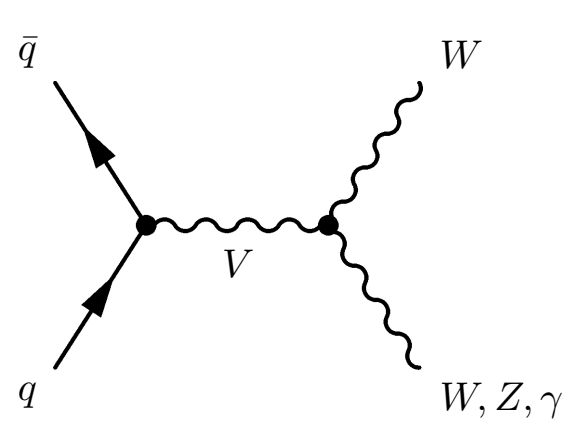

(a)

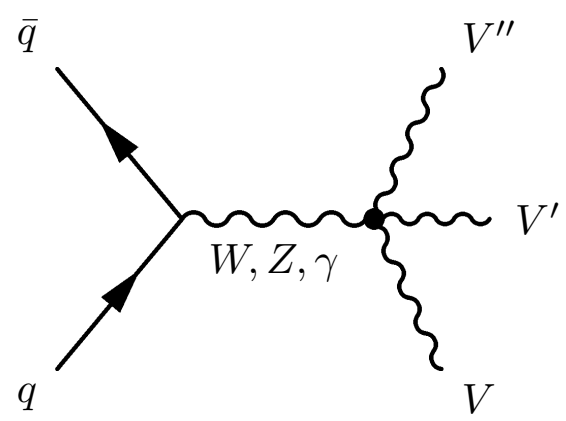

(c)

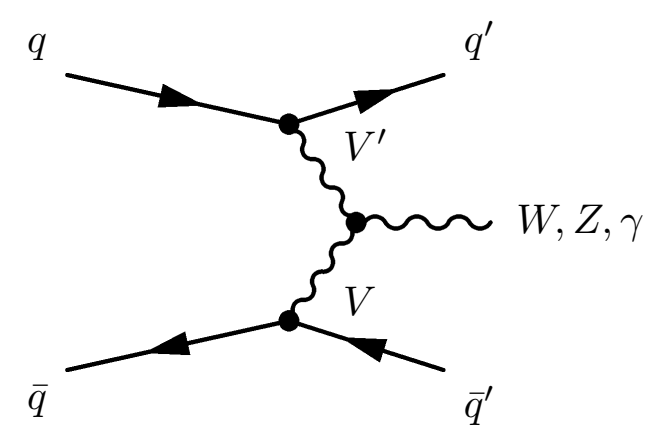

(b)

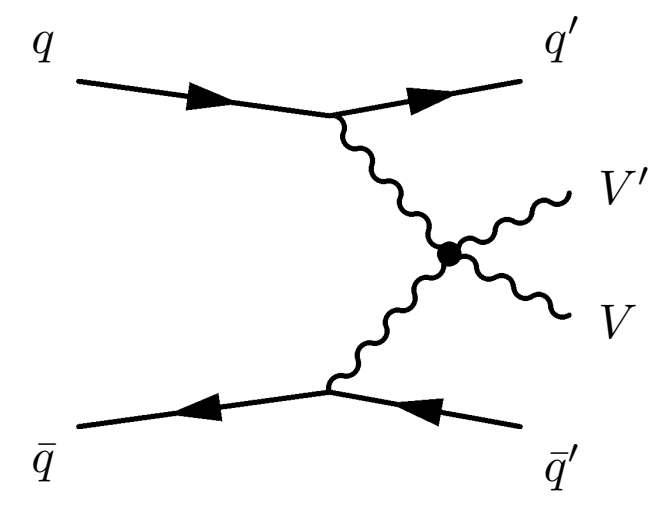

(d)

Figure 1: Prominent examples of tree level Feynman diagrams involving vertices with three or four vector gauge bosons in $p p$ collisions, namely (a) s-channel di-boson production, (b) vector boson fusion, (c) s-channel tri-boson production, and (d) vector boson scattering.

(EFT). The standard model $S U_{C}(3) \otimes S U_{L}(2) \otimes U_{Y}(1)$ symmetry $[17,18,19]$ has operators of up to mass dimension 4. If new physics exists an expansion with higher dimensional operators at a heavier mass scale $\lambda$ suggests itself. The EFT expands the SM Lagrangian with additional terms describing the contributions of new physics with an operator product expansion:

$$
\mathcal{L}=\mathcal{L}_{S M}+\sum_{i} \frac{c_{i}^{(6)}}{\Lambda^{2}} \mathcal{O}_{i}^{(6)}+\sum_{j} \frac{c_{j}^{(8)}}{\Lambda^{4}} \mathcal{O}_{j}^{(8)}+\cdots
$$

where $c_{i}$ are dimensionless Wilson coefficients, $\Lambda$ is a heavier energy scale at which the new physics might be occurring, and $\mathcal{O}_{i}$ are operators constructed from SM fields. Operators of dimension 5 violate lepton number conservation [20], so assuming baryon and lepton number conservation the lowest order operator is of dimension 6 .

Historically, experiments at LEP have set limits on triple gauge coupling vertices in the formulation of anomalous couplings of gauge vector boson $[21,22,23,24]$ motivated to verify the $\mathrm{C}, \mathrm{P}$ and $\mathrm{T}$ invariance and magnetic dipole and electric quadrupole moment of the $W^{ \pm}$of the tri-boson vertex at a time where neither the Higgs, not the $W^{ \pm}$and $Z$ boson were directly observed. Equation 4 explicits the TGC Lagrangian from [13] with $\tilde{V}_{\mu \nu}=\frac{1}{2} \epsilon_{\mu \nu \rho \sigma} V^{\rho \sigma}$. 


$$
\begin{aligned}
\mathcal{L}_{T G C}= & i g_{W W V}\left[g_{1}^{V}\left(W_{\mu \nu}^{\dagger} W^{\mu} V^{\nu}-W_{\mu}^{\dagger} V_{\nu} W^{\mu \nu}\right)+\kappa_{V} W_{\mu}^{\dagger} W_{\nu} V^{\mu \nu}+\frac{\lambda_{V}}{m_{W}^{2}} W_{\mu}^{\dagger \nu} W_{\nu}^{\rho} V_{\rho}^{\mu}\right. \\
& +i g_{4}^{V} W_{\mu}^{\dagger} W_{\nu}\left(\partial^{\mu} V^{\nu}+\partial^{\nu} V^{\mu}\right)-i g_{5}^{V} \epsilon^{\mu \nu \rho \sigma}\left(W_{\mu}^{\dagger} \delta_{\rho} W_{\nu}-\delta_{\rho} W_{\mu}^{\dagger} W_{\nu}\right) V_{\sigma} \\
& \left.+\tilde{\kappa}_{V} W_{\mu}^{\dagger} W_{\nu} \tilde{V}^{\mu \nu}+\frac{\tilde{\lambda}_{V}}{m_{W}^{2}} W_{\mu}^{\dagger \nu} W_{\nu}^{\rho} \tilde{V}_{\rho}^{\mu}\right]
\end{aligned}
$$

The Lagrangian is constructed from five dimension four operators and two dimension six operators (coupling parameters $\lambda$ and $\tilde{\lambda}$ ). The seven operators in equation 4 represent the most general extension of the SM tri-boson Lagrangian, containing all possible Lorentz structures with the lowest dimensional operators possible. Higher dimensional operators can be constructed by replacing $V^{\mu}$ with $\delta^{2 n} V^{\mu}$ with $n$ a positive integer [13] suppressed by additional $\frac{1}{m_{W}^{2 n}}$ mass terms to keep the coupling dimensionless. The couplings $g_{1}^{V}, \kappa_{V}$ and $\lambda_{V}$ conserve $C$ and $P$ symmetries separately, while the other couplings violate $C$ or $P$ or both symmetries.

Similarly, neutral triple gauge couplings that are forbidden in the SM were constructed in an effective Lagrangian approach $[25,26]$ with two classes of Lorentz invariant operators. The first class describe a $Z \gamma$ final state with a virtual $Z^{*}$ or $\gamma^{*}$ as propagator in the $s$-channel, with eight couplings $h_{i=1,2,3,4}^{V=Z, \gamma}$ describing the anomalous contribution. The second class has a $Z Z$ final state again with a virtual $Z^{*}$ or $\gamma^{*}$ as propagator in the $s$-channel. Here we have four couplings defined, $f_{i=4,5}^{V=W, Z}$. The couplings $h_{i=1,2}^{V=Z, \gamma}, f_{4}^{V=Z, \gamma}$ are $C P$-odd whereas $h_{i=3,4}^{V=Z, \gamma}, f_{5}^{V=Z, \gamma}$ are $C P$-even. For neutral couplings $h_{i=1,3}^{V=Z, \gamma}, f_{i=4,5}^{V=Z, \gamma}$ and $h_{i=2,4}^{V=Z, \gamma}$ the lowest dimension is six or eight, respectively.

Generally, the extended Lagrangians do not conserve unitarity at high energies due to the increase of the scattering amplitude with energy. To cure the problem the use of an ad-hoc form-factor was introduced [27], and the unitarity limits, i.e. the maximum allowed energy scale at a given coupling value were calculated $[28,29]$. While the unitarity limits were comfortably high for the experiments operating at the LEP and Tevatron colliders, the LHC started to charter unitarity violating territory. The function of form factors is to dampen the contribution of the additional operators with increasing $\hat{s}$. Typically, a dipole form-factor as proposed in [27] and motivated by the nucleon form factors have been employed to guard unitarity of the cross section,

$$
c(\hat{s})=\frac{c_{0}}{\left(1+\hat{s} / \Lambda^{2}\right)^{n}}
$$

, with $\Lambda^{2}$ the scale of a new interaction that causes the anomalous coupling $c$ to deviate from zero and $n$ suitably chosen to control the rise of the operator contribution associated to $c_{0}$ with $\hat{s}$. The price to pay is the loss of generality of the description of new physics as an explicit assumption about the high energy behaviour has to be made. While ad-hoc form-factor was commonly used for results from LEP or the Tevatron as it did not influence the sensitivity of the analyses significantly, the ATLAS and CMS experiments at LHC were suffering in terms of sensitivity when using the form-factor unitarisation scheme. Consequently, measurements were presented with and without application of form-factors leading to the situation that the results on limits on anomalous couplings from the two experiments were in some instances difficult to compare, e.g. the early $\sqrt{s}=7 \mathrm{TeV}$ measurement of $W^{+} W^{-}$production from ATLAS [30] measure anomalous TGC couplings with an without form-factors, while the corresponding measurement from CMS [31] does not provide unitarized limits.

Because of these interpretation problems the effective field theory (EFT) framework gained in popularity over anomalous couplings approach, as evidenced by eg. [32] and based on initial work in $[33,24,34,35,36]$. A complete set of dimension six operators for the $W^{ \pm} W^{ \pm} Z$ and $W^{ \pm} W^{ \pm} \gamma$ vertices respecting $S U_{C}(3) \otimes S U_{L}(2) \otimes U_{Y}(1)$ is listed in [37], but only a few are relevant for electroweak di-boson 
production. Only three independent dimension six operators are left contributing to nonstandard triple gauge boson vertices when imposing $C$ and $P$ conservation [34]:

$$
\begin{aligned}
\mathcal{O}_{W W W} & =\operatorname{Tr}\left[\hat{W}_{\mu \nu} \hat{W}^{\nu \rho} \hat{W}_{\rho}^{\mu}\right] \\
\mathcal{O}_{W} & =\left(D_{\mu} \Phi\right)^{\dagger} \hat{W}_{\mu \nu}\left(D_{\nu} \Phi\right) \\
\mathcal{O}_{B} & =\left(D_{\mu} \Phi\right)^{\dagger} \hat{B}_{\mu \nu}\left(D_{\nu} \Phi\right)
\end{aligned}
$$

with $\Phi$ the Higgs doublet field and

$$
D_{\mu}=\delta_{\mu}+\frac{i}{2} g^{\prime} B_{\mu}+i g \frac{\sigma^{\alpha}}{2} W_{\mu}^{\alpha}
$$

the covariant derivative for an isospin doublet with hypercharge $Y=1 / 2$. Notably, the anomalous couplings parameters of eg. the $W^{ \pm} W^{ \pm} Z$ vertex mentioned above can be translated into Wilson coefficients of the EFT framework with the following relation from [34, 32]:

$$
\begin{aligned}
& g_{1}^{Z}=1+C_{W} \frac{m_{Z}^{2}}{2 \Lambda^{2}} \\
& \kappa_{\gamma}=1+\left(c_{W}+c_{B}\right) \frac{m_{W}^{2}}{2 \Lambda^{2}} \\
& \kappa_{Z}=1+\left(c_{W}-c_{B} \tan ^{2} \theta_{W}\right) \frac{m_{W}^{2}}{2 \Lambda^{2}} \\
& \lambda_{\gamma}=\lambda_{Z}=c_{W W W} \frac{3 g^{2} m_{W}^{2}}{2 \Lambda^{2}} \\
& g_{4}^{V}=g_{5}^{V}=0 \\
& \tilde{\kappa}_{\gamma}=c_{\tilde{W}} \tan ^{2} \theta_{W} \frac{m_{W}^{2}}{2 \Lambda^{2}} \\
& \tilde{\lambda}_{\gamma}=\tilde{\lambda}_{Z}=c_{\tilde{W} W W} \frac{3 g^{2} m_{W}^{2}}{2 \Lambda^{2}}
\end{aligned}
$$

The EFT Ansatz can be translated directly into the anomalous coupling framework only if form-factors are not applied. Contrary to the anomalous coupling framework the EFT is explicitly constructed to describe the effective low energy behaviour of new physics situated at a much higher scale $\Lambda$. The description breaks down once the scale $\Lambda$ is experimentally accessible, and hence the new interaction would be measured directly and no longer is a subtle corrections to the SM Lagrangian. So the responsibility of using the theory sensibly is with the experimentalist to judge when the EFT ansatz is appropriate. For a thorough discussion of EFT and unitarity the reader is referred to [35]. The experiments at the LHC use for their latest measurements of di-boson production the EFT formalism in addition to the anomalous coupling formalism.

With the change from discovery of the Higgs boson to precision measurements of its properties, a generalised EFT approach including scalar Higgs fields and electroweak vector bosons fields was needed, extending the operator base to cover multi-boson production vertices with scalar and vector bosons, and consequently set limits or find hints of BSM physics using the entire gauge boson sector. This review will discuss experimental results from multi-vector-boson studies only, however there are ambitions of the LHC experiments to bring together the interpretation frameworks of multi-vector boson studies and Higgs studies to profit from the increased sensitivity. A recent study combining data from LEP and LHC on Higgs, di-boson and electroweak production demonstrates the complementarity of the different data sets when setting limits on EFT extensions of the SM [38]. The EFT can be formulated using different bases, i.e. formulations of operators to cover all possible gauge invariant interaction of SM fields which are complete and non-redundant. Because of the requirement of completeness the physical 


\begin{tabular}{|c|c|c|c|c|c|}
\hline \multicolumn{2}{|r|}{$X^{3}$} & \multicolumn{2}{|r|}{$\varphi^{6}$ and $\varphi^{4} D^{2}$} & \multicolumn{2}{|r|}{$\psi^{2} \varphi^{3}$} \\
\hline$Q_{G}$ & $f^{A B C} G_{\mu}^{A \nu} G_{\nu}^{B \rho} G_{\rho}^{C \mu}$ & $Q_{\varphi}$ & $\left(\varphi^{\dagger} \varphi\right)^{3}$ & $Q_{e \varphi}$ & $\left(\varphi^{\dagger} \varphi\right)\left(\bar{l}_{p} e_{r} \varphi\right)$ \\
\hline$Q_{\widetilde{G}}$ & $f^{A B C} \widetilde{G}_{\mu}^{A \nu} G_{\nu}^{B \rho} G_{\rho}^{C \mu}$ & $Q_{\varphi \square}$ & $\left(\varphi^{\dagger} \varphi\right) \square\left(\varphi^{\dagger} \varphi\right)$ & $Q_{u \varphi}$ & $\left(\varphi^{\dagger} \varphi\right)\left(\bar{q}_{p} u_{r} \widetilde{\varphi}\right)$ \\
\hline$Q_{W}$ & $\varepsilon^{I J K} W_{\mu}^{I \nu} W_{\nu}^{J \rho} W_{\rho}^{K \mu}$ & $Q_{\varphi D}$ & $\left(\varphi^{\dagger} D^{\mu} \varphi\right)^{\star}\left(\varphi^{\dagger} D_{\mu} \varphi\right)$ & $Q_{d \varphi}$ & $\left(\varphi^{\dagger} \varphi\right)\left(\bar{q}_{p} d_{r} \varphi\right)$ \\
\hline$Q_{\widetilde{W}}$ & $\varepsilon^{I J K} \widetilde{W}_{\mu}^{I \nu} W_{\nu}^{J \rho} W_{\rho}^{K \mu}$ & & & & \\
\hline \multicolumn{2}{|r|}{$X^{2} \varphi^{2}$} & \multicolumn{2}{|r|}{$\psi^{2} X \varphi$} & \multicolumn{2}{|r|}{$\psi^{2} \varphi^{2} D$} \\
\hline$Q_{\varphi G}$ & $\varphi^{\dagger} \varphi G_{\mu \nu}^{A} G^{A \mu \nu}$ & $Q_{e W}$ & $\left(\bar{l}_{p} \sigma^{\mu \nu} e_{r}\right) \tau^{I} \varphi W_{\mu \nu}^{I}$ & $Q_{\varphi l}^{(1)}$ & $\left(\varphi^{\dagger} i \stackrel{\leftrightarrow}{D}_{\mu} \varphi\right)\left(\bar{l}_{p} \gamma^{\mu} l_{r}\right)$ \\
\hline$Q_{\varphi \widetilde{G}}$ & $\varphi^{\dagger} \varphi \widetilde{G}_{\mu \nu}^{A} G^{A \mu \nu}$ & $Q_{e B}$ & $\left(\bar{l}_{p} \sigma^{\mu \nu} e_{r}\right) \varphi B_{\mu \nu}$ & $Q_{\varphi l}^{(3)}$ & $\left(\varphi^{\dagger} i \stackrel{\leftrightarrow}{D} \stackrel{\leftrightarrow}{I}^{I} \varphi\right)\left(\bar{l}_{p} \tau^{I} \gamma^{\mu} l_{r}\right)$ \\
\hline$Q_{\varphi W}$ & $\varphi^{\dagger} \varphi W_{\mu \nu}^{I} W^{I \mu \nu}$ & $Q_{u G}$ & $\left(\bar{q}_{p} \sigma^{\mu \nu} T^{A} u_{r}\right) \widetilde{\varphi} G_{\mu \nu}^{A}$ & $Q_{\varphi e}$ & $\left(\varphi^{\dagger} i \stackrel{\leftrightarrow}{D}_{\mu} \varphi\right)\left(\bar{e}_{p} \gamma^{\mu} e_{r}\right)$ \\
\hline$Q_{\varphi \widetilde{W}}$ & $\varphi^{\dagger} \varphi \widetilde{W}_{\mu \nu}^{I} W^{I \mu \nu}$ & $Q_{u W}$ & $\left(\bar{q}_{p} \sigma^{\mu \nu} u_{r}\right) \tau^{I} \widetilde{\varphi} W_{\mu \nu}^{I}$ & $Q_{\varphi q}^{(1)}$ & $\left(\varphi^{\dagger} i \stackrel{\leftrightarrow}{D}_{\mu} \varphi\right)\left(\bar{q}_{p} \gamma^{\mu} q_{r}\right)$ \\
\hline$Q_{\varphi B}$ & $\varphi^{\dagger} \varphi B_{\mu \nu} B^{\mu \nu}$ & $Q_{u B}$ & $\left(\bar{q}_{p} \sigma^{\mu \nu} u_{r}\right) \widetilde{\varphi} B_{\mu \nu}$ & $Q_{\varphi q}^{(3)}$ & $\left(\varphi^{\dagger} i \stackrel{D_{\mu}^{I}}{I} \varphi\right)\left(\bar{q}_{p} \tau^{I} \gamma^{\mu} q_{r}\right)$ \\
\hline$Q_{\varphi \widetilde{B}}$ & $\varphi^{\dagger} \varphi \widetilde{B}_{\mu \nu} B^{\mu \nu}$ & $Q_{d G}$ & $\left(\bar{q}_{p} \sigma^{\mu \nu} T^{A} d_{r}\right) \varphi G_{\mu \nu}^{A}$ & $Q_{\varphi u}$ & $\left(\varphi^{\dagger} i \stackrel{\leftrightarrow}{D}_{\mu} \varphi\right)\left(\bar{u}_{p} \gamma^{\mu} u_{r}\right)$ \\
\hline$Q_{\varphi W B}$ & $\varphi^{\dagger} \tau^{I} \varphi W_{\mu \nu}^{I} B^{\mu \nu}$ & $Q_{d W}$ & $\left(\bar{q}_{p} \sigma^{\mu \nu} d_{r}\right) \tau^{I} \varphi W_{\mu \nu}^{I}$ & $Q_{\varphi d}$ & $\left(\varphi^{\dagger} i \stackrel{\leftrightarrow}{D}_{\mu} \varphi\right)\left(\bar{d}_{p} \gamma^{\mu} d_{r}\right)$ \\
\hline$Q_{\varphi \widetilde{W} B}$ & $\varphi^{\dagger} \tau^{I} \varphi \widetilde{W}_{\mu \nu}^{I} B^{\mu \nu}$ & $Q_{d B}$ & $\left(\bar{q}_{p} \sigma^{\mu \nu} d_{r}\right) \varphi B_{\mu \nu}$ & $Q_{\varphi u d}$ & $i\left(\widetilde{\varphi}^{\dagger} D_{\mu} \varphi\right)\left(\bar{u}_{p} \gamma^{\mu} d_{r}\right)$ \\
\hline
\end{tabular}

Table 2: Dimension-six operators other than the four-fermion ones as listed in [39].

content of different bases is equivalent. The most commonly used basis is the so called the Warsaw basis [39] as being the first complete and non-redundant set of dimension 6 operator. For the Warsaw basis all operators except for four-fermion interactions are listed in Table 2 as presented in [39]. The operators relevant for triple gauge vector boson interactions are listed under the $X^{3}$ class.

Another popular variant is the SILH basis [40,41], which has been designed to describe the effects of universal theories where new physics couples preferably to the SM bosons. Since both bases are complete it is possible to translate results from one basis to the other [42]. For a comprehensive and more complete discussion on the SM extension with EFT the reader is referred to [43].

\subsection{Calculations}

At the end of run-2 the ATLAS and CMS experiments at the LHC have collected each a data-set corresponding to an integrated luminosity of about $160 \mathrm{fb}^{-1}$ and with these huge data-sets the uncertainties on multi-boson production observables will reach the level of a few percent. Another jump of one order of magnitude in dataset size is expected for the high-luminosity LHC upgrade project. This motivates the development of high-precision calculations from the theory side. For multi-boson production processes at the LHC the production cross section, i.e. the hard partonic scattering part including perturbative corrections, has been calculated for selected processes up the next-to-next-to-leading order (NNLO) in $\alpha_{s}$ QCD and next-to-leading order (NLO) in $\alpha$ electroweak (EW). First basic predictions for multi-boson production were calculated at Born level fixed order, i.e. leading-order (LO), and with undecayed vector bosons in the final state. The LO calculations were improved by adding particle decays, off-shell contributions, and universal corrections [44]. In recent years more sophisticated calculation including higher order correction in QCD and EW, as well as additional hard jets in the final state, became successively available.

Table 3 lists some recent calculations of multi-boson processes, demonstrating that di-boson pro- 
Table 3: Recent calculations of multiboson processes.

\begin{tabular}{c|c|c|c|c}
\hline Process & O(QCD $)$ & O(EWK) & Comment & Reference \\
\hline$W^{+} W^{-}$ & NNLO & LO & on-shell & {$[45]$} \\
$W^{+} W^{-}$ & NNLO & LO & off-shell with spin correlations & {$[46]$} \\
$W^{+} W^{-}$ & LO & NLO & off-shell & {$[47,48]$} \\
$Z Z$ & NNLO & LO & on-shell & {$[49]$} \\
$Z Z$ & NNLO & LO & off-shell with spin correlations & {$[49,50]$} \\
$Z Z$ & LO & NLO & off-shell & {$[51]$} \\
$W^{ \pm} Z$ & NNO & LO & off-shell with spin correlations & {$[52]$} \\
$W^{ \pm} Z$ & LO & NLO & off-shell & {$[53]$} \\
$g g \rightarrow W^{+} W^{-}$ & NLO & LO & gluon fusion process & {$[54]$} \\
$g g \rightarrow Z Z$ & NLO & LO & gluon fusion process & {$[55]$} \\
VBS $W^{ \pm} W^{ \pm}$ & NLO & NLO & off-shell with spin correlations & {$[56]$} \\
$W^{ \pm} W^{ \pm} W^{\mp}$ & LO & NLO & off-shell & {$[57]$} \\
$W^{ \pm} W^{ \pm} W^{\mp}$ & NLO & LO & off-shell with spin correlations & {$[58]$} \\
$\gamma \gamma V$ & NLO & NLO & off-shell with spin correlations & {$[59]$} \\
$W^{ \pm} Z Z$ & NLO & NLO & off-shell with spin correlations & {$[60]$} \\
$Z Z Z$ & NLO & NLO & off-shell with spin correlations & {$[61]$}
\end{tabular}

cesses are now known to NNLO precision in $\alpha_{s}$ and NLO in $\alpha$, whereas tri-boson processes have been calculated to NLO in $\alpha_{s}$ and $\alpha$.

Calculation at higher order involves additional diagrams to the Born level diagrams contributing to the production cross section. These diagrams contain either virtual particles, also called virtual corrections, or lead to additional particles in the final state, so called real corrections. Example Feynman diagrams for both types of corrections are shown in Figure 2.

The higher order corrections can have a significant impact on production cross section and kinematic observables. Typically, the cross section is increasing with higher order corrections in $\alpha_{s}$, the real gluon emission produce additional jets in the event, and the kinematics at high $p_{\mathrm{T}}$ are enhanced. Higher order corrections in $\alpha$ tend to have the opposite effect and typically suppress the high $p_{\mathrm{T}}$ regions. The order of magnitude for NNLO QCD and NLO EWK corrections is roughly comparable as $\alpha_{s}^{2} \approx \alpha$ at the EWK scale. An example of NLO and NNLO effects on the inclusive $p_{\mathrm{T}}$ spectrum of the leading $W^{ \pm}$and the invariant mass of the di-boson system in $W^{+} W^{-}$production is shown in Figure 3 from [46]. Compared

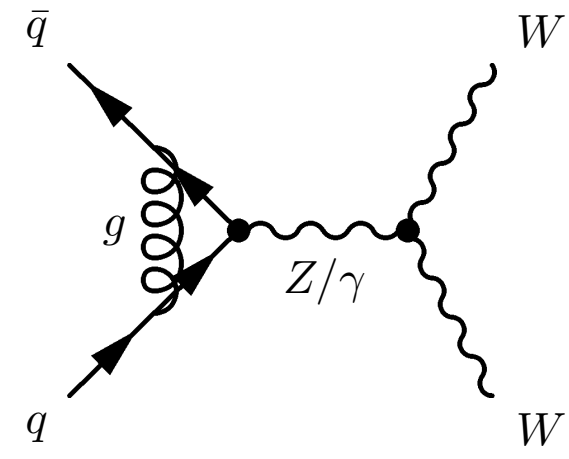

(a)

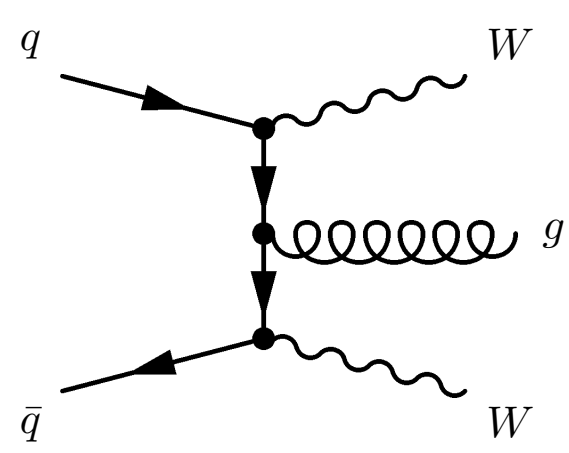

(b)

Figure 2: Examples of NLO Feynman diagrams for $W^{+} W^{-}$production in $p p$ collisions, namely (a) virtual one-loop gluon contribution, (b) real emission of a gluon. 


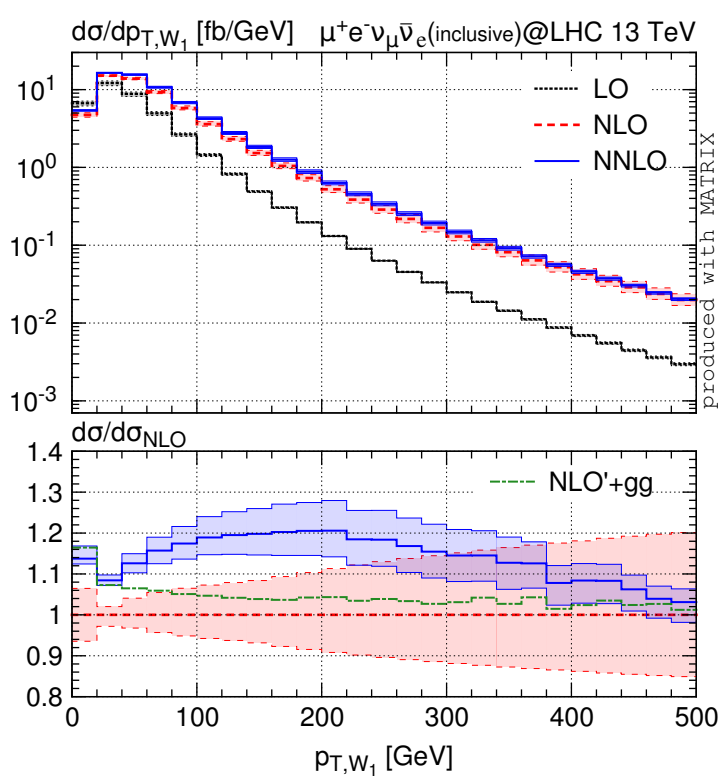

(a)

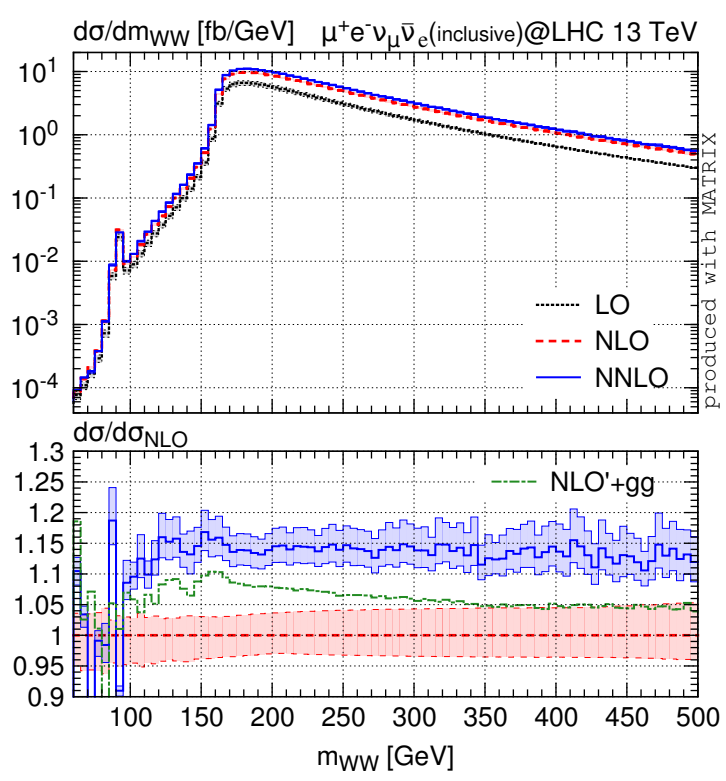

(b)

Figure 3: Distribution of the leading $W^{ \pm} p_{\mathrm{T}}(\mathrm{a})$ and the invariant mass of the di-boson system (b) in $W^{+} W^{-}$production calculated at LO (black, dotted), NLO (red, dashed), and NNLO (blue, solid) at $\sqrt{s}=13 \mathrm{TeV}$ from [46]. The lower ratio plot shows the NLO'+gg (green, dotdashed) and NNLO predictions over NLO prediction, the band indicates the scale dependence of the NLO and NNLO prediction.

to the LO calculation the inclusive production cross section increases by $65 \%$ for the NLO calculation (taking into account the contribution from gluon fusion which is implicit in the NNLO calculation), and $75 \%$ for the NNLO calculation.

The real emission of gluons from the incoming quarks causes additional gluon jets in the event. Looking at experimental cuts applied by the experiments in a typical $W^{+} W^{-}$analysis a jet veto is applied to suppress important background contributions from top pair production. Applying typical kinematic selection and acceptance cuts used by the LHC experiments to mimic the experimentally observable phase space, the predictions are quite different from the inclusive results. As an example the $p_{\mathrm{T}}$ spectrum of the leading lepton and the invariant mass of the dilepton system in $W^{+} W^{-}$production is shown in Figure 4, also from [46], with these cuts applied. The NNLO calculation shows a clear enhancement in the high $p_{\mathrm{T}}$ region with respect to the NLO calculation (a) and in the invariant di-lepton mass spectrum of up to $40 \%$, however the LO calculation has surprisingly the hardest $p_{\mathrm{T}}$ spectrum, mainly due to the application of a jet-veto in the selection. These regions of phase space are also the most sensitive to EFT interpretations of physics beyond the SM; the understanding of higher order corrections is thus very important for the estimation of BSM effects. With respect to the LO calculation the fiducial cross section increases by $20 \%$ for NLO and by $17 \%$ for the NNLO calculation.

The inclusion of NLO electroweak corrections has typically the opposite effect of NLO QCD correction, i.e. the production cross section is decreased with respect to LO and the $p_{\mathrm{T}}$ spectrum is softened. The additional $q \gamma$ production channel counteracts the decrease of the EW NLO correction but is generally smaller then the $q \bar{q}$ induced negative correction originating from electroweak Sudakov logarithms. A calculation of NLO EW correction for the $W^{ \pm} Z$ production process compared to LO illustrates this behaviour, as shown in Figure 5. The overall NLO EW correction to the fiducial cross section in this set-up amounts to about $-4 \%$, and is found less pronounced in the inclusive case $(-1 \%)$. As in the case of higher order QCD corrections the NLO EW correction is enhanced in the high $p_{\mathrm{T}}$ and large $\hat{s}$ 


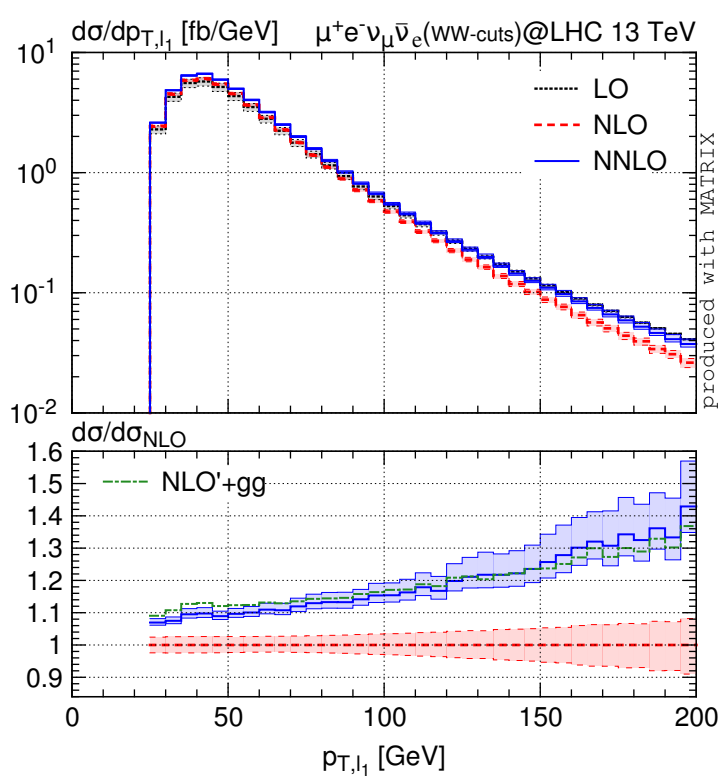

(a)

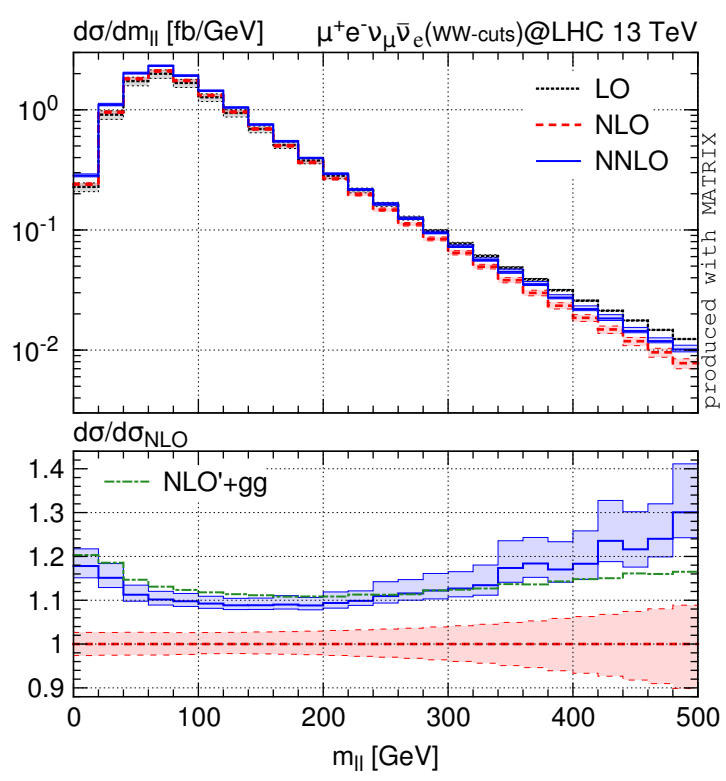

(b)

Figure 4: Distribution of the leading lepton (a) and the invariant mass of the di-lepton system (b) in $W^{+} W^{-}$production calculated at LO (black, dotted), NLO (red, dashed), and NNLO (blue, solid) at $\sqrt{s}=13 \mathrm{TeV}$. The lower ratio plot shows the NLO'+gg (green, dot-dashed) and NNLO in $\alpha_{s}$ predictions over NLO prediction, the band indicates the scale dependence of the NLO and NNLO prediction.

regions of the phase space, but with a negative sign.

At the event generator level, progress has been achieved to make QCD NLO precision available. Event generators include the simulation of the non-perturbative hadronization phase of final state partons, so called "parton-shower". The parton-showers have to be matched to the perturbative calculation and different matching schemes have been developed, e.g. the POWHEG method [63, 64] or MC@NLO [65, 66], with the purpose to maintain the NLO in QCD accuracy of the matrix element calculation as the full logarithmic accuracy of the parton shower resummation. NNLO QCD calculations have been matched to parton showers for e.g. associated Higgs production [67] and Drell-Yan [68] processes, and recently also for di-boson processes [69]. Various event generators are being used for multi-boson final-states, capable of $(\mathrm{N}) \mathrm{NLO}$ in QCD parton shower matching, and in some cases featuring the implementation of additional EFT operators and anomalous gauge couplings, e.g. MadGraph5_aMCONLO [70] (automated NLO QCD, EFT, and also automated NLO EWK [71]), POWHEGBOX [72, 73, 74, 75, 76] (EFT, aGC, NNLO QCD [69]), Sherpa [77](automated NLO QCD and EWK, EFT), VBFNLO [78] (aGC), to name a popular few.

\section{Experimental Methods}

\subsection{Detector Characteristics}

The two multipurpose experiments at the LHC are ATLAS [79] and CMS [80]. The experiments are designed to provide hermetic coverage to detect muons, electrons and hadronic particles with precision tracking and calorimetric information. The hermetic coverage allows to infer non-interacting neutrinos by the determination of missing transverse momentum. The biggest component is the magnet system to provide the bending power for charged particle momentum determination. ATLAS was designed 


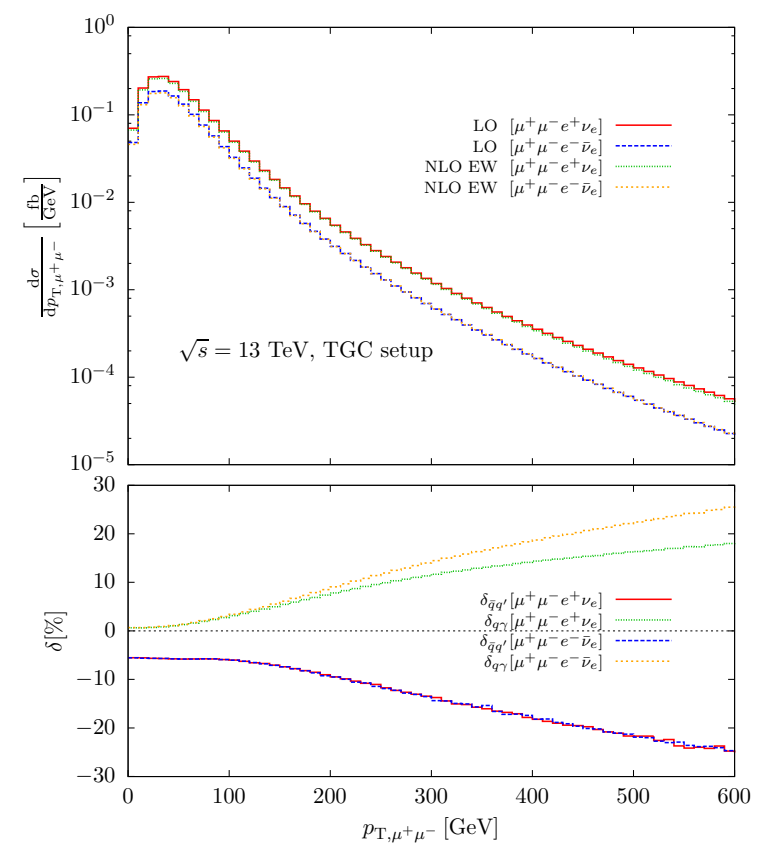

(a)

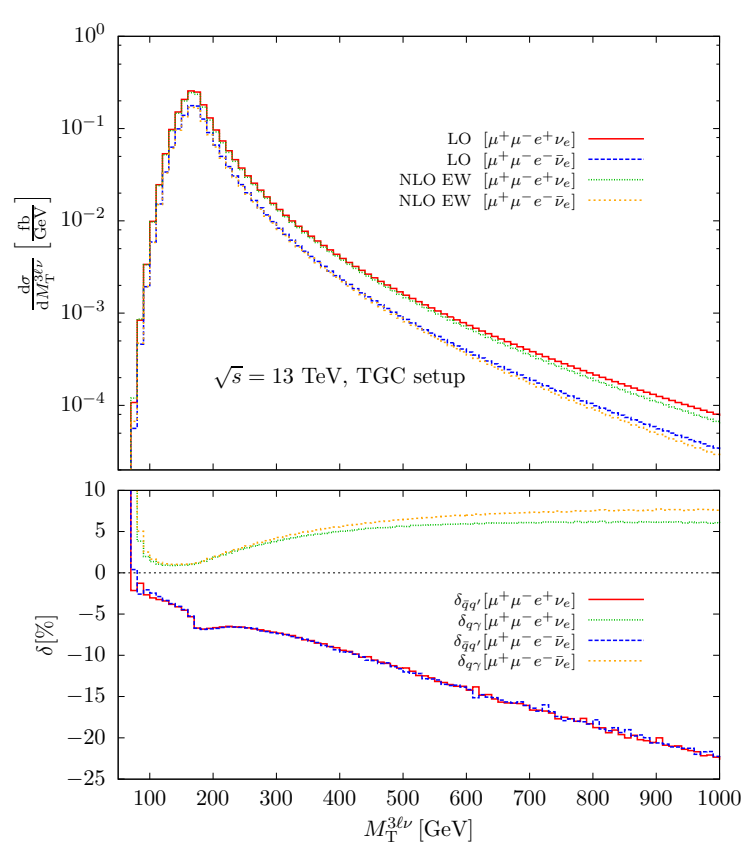

(b)

Figure 5: Distribution of the $p_{\mathrm{T}}$ of the $\mathrm{Z}$ candidate (a) and the transverse mass of the 4 lepton system (b) for $W^{ \pm} Z$ production at $\sqrt{s}=13 \mathrm{TeV}$ comparing the LO to the NLO EW calculation from [53]. Similar acceptance cuts as defined in [62] to define the fiducial volume have been applied. The lower ratio plot indicates the relative contribution of the quark-quark $\left(\bar{q} q^{\prime}\right)$ and quark-photon $(q \gamma)$ processes.

for the ability to precisely reconstruct muons with the muon spectrometer alone and the necessary bending power is realised with an air-coil toroidal outer magnet system, while a solenoid in front of the electromagnetic (EM) calorimeter provides the bending power for the inner tracking systems. ATLAS muon momentum measurements are thus not relying on the inner tracking detector systems, but the addition of the inner tracking systems improves the muon momentum resolution in the transverse momentum region up to about $200 \mathrm{GeV}$ due to the dominance of the multiple scattering term and the fluctuations in the energy loss in the calorimeters in the resolution uncertainty. CMS opted for compact single solenoid design providing the bending power for the inner detector system and containing the calorimeter, while the iron return yoke contains the muon spectrometer. The CMS muon system is capable of providing a standalone muon measurement but the best transverse momentum resolution is achieved in combination with the inner silicon tracking detector up to transverse momenta of $2 \mathrm{TeV}$.

The inner tracking systems of ATLAS and CMS are designed to provide precision measurements of the transverse momenta of charged particles down to about $p_{\mathrm{T}}$ of $\mathcal{O}(100) \mathrm{MeV}$ and $|\eta|<2.5$ in pseudorapidity. In terms of resolution CMS profits from the twice as large magnetic field of the central solenoid and has a significantly better track $p_{\mathrm{T}}$ resolution compared to ATLAS. Both experiments use segmented silicon pixel and strip detectors in the inner layers of the inner tracking system, but differ in the choice of technology in the outer tracker part. CMS is using an all Silicon design up the outer radius $R=1.1 \mathrm{~m}$, while ATLAS opted for a Transition Radiation Tracker (TRT) with $0.5 \mathrm{~m}<R<1.1 \mathrm{~m}$ and particle identification capability to distinguish electron and pions. Despite the differences in technology the overall performance of the tracking systems are similar.

Both ATLAS and CMS feature hermetic calorimetry for hadrons and electrons/photons, but differ in the placement of the calorimeter. ATLAS has chosen to position the LAr hadronic and EM calorimeter outside the central solenoid. This allows to provide sufficient radiation lengths to contain 
the longitudinal shower development, but the resolution for electromagnetic showers is limited by the significant energy loss in the solenoid just in front of the barrel calorimeter. In the case of CMS the entire EM and most of the hadronic calorimetry is placed inside the solenoid, resulting in superior EM resolution compared to ATLAS but less radiation length for hadronic objects are available. This is partially mitigated by the installation of a hadronic tail catcher outside the solenoid coil, but overall the resolution is worse compared to ATLAS. The key performance figures of both experiments [81] are summarised in Table 4.

\subsection{Event reconstruction and Observables}

For precision studies of multi-boson final states the leptonic decay channels are of primary importance due to the cleanliness of the leptonic signal. The semi-leptonic and hadronic final states become interesting in statistically limited final states with three bosons or to enhance the sensitivity to anomalous couplings in di-boson final states. Jets and specifically forward jets are integral part of signatures for vector boson scattering processes. The reconstruction of photons, leptons, jets and missing transverse energy utilises the different detector components (tracking, calorimetry, muon detection), and the precision of the candidates kinematic parameters is determined by the detector performance.

Electron candidates are formed from clusters in the EM calorimeters, that are associated to a track from the inner detector systems, using shower shape information and additionally particle identification information of the TRT in the case of ATLAS [82]. Hadronic jets are a significant source of misidentified or non-prompt electrons that can be effectively rejected using isolation requirements. Isolation criteria are based on the scalar sum of calorimetric energy deposits in the annulus of the electron candidate with a radius of $\Delta R=\sqrt{\eta^{2}+\phi^{2}}$ of 0.3 or 0.4 , or the sum of charged particle tracks around electron candidate within $\Delta R$. Typically, the ratio of the transverse sum to the candidates transverse energy or momentum

is used as isolation cut, $R=\frac{p_{T}}{\sum_{i} p_{T, i}}$. Besides these tracking and calorimetry based isolation variables, CMS also opted for a combination of tracks and calorimetric cluster with the particle flow technique to further enhance the discrimination power [83]. The sign of the charge is induced from the curvature of the associated track. The main contribution to charge misidentified electron candidates stems from Bremsstrahlung of electrons, with subsequent electron-positron pair conversion and assignment of the "wrong" track to the initial electrons. The fraction of charge misidentified electrons increases with $p_{\mathrm{T}}$ and $\eta$ and typically reaches, depending on the working point of the reconstruction algorithms, levels of $\mathcal{O}(1 \%)$. Photons are identified from the cluster shape information of the electromagnetic calorimeter and additionally isolation requirements are applied. Photon conversions to electron-positron pairs are identified with additional tracking information.

The muon reconstruction starts with tracks in the muon chambers, which allows to identify candidates with reasonable transverse momentum resolution. The muon detector tracks are matched to inner detector tracks to further improve the resolution, and isolation criteria are applied to reject muons from non-prompt production processes, eg. heavy quark decays. Both experiments have also algorithms to start with inner detector tracks and find matching muon hits to complement the "outside-in" approach $[84,85]$. The efficiency is comparable for both experiments with reconstruction efficiencies close to $99 \%$ for centrally produced muons. A typical momentum resolution of $1-2 \%$ for CMS and $2-3 \%$ for ATLAS in the intermediate $20 \mathrm{GeV}<p_{\mathrm{T}}<100 \mathrm{GeV}$ range, relevant for vector boson production, is reached.

For the reconstruction of jets ATLAS utilises the lateral and longitudinal calorimetric information to build topological cell clusters [86], that are then clustered to jets with algorithms such as anti-kT [87] for small diameter jets $(0.4<R<1.0)$ and Cambridge Aachen algorithm [88] for larger jets $(R=1.2)$. The approach of looking only at calorimeter cells information can be enhanced with adding tracking information to more precisely measure the energy contributed by charged hadrons and electrons. These so called "particle flow jets" have been chosen by CMS as default since 2010 and significantly improve 
Table 4: Basic key figures of the ATLAS and CMS experiments in comparison, adopted from [81]. Most performance data has been obtained from early running and test-beam campaigns. The EM and hadronic resolution has been determined with pion test-beams. The muon resolution is compared for combined reconstruction, i.e. using tracking information in addition to muon spectrometer information.

\begin{tabular}{|c|c|c|c|c|}
\hline Property & \multicolumn{2}{|c|}{ ATLAS } & \multicolumn{2}{|c|}{ CMS } \\
\hline $\begin{array}{l}\text { Dimension } \\
\text { Weight }\end{array}$ & \multicolumn{2}{|c|}{$\begin{array}{c}\text { diameter } 22 \mathrm{~m} \text {, length } 46 \mathrm{~m} \\
7000 \mathrm{t}\end{array}$} & \multicolumn{2}{|c|}{$\begin{array}{c}\text { diameter } 15 \mathrm{~m} \text {, length } 20 \mathrm{~m} \\
12500 \mathrm{t}\end{array}$} \\
\hline $\begin{array}{l}\text { Magnetic Field } \\
\text { (tracking) }\end{array}$ & \multicolumn{2}{|c|}{$\begin{array}{l}2 \mathrm{~T} \text { solenoid and } \\
\text { air coil toroidal magnets }\end{array}$} & \multicolumn{2}{|c|}{$4 \mathrm{~T}$ solenoid } \\
\hline $\begin{array}{l}\text { Technology } \\
B R^{2}\left[\mathrm{~T} \mathrm{~m}^{2}\right] \\
\text { Total Material }\left(X / X_{0}\right) \\
\text { Acceptance }\end{array}$ & \multicolumn{2}{|c|}{$\begin{array}{c}\text { Silicon Pixel and Strip, } \\
\text { Straw tubes TRT } \\
2 \text { to } 2.3 \\
0.3 \text { to } 1.2 \\
|\eta|<2.5\end{array}$} & \multicolumn{2}{|c|}{$\begin{array}{c}\text { Silicon Pixel and Strip } \\
4.6 \text { to } 4.8 \\
0.4 \text { to } 1.5 \\
|\eta|<2.5\end{array}$} \\
\hline \multirow{4}{*}{$\begin{array}{l}\text { EM System } \\
\text { Technology } \\
\text { Granularity } \\
(\Delta \eta \times \Delta \phi) \\
\text { Intrinsic Resolution } \\
\text { Stochastic Term } a \\
\text { Constant Term } b\end{array}$} & Barrel & Endcap & Barrel & Endcap \\
\hline & \multicolumn{2}{|c|}{ Lead/LAr accordion } & \multicolumn{2}{|c|}{$\mathrm{PbWO}_{4}$} \\
\hline & $0.025 \times 0.025$ & $0.025 \times 0.0251$ & $0.017 \times 0.017$ & $\begin{array}{c}0.018 \times 0.003 \text { to } \\
0.088 \times 0.015\end{array}$ \\
\hline & $\begin{array}{l}10 \% \\
0.2 \%\end{array}$ & $\begin{array}{c}10 \text { to } 12 \% \\
0.35 \%\end{array}$ & $\begin{array}{c}3 \% \\
0.5 \%\end{array}$ & $\begin{array}{l}5.5 \% \\
0.5 \%\end{array}$ \\
\hline \multirow{3}{*}{$\begin{array}{l}\text { Hadronic System } \\
\text { Granularity } \\
(\Delta \eta \times \Delta \phi) \\
\text { Intrinsic Resolution } \\
\text { Stochastic Term } a \\
\text { Constant Term } b\end{array}$} & Barrel & Endcap & Barrel & Endcap \\
\hline & $\begin{array}{l}0.1 \times 0.1 \text { to } \\
0.2 \times 0.1\end{array}$ & $\begin{array}{l}0.1 \times 0.1 \text { to } \\
0.2 \times 0.2\end{array}$ & $0.087 \times 0.087$ & $\begin{array}{c}0.087 \times 0.087 \text { to } \\
0.18 \times 0.175\end{array}$ \\
\hline & $\begin{array}{l}45 \% \\
1.3 \%\end{array}$ & $\begin{array}{l}75 \% \\
2.3 \%\end{array}$ & $\begin{array}{l}100 \% \\
7 \%\end{array}$ & \\
\hline Muon System & Barrel & Endcap & Barrel & Endcap \\
\hline \multirow{2}{*}{$\begin{array}{l}\text { Technology }{ }^{1} \\
\text { Acceptance (Trigger) } \\
\text { Combined } \sigma\left(p_{\mathrm{T}}\right) / p_{\mathrm{T}} \\
p=10 \mathrm{GeV}, \eta=0(2) \\
p=100 \mathrm{GeV}, \eta=0(2)\end{array}$} & MDT, RPC & $\begin{array}{l}\text { MDT, TGC, } \\
\text { CSC }\end{array}$ & $\mathrm{DT}, \mathrm{RPC}$ & $\mathrm{CSC}$ \\
\hline & $\begin{array}{l}1.4 \% \\
2.5 \%\end{array}$ & $\begin{array}{l}2.4 \%) \\
2.1 \%)\end{array}$ & \multicolumn{2}{|c|}{$|\eta|<2.4(2.4)$} \\
\hline
\end{tabular}


the jet resolution [89], compensating for the resolution limitations of the hadronic calorimeter. ATLAS also investigated the use of particle flow jets and found an improvement in jets performance [90], however most run-1 and run-2 multiboson analysis use the topological cluster based jets.

For the detection weakly or non-interacting particles, ie. neutrinos, the missing transverse energy $\left(E_{\mathrm{T}}^{\mathrm{miss}}\right)$ forms the detector signature as the initial state of the proton-proton collision has a balanced transverse momentum. The performance of $E_{\mathrm{T}}^{\mathrm{miss}}$ is determined by its constituents, so all objects that are detectable and discussed above, but also by the limited acceptance of the detectors and the additional soft proton-proton collisions producing mostly low $p_{\mathrm{T}}$ particles accompanying the hard scattering event. The resolution of $E_{\mathrm{T}}^{\text {miss }}$ shows a dependence on the number of underlying events due to the increase in soft energy contribution [91, 92], however, in the region relevant to leptonic $W^{ \pm}$decays of around 15 $\mathrm{GeV}$ the dependence has typically a small impact.

For the selection of collision events during data taking for permanent storage the trigger imposes a set of requirements. For leptonic and semi-leptonic decay channels the single and di-lepton triggers are relevant. Typical operating points during run-2 are a minimum $p_{\mathrm{T}}$ threshold of about $27 \mathrm{GeV}$ (single lepton) or $18 \mathrm{GeV}$ (di-lepton) of the lepton $p_{\mathrm{T}}$ with additional requirements on isolation. The exact requirements vary between run-1 and run-2, and depend on the instantaneous luminosity and other run conditions. Fully hadronic final states have to rely on jet and multi-jet triggers, which have a much higher threshold due to high QCD jet production rate compared to leptons, and typical thresholds in run-2 are at or above $400 \mathrm{GeV}$ for single jets. The very high level of contamination from QCD jets and the high trigger thresholds render the fully hadronic decay channel unfeasible for multi-boson analysis focussed on standard model precision measurement, but it remains a sensitive channel for searches of high di-boson mass states and physics beyond the standard model.

\section{Experimental Results}

In this section the main results of the ATLAS and CMS experiments are discussed. The results are grouped in processes (inclusive production, exclusive production) and boson multiplicity (di-boson and tri-boson production).

\subsection{Inclusive di-boson production}

The di-boson process has been well explored at the LHC, since all $V V$ production states with $V=$ $\gamma, W^{ \pm}, Z$ have been observed and production cross section have been measured. The $V V$ process has been observed already before the start of the LHC at LEP $\left(W^{+} W^{-}, Z Z, Z \gamma\right)$ and the Tevatron $\left(W^{+} W^{-}, W^{ \pm} Z, Z Z, W^{ \pm} \gamma, Z \gamma\right)$, and production cross section and limits on anomalous couplings have been determined. A summary of LEP results and combination of anomalous couplings can be found in [93], and similarly for Tevatron results are summarised in [94].

The early measurements of di-boson production at LHC with $\sqrt{s}=7 \mathrm{TeV}$ where merely "rediscoveries" of the various di-boson processes, albeit at a $p p$ collider. With increasing luminosity and $\sqrt{s}$ the experiments at the LHC started to charter new territory in terms of phase space but also precision on anomalous couplings, surpassing LEP and Tevatron limits with run-2 results. In the following the different di-boson processes and the current results of ATLAS and CMS are presented.

\subsubsection{The $V \gamma$ process}

The process of $V \gamma$ with $V=W^{ \pm}, Z$ has a production cross section of $O(10 \mathrm{pb})$, comparable to massive di-boson final states. At LO the process receives contribution from QED radiation of a photon from the incoming virtual quark (initial state radiation) and from radiation from a charged lepton from the 


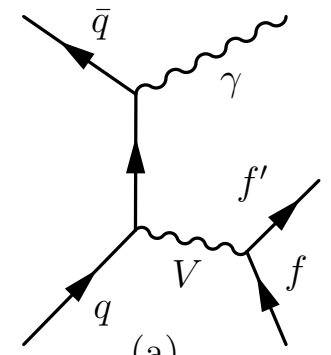

(a)

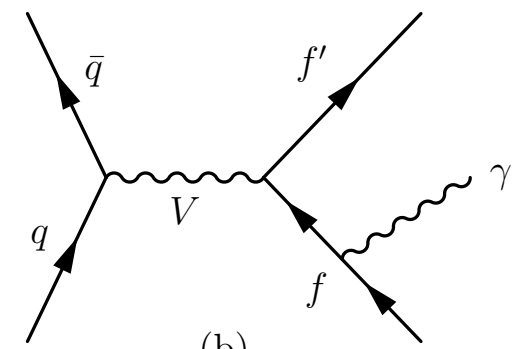

(b)

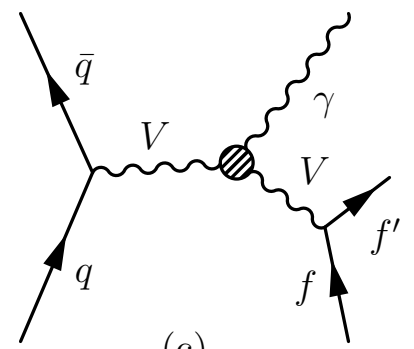

(c)

Figure 6: Feynmandiagrams of LO processes contributing to the $V \gamma$ process [95], with a) initial state radiation, b) final state radiation, and c) triple gauge boson vertex which is only allowed for $V=W^{ \pm}$in the SM.

$V$ decay, and from the TGC vertex in case of $V=W$ (see Figure 6 [95]). In the case of $V=Z$ the TGC vertex is not allowed in the SM.

Previous measurements by experiments at LEP [96] and the Tevatron [97] have already observed the $Z \gamma$ and $V \gamma$ processes, respectively, and set limits on anomalous TGC couplings. However, the increase $\sqrt{s}$ of LHC in comparison, and the large data sample enabled the LHC experiments to set more stringent limits on anomalous TGC couplings. To enhance the electroweak contribution the experiments define fiducial cross sections and compare to calculations. The $W^{ \pm} \gamma$ process has been studied in the electronic and muonic decay channels $W^{ \pm} \gamma \rightarrow \ell^{ \pm} \nu \gamma$. The $Z \gamma$ process offers in addition to its charged leptonic decay modes $Z \gamma \rightarrow \ell^{+} \ell^{-} \gamma$ also the possibility to be studied in $Z \gamma \rightarrow \nu \bar{\nu} \gamma$, and is to neutral anomalous TGC. Absence of final state radiation in $Z \rightarrow \nu \bar{\nu}$ and the higher branching ratio compared to $Z \rightarrow \ell^{+} \ell^{-}$ makes the $\nu \bar{\nu}$ final state more sensitive to anomalous TGC.

Main background sources for the $V \gamma$ signal stem from $W^{ \pm}+$jets and $Z+$ jets production, in which one jet is misidentified as an isolated photon. This background is tackled by data driven techniques, where the probability of a jet faking the signature of an isolated photon is determined from a data-set independent of the signal sample. Other backgrounds are multi-jet events and $t \bar{t}$ pair production in association with a photon, and are generally estimated from simulation. The $Z \gamma \rightarrow \nu \bar{\nu} \gamma$ process also receives substantial background contributions from $W^{ \pm} \gamma$ which is estimated from data with appropriately defined control regions. Signal to background ratio varies typically in the range from about $0.8-$ 1.5 for $W^{ \pm} \gamma \rightarrow \ell^{ \pm} \nu \gamma \quad$ to $3.5-7$ for $Z \rightarrow \ell^{+} \ell^{-}$depending on channel and experiment.

ATLAS and CMS have both published measurements of integrated and fiducial production cross sections of $W^{ \pm} \gamma \rightarrow \ell^{ \pm} \nu \gamma, Z \gamma \rightarrow \ell^{+} \ell^{-} \gamma$ at $\sqrt{s}=7 \mathrm{TeV}$ and $\sqrt{s}=8 \mathrm{TeV}$, and recently ATLAS also published $Z \gamma \rightarrow \nu \bar{\nu} \gamma$ at $\sqrt{s}=13 \mathrm{TeV}$. Fiducial volumes are defined at particle level with kinematic selection criteria on objects and at the event level that are motivated by the experimental acceptance. Hence, ATLAS and CMS differ in the definitions of the fiducial cross sections and so measured and predicted fiducial cross sections cannot be directly compared between different analysis. The crosssections for $Z \gamma \rightarrow \ell^{+} \ell^{-} \gamma$ and $W^{ \pm} \gamma \rightarrow \ell^{ \pm} \nu \gamma$ processes measured at $\sqrt{s}=7,8 \mathrm{TeV}$ are defined as $p_{\mathrm{T}}(\gamma)>15 \mathrm{GeV}, \Delta(\ell, \gamma)>0.7$, and additionally for $Z \gamma$ require $\left.m(\ell \ell)>50(40) \mathrm{GeV}\right)$ for $\mathrm{CMS}$ (ATLAS). ATLAS further requires $|\eta(\ell)|<2.47,|\eta(\gamma)|<2.37$, and $E_{\mathrm{T}}($ jet $)>30 \mathrm{GeV}, \mid \eta($ jet $) \mid<4.4$, $\Delta R($ jet,$x)>0.3$ for jets with $x$ being a lepton, jet, or photon. For the $Z \gamma \rightarrow \nu \bar{\nu} \gamma$ process the cross section is defined as $E_{\mathrm{T}}(\gamma)>145(100,130,150) \mathrm{GeV},|\eta(\gamma)|<1.4(2.37), p_{\mathrm{T}}(\nu \nu)>130(90,100,150)$ for CMS (ATLAS $7 \mathrm{TeV}$ [98], $8 \mathrm{TeV}$ [8]), and $13 \mathrm{TeV}$ [99]). A summary of available measurements of the $V \gamma$ process is given in Table 4.1.1. The measured and predicted exclusive cross sections are in good agreement with each other, while the measured inclusive cross sections seem to be systematically higher than the NLO prediction. This may be attributed to corrections at NNLO in $\alpha_{s}$, as evidenced 
by the ATLAS measurements at $\sqrt{s}=13 \mathrm{TeV}$ which compares to NNLO calculations and finds good agreement for both inclusive and exclusive cross section measurements.

\begin{tabular}{|c|c|c|c|c|c|}
\hline Experiment & process & cross section & $\sqrt{s}[\mathrm{TeV}]$ & measured & reference \\
\hline ATLAS & $W^{ \pm} \gamma \rightarrow \ell^{ \pm} \nu \gamma$ & inclusive & 7 & $2.77 \pm 0.03$ (stat) \pm 0.33 (syst) \pm 0.14 (lumi) pb & {$[100]$} \\
\hline ATLAS & $W^{ \pm} \gamma \rightarrow \ell^{ \pm} \nu \gamma$ & exclusive & 7 & $1.76 \pm 0.03$ (stat) \pm 0.21 (syst) \pm 0.08 (lumi) pb & {$[100]$} \\
\hline ATLAS & $Z \gamma \rightarrow \ell^{+} \ell^{-} \gamma$ & inclusive & 7 & $1.31 \pm 0.02$ (stat) \pm 0.11 (syst) \pm 0.05 (lumi) pb & {$[100]$} \\
\hline ATLAS & $Z \gamma \rightarrow \ell^{+} \ell^{-} \gamma$ & exclusive & 7 & $1.05 \pm 0.02$ (stat) \pm 0.10 (syst) \pm 0.04 (lumi) pb & {$[100]$} \\
\hline ATLAS & $Z \gamma \rightarrow \nu \bar{\nu} \gamma$ & inclusive & 7 & $0.133 \pm 0.013$ (stat) \pm 0.020 (syst) \pm 0.005 (lumi) pb & {$[98]$} \\
\hline ATLAS & $Z \gamma \rightarrow \nu \bar{\nu} \gamma$ & exclusive & 7 & $0.116 \pm 0.010$ (stat) \pm 0.013 (syst) \pm 0.004 (lumi) pb & [98] \\
\hline CMS & $Z \gamma \rightarrow \ell^{+} \ell^{-} \gamma$ & inclusive & 7 & $5.33 \pm 0.08$ (stat) \pm 0.25 (syst) \pm 0.12 (lumi) pb & {$[95]$} \\
\hline CMS & $W^{ \pm} \gamma \rightarrow \ell^{ \pm} \nu \gamma$ & inclusive & 7 & $37.0 \pm 0.8($ stat $) \pm 4.0$ (syst) \pm 0.8 (lumi) pb & [95] \\
\hline CMS & $Z \gamma \rightarrow \nu \bar{\nu} \gamma$ & inclusive & 7 & $21.1 \pm 4.2$ (stat.) \pm 4.3 (syst) \pm 0.5 (lumi) fb & [98] \\
\hline ATLAS & $Z \gamma \rightarrow \ell^{+} \ell^{-} \gamma$ & inclusive & 8 & $1.51 \pm 0.01$ (stat) \pm 0.08 (syst) \pm 0.03 (lumi) pb & {$[8]$} \\
\hline ATLAS & $Z \gamma \rightarrow \ell^{+} \ell^{-} \gamma$ & exclusive & 8 & $1.19 \pm 0.01$ (stat) \pm 0.07 (syst) \pm 0.02 (lumi) pb & {$[8]$} \\
\hline ATLAS & $Z \gamma \rightarrow \nu \bar{\nu} \gamma$ & inclusive & 8 & $68 \pm 4$ (stat) \pm 32 (syst) \pm 1 (lumi) fb & {$[8]$} \\
\hline ATLAS & $Z \gamma \rightarrow \nu \bar{\nu} \gamma$ & exclusive & 8 & $43 \pm 2$ (stat) \pm 10 (syst) \pm 1 (lumi) fb & {$[8]$} \\
\hline CMS & $Z \gamma \rightarrow \ell^{+} \ell^{-} \gamma$ & inclusive & 8 & $2063 \pm 19$ (stat) \pm 98 (syst) \pm 54 (lumi) fb & {$[101]$} \\
\hline CMS & $Z \gamma \rightarrow \ell^{+} \ell^{-} \gamma$ & exclusive & 8 & $1770 \pm 18$ (stat) \pm 115 (syst) \pm 46 (lumi) fb & {$[101]$} \\
\hline CMS & $Z_{\gamma} \rightarrow \nu \bar{\nu} \gamma$ & inclusive & 8 & $52.7 \pm 2.1$ (stat) \pm 6.4 (syst) \pm 1.4 (lumi) fb & {$[102]$} \\
\hline ATLAS & $Z \gamma \rightarrow \nu \bar{\nu} \gamma$ & inclusive & 13 & $83.7 \pm 3.6$ (stat) \pm 6.6 (syst) \pm 1.9 (lumi) fb & {$[99]$} \\
\hline ATLAS & $Z \gamma \rightarrow \nu \bar{\nu} \gamma$ & exclusive & 13 & $52.4 \pm 2.4$ (stat) \pm 3.8 (syst) \pm 1.2 (lumi) fb & [99] \\
\hline
\end{tabular}

Table 5: Summary of measured $V \gamma$ production cross sections from ATLAS and CMS at 7, 8, and 13 $\mathrm{TeV}$ centre-of-mass energies in the $W^{ \pm} \gamma \rightarrow \ell^{ \pm} \nu \gamma, Z \gamma \rightarrow \nu \bar{\nu} \gamma \quad$ and $Z \gamma \rightarrow \ell^{+} \ell^{-} \gamma \quad$ final states. The inclusive cross section is defined for $n_{\text {jet }} \geq 0$ and the exclusive cross section requires $n_{\text {jet }}=0$. The cross section definitions are different for ATLAS and CMS thus the results are not directly comparable. For the $Z \gamma \rightarrow \nu \bar{\nu} \gamma$ process ATLAS uses a different cross section definition between the 7, 8, and $13 \mathrm{TeV}$ measurements.

To extract limits on anomalous TGC parameters the differential spectrum of the transverse momentum of the photon $p_{\mathrm{T}}(\gamma)$ is fitted in the case of CMS, while ATLAS uses a more conservative approach by combining the observed number of exclusive $V \gamma$ candidate events with $E_{\mathrm{T}}(\gamma)>100(600) \mathrm{GeV}$ for the $\sqrt{s}=8(13) \mathrm{TeV}$ analysis. The $Z \gamma \rightarrow \nu \bar{\nu} \gamma$ channel is the most sensitive to the neutral TGC couplings due the favourable branching ratio of $Z$ to neutrinos of $20 \%$.

Figure 7 shows the transverse momentum of the photon at $8 \mathrm{TeV}$ measured by CMS in the $Z \gamma \rightarrow \nu \bar{\nu} \gamma$ channel [102] together with a hypothetical TGC signal, and a comparison of NNLO calculation to measured fiducial cross section at $\sqrt{s}=13 \mathrm{TeV}$ as a function of the jet multiplicity showing very good agreement.

The $95 \%$ CL limits for charged $\left(W^{ \pm} \gamma\right)$ and neutral $(Z \gamma)$ anomalous TGC are summarised in Table 6. For better comparison, only limits obtained without the use of a form factor are listed. ATLAS also provides limits with unitarity-preserving form factors, and in case of the $\sqrt{s}=8 \mathrm{TeV}$ analysis limits as a function of the dipole form factor scale $\Lambda$ [8]. The strong dependence on the partonic centre-of-mass energy, $\hat{s}$, to anomalous TGC explains the by one order of magnitude more stringent limits of the 13 $\mathrm{TeV}$ measurement by ATLAS compared to the run-1 analyses.

\subsubsection{The $W^{+} W^{-}$process, leptonic channel}

The $W^{+} W^{-}$production process is the one with the largest production cross section among the massive vector di-boson processes, but due to presence of two neutrinos in the leptonic final state careful background modelling is essential. Due to its large production cross section the $W^{+} W^{-}$process is an important background process to Higgs production and to searches for new physics in di-boson final states. 


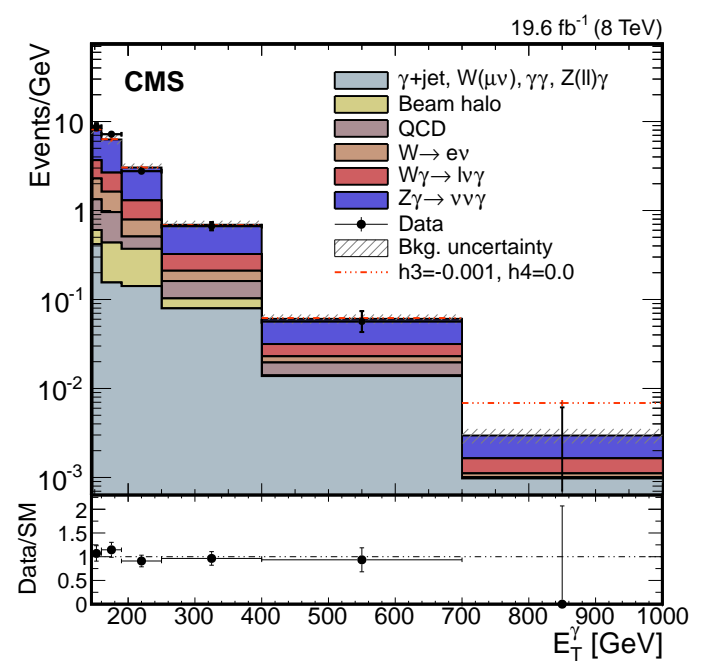

(a)

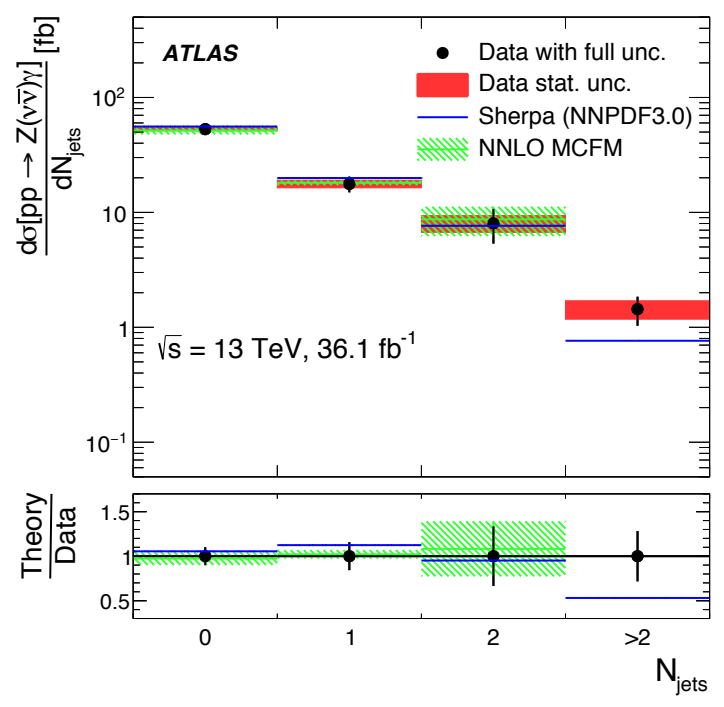

(b)

Figure 7: Figure a) the $E_{\mathrm{T}}(\gamma)$ distribution from CMS [102] in data (points) compared to signal and estimated background contributions (histogram). An anomalous TGC signal with $h_{30}^{V}=-0.001$ is shown (dot-dashed histogram) for comparison. The background uncertainty includes statistical and systematic contributions. Figure b) a comparison of the fiducial measured and predicted cross sections as a function of the number of jets (Njets) compared to different NNLO calculations from ATLAS [99].

\begin{tabular}{|c|c|c|c|c|c|}
\hline & Process & $\sqrt{s}[\mathrm{TeV}]$ & $\mathcal{L}\left[\mathrm{fb}^{-1}\right]$ ATLAS, CMS & $\begin{array}{c}\text { ATLAS } \\
\text { Observed }\end{array}$ & $\begin{array}{c}\text { CMS } \\
\text { Observed }\end{array}$ \\
\hline$\Delta \kappa_{\gamma}$ & $W^{ \pm} \gamma \rightarrow \ell^{ \pm} \nu \gamma$ & 7 & $4.6,5$ & {$[-0.41,0.46]$} & {$[-0.38,0.29]$} \\
\hline$\lambda_{\gamma}$ & $W^{ \pm} \gamma \rightarrow \ell^{ \pm} \nu \gamma$ & 7 & $4.6,5$ & {$[-0.065,0.061]$} & {$[-0.050,0.037]$} \\
\hline$h_{30}^{\gamma}$ & $Z \gamma \rightarrow \ell^{+} \ell^{-} \gamma, Z \gamma \rightarrow \nu \bar{\nu} \gamma$ & 7 & $4.6,5.0$ & {$[-0.015,0.016]$} & $<2.9 \cdot 10^{-3}$ \\
\hline$h_{30}^{Z}$ & $Z \gamma \rightarrow \ell^{+} \ell^{-} \gamma, Z \gamma \rightarrow \nu \bar{\nu} \gamma$ & 7 & $4.6,5.0$ & {$[-0.013,0.014]$} & $<2.7 \cdot 10^{-3}$ \\
\hline$h_{40}^{\gamma}$ & $Z \gamma \rightarrow \ell^{+} \ell^{-} \gamma, Z \gamma \rightarrow \nu \bar{\nu} \gamma$ & 7 & $4.6,5.0$ & {$\left[-9.4 \cdot 10^{-5}, 9.2 \cdot 10^{-5}\right]$} & $<1.5 \cdot 10^{-5}$ \\
\hline$h_{40}^{Z}$ & $Z \gamma \rightarrow \ell^{+} \ell^{-} \gamma, Z \gamma \rightarrow \nu \bar{\nu} \gamma$ & 7 & $4.6,5.0$ & {$\left[-8.7 \cdot 10^{-5}, 8.7 \cdot 10^{-5}\right]$} & $<1.3 \cdot 10^{-5}$ \\
\hline$h_{30}^{\gamma}$ & $Z \gamma \rightarrow \ell^{+} \ell^{-} \gamma, Z \gamma \rightarrow \nu \bar{\nu} \gamma$ & 8 & $20.3,-$ & {$\left[-9.5 \cdot 10^{-4}, 9.9 \cdot 10^{-4}\right]$} & - \\
\hline$h_{30}^{Z}$ & $Z \gamma \rightarrow \ell^{+} \ell^{-} \gamma, Z \gamma \rightarrow \nu \bar{\nu} \gamma$ & 8 & $20.3,-$ & {$\left[-7.8 \cdot 10^{-4}, 8.6 \cdot 10^{-4}\right]$} & - \\
\hline$h_{40}^{\gamma}$ & $Z \gamma \rightarrow \ell^{+} \ell^{-} \gamma, Z \gamma \rightarrow \nu \bar{\nu} \gamma$ & 8 & $20.3,-$ & {$\left[-3.2 \cdot 10^{-6}, 3.2 \cdot 10^{-6}\right]$} & - \\
\hline$h_{40}^{Z}$ & $Z \gamma \rightarrow \ell^{+} \ell^{-} \gamma, Z \gamma \rightarrow \nu \bar{\nu} \gamma$ & 8 & $20.3,-$ & {$\left[-3.0 \cdot 10^{-6}, 2.9 \cdot 10^{-6}\right]$} & - \\
\hline$h_{30}^{\gamma}$ & $Z \gamma \rightarrow \ell^{+} \ell^{-} \gamma$ & 8 &,- 19.5 & - & {$\left[-4.6 \cdot 10^{-3}, 4.6 \cdot 10^{-3}\right]$} \\
\hline$h_{30}^{Z}$ & $Z_{\gamma} \rightarrow \ell^{+} \ell^{-} \gamma$ & 8 &,- 19.5 & - & {$\left[-3.8 \cdot 10^{-3}, 3.7 \cdot 10^{-3}\right]$} \\
\hline$h_{40}^{\gamma}$ & $Z \gamma \rightarrow \ell^{+} \ell^{-} \gamma$ & 8 &,- 19.5 & - & {$\left[-3.6 \cdot 10^{-5}, 3.5 \cdot 10^{-5}\right]$} \\
\hline$h_{40}^{\mathrm{TO}}$ & $Z \gamma \rightarrow \ell^{+} \ell^{-} \gamma$ & 8 &,- 19.5 & - & {$\left[-3.1 \cdot 10^{-5}, 3.0 \cdot 10^{-5}\right]$} \\
\hline$h_{30}^{\gamma}$ & $Z \gamma \rightarrow \nu \bar{\nu} \gamma$ & 8 &,- 19.5 & - & {$\left[-1.1 \cdot 10^{-3}, 0.9 \cdot 10^{-3}\right]$} \\
\hline$h_{30}^{Z}$ & $Z \gamma \rightarrow \nu \bar{\nu} \gamma$ & 8 &,- 19.5 & - & {$\left[-1.5 \cdot 10^{-3}, 1.6 \cdot 10^{-3}\right]$} \\
\hline$h_{40}^{\gamma}$ & $Z \gamma \rightarrow \nu \bar{\nu} \gamma$ & 8 &,- 19.5 & - & {$\left[-3.8 \cdot 10^{-6}, 4.3 \cdot 10^{-6}\right]$} \\
\hline$h_{40}^{Z}$ & $Z \gamma \rightarrow \nu \bar{\nu} \gamma$ & 8 &,- 19.5 & - & {$\left[-3.9 \cdot 10^{-6}, 4.5 \cdot 10^{-6}\right]$} \\
\hline$h_{30}^{{ }^{4}}$ & $Z \gamma \rightarrow \nu \bar{\nu} \gamma$ & 13 & $36.1,-$ & {$\left[-3.7 \cdot 10^{-4}, 3.7 \cdot 10^{-4}\right]$} & {$\left[\begin{array}{cccc}2 & -\end{array}\right]$} \\
\hline$h_{30}^{Z}$ & $Z_{\gamma} \rightarrow \nu \bar{\nu} \gamma$ & 13 & $36.1,-$ & {$\left[-3.2 \cdot 10^{-4}, 3.3 \cdot 10^{-4}\right]$} & - \\
\hline$h_{40}^{\gamma}$ & $Z \gamma \rightarrow \nu \bar{\nu} \gamma$ & 13 & $36.1,-$ & {$\left[-4.4 \cdot 10^{-7}, 4.3 \cdot 10^{-7}\right]$} & - \\
\hline$h_{40}^{\mathrm{HO}}$ & $Z \gamma \rightarrow \nu \bar{\nu} \gamma$ & 13 & $36.1,-$ & {$\left[-4.5 \cdot 10^{-7}, 4.4 \cdot 10^{-7}\right]$} & - \\
\hline
\end{tabular}

Table 6: Observed 95\% CL on $h_{30}^{\gamma}, h_{30}^{Z}, h_{40}^{\gamma}$, and $h_{40}^{Z}$ measured in the $Z \gamma \rightarrow \ell^{+} \ell^{-} \gamma$ and $Z \gamma \rightarrow \nu \bar{\nu} \gamma$ final states from the ATLAS and CMS collaborations. 
ATLAS and CMS observed the $W^{+} W^{-}$production process in the fully leptonic channel and published results for $7 \mathrm{TeV}$ (ATLAS [103], CMS [31]) and $8 \mathrm{TeV}$ (ATLAS [30], CMS [104]) centre-of-mass energy, including the total and differential production cross section, and limits on anomalous TGC and EFT parameters.

Three final states, namely $e e, \mu \mu$, and $e \mu$ are included in the analyses. The contribution from leptonically decaying $\tau$ leptons is included in the signal definition. Although the production cross section is relatively high, the signature of two opposite sign leptons and missing transverse energy is shared with many processes and a careful control of the backgrounds is necessary to achieve a precise measurement. The experiments differ in the definition of the $8 \mathrm{TeV} W^{+} W^{-}$cross section measurement, where CMS does subtract the contribution from the Higgs boson, whereas ATLAS includes the Higgs channel in the signal definition.

Candidate $W^{+} W^{-}$events are selected by requiring two oppositely-charged leptons accompanied with large $E_{\mathrm{T}}^{\text {miss }}$. The dominant background sources are $t \bar{t}$ and single top quark, $W^{ \pm} /+$jets, followed by $Z / \gamma^{*}+$ jets production. In the same flavour channels $e e$ and $\mu \mu$ the substantial background from Drell-Yan lepton pair production is effectively suppressed excluding the invariant mass region around the $Z$ mass and requiring significant $E_{\mathrm{T}}^{\text {miss }}$ in the event. To suppress the $t \bar{t}$ background, events with one or more jets are rejected. Additional requirements on $E_{\mathrm{T}}^{\text {miss }}$ and the use of top quark taggers further reduce the residual background to about $30 \%$. The dominant systematic uncertainty is related to the jet veto efficiency and estimated to be about 3-5\% for the $W^{+} W^{-}$production. The experiments quote a theoretical uncertainty on the signal acceptance due to variations of the parton distribution functions and renormalisation and factorisation scales in the range of $1-2 \%$.

Both ATLAS and CMS provide a measurement of the total cross section for the process $p p \rightarrow W^{+} W^{-}$ and compare to theoretical calculations. At $\sqrt{s}=7 \mathrm{TeV}$ the Higgs process contributes with about $4 \%$ to the total cross section and has not been taken into account in the comparison to the SM predictions. At $\sqrt{s}=8 \mathrm{TeV}$ the contributions is about $8 \%$, is included in the signal definition in the ATLAS measurement, but not in the CMS measurement, hence the slight discrepancy between the two quoted production cross sections.

The total cross section results are summarised in Table 4.1.2. A good agreement between the experiments for the measured cross section as well as for the theoretical predictions is observed. In Figure 8 a) the ATLAS measurement at $\sqrt{s}=8 \mathrm{TeV}$ [103] is compared to NNLO calculation. In Figure $8 \mathrm{~b}$ ) the distribution of the di-lepton invariant mass $m_{l l}$ compared to the signal distribution in the case of additional EFT contributions are shown. The typical enhancement at high $m_{l l}$ for EFT contributions is clearly seen.

\begin{tabular}{|c|c|c|c|c|c|} 
Experiment & cross section & $\sqrt{s}[\mathrm{TeV}]$ & measured [pb] & predicted [pb] & reference \\
\hline ATLAS & total & 7 & $51.9 \pm 2.0$ (stat.) \pm 3.9 (syst.) \pm 2.0 (lumi.) & $44.7_{-1.9}^{+2.1}$ & {$[30]$} \\
ATLAS & total & 8 & $71.1 \pm 1.1$ (stat.) \pm 5.4 (syst.) \pm 1.4 (lumi.) & $63.2_{-1.4}^{+1.6}$ & {$[103]$} \\
CMS & total & 7 & $52.4 \pm 2.0$ (stat.) \pm 4.5 (syst.) \pm 1.2 (lumi.) & $47.0 \pm 2.0$ & {$[31]$} \\
CMS & total & 8 & $60.1 \pm 0.9$ (stat.) \pm 4.5 (syst.) \pm 1.6 (lumi.) & $59.8_{-1.1}^{+1.3}$ & {$[104]$}
\end{tabular}

Table 7: Summary of measured total $W^{+} W^{-}$production cross sections from ATLAS and CMS at 7 and $8 \mathrm{TeV}$ centre-of-mass energies in the $\ell \nu \ell \nu$ final state. The prediction are calculated at NLO (NNLO) in $\alpha_{s}$ for the 7 (8) TeV prediction.

An unfolded spectrum in bins of $p_{\mathrm{T}}$ in the fiducial phase space of the leading lepton is shown in Figure 8, showing good agreement with the SM prediction. The leading lepton $p_{\mathrm{T}}$ and $m_{l l}$ spectra of the $W^{+} W^{-}$process are sensitive to anomalous gauge boson coupling parameters $\Delta \kappa_{\gamma}, \Delta \kappa_{Z}, \lambda_{\gamma}, \lambda_{Z}$, and $\Delta g_{1}^{Z}$ and EFT parameters $c_{B} / \Lambda^{2}, c_{W} / \Lambda^{2}, c_{W W W} / \Lambda^{2}$. In the latest $8 \mathrm{TeV}$ analysis both ATLAS and CMS quote limits for the EFT parameters, which can be converted to anomalous gauge coupling 


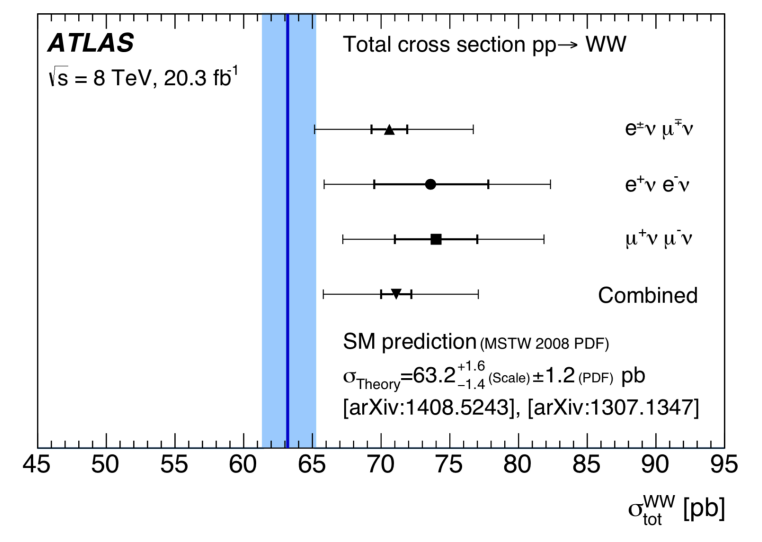

(a)

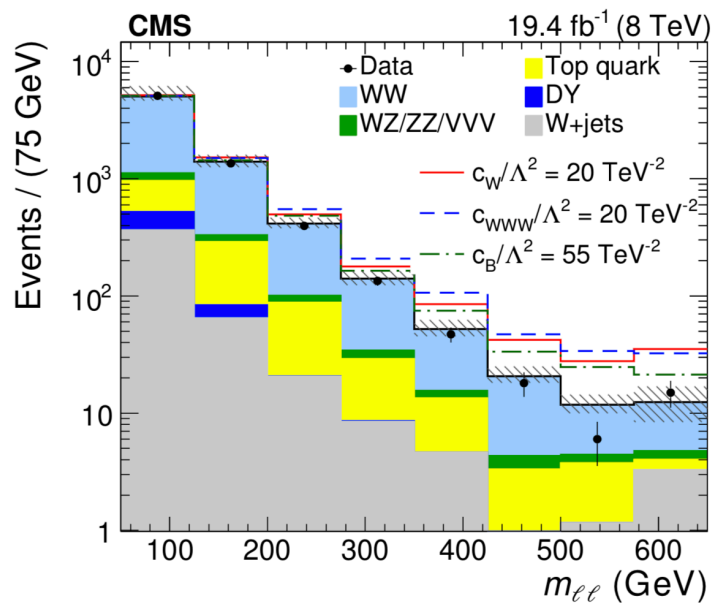

(b)

Figure 8: (a) ATLAS measurement [103] of the production cross section of $W^{+} W^{-}$compared to NNLO calculation at $\sqrt{s}=8 \mathrm{TeV}$. The full uncertainty contains statistical and systematic uncertainties. (b) CMS measurement [104] of the distribution of the di-lepton invariant mass $m_{l l}$ compared to the SM signal and backgrounds requiring no jets in the event. Shown are also the signal distribution in the case of additional EFT contributions. The hatched area on the SM prediction includes the total systematic uncertainty.

\begin{tabular}{ccc}
\hline & Observed (CMS) & Observed (ATLAS) \\
\hline$c_{W W W} / \Lambda^{2}$ & {$[-5.7,5.9]$} & {$[-4.61,4.60]$} \\
$c_{B} / \Lambda^{2}$ & {$[-29.2,23.9]$} & {$[-20.9,26.3]$} \\
$c_{W} / \Lambda^{2}$ & {$[-11.4,5.4]$} & {$[-5.87,10.54]$} \\
\hline
\end{tabular}

Table 8: Observed 95\% CL limits on the EFT parameters $c_{W W W} / \Lambda^{2}, c_{B} / \Lambda^{2}$ and $c_{W} / \Lambda^{2}$ derived from the leading lepton $p_{\mathrm{T}}$ spectrum in $W^{+} W^{-}$production at $8 \mathrm{TeV}$ from ATLAS [103] and CMS [104]

parameters with the assumption of no form factor. The observedEFT parameters are compared for both experiments in Table 8. The ATLAS and CMS limits show a similar sensitivity to EFT parameters for a comparable data set.

\subsubsection{The $W^{ \pm} Z$ process, leptonic channel}

The $W^{ \pm} Z$ di-bosons process has a production cross section of roughly $1 / 3$ relative to the $W^{+} W^{-}$ process. $W^{ \pm} Z$ pairs are produced from quark-antiquark initial states at leading order and quark-gluon initial states at next-to-leading order. The SM-allowed $s$-channel is sensitive to anomalous TGC. In the leptonic channel the clean signature of three isolated leptons allows to effectively suppress background processes with leading background contributions from $Z Z, Z+$ jets, and from top quark production.

The ATLAS and CMS experiments measured the $W^{ \pm} Z$ production cross section in the fully leptonic decay channel $\ell^{+} \ell^{-} \ell \nu$ at $\sqrt{s}=7 \mathrm{TeV}[105,106], \sqrt{s}=8 \mathrm{TeV}[107,106]$ and $\sqrt{s}=13 \mathrm{TeV}[108$, 109] and set limits on charged anomalous TGC and EFT parameters. Generally, unfolded differential distributions in different kinematic variable are also provided.

The $W^{ \pm} Z$ channel allows to measure the helicity fractions of the $Z$ and $W^{ \pm}$polarisation, and with the full run-2 completed a sufficiently large dataset is available to measure the helicity fraction to a few percent precision. Due to the massiveness the $W^{ \pm}$and $Z$ boson have besides the transverse left and right 
handed polarisation also a longitudinal polarisation state. Thus the polarisation fractions provide an additional precision measurement to test the SM prediction of the subtle gauge cancellation behaviour in the non-Abelian gauge sector. EFT or anomalous TGC would alter the polarisation fractions. The $W^{ \pm} Z$ channel was the first at LHC to exploit the helicity fraction in di-boson pairs [108]. Previously, experiments at LEP had already studied the spin structure in $W^{+} W^{-}$pair production [1] to derive limits on anomalous CP violating triple gauge couplings. A prospect that should also be valid for the LHC experiments, but so far CP violating couplings have not been investigated in the di-boson process at LHC.

In the fully leptonic analyses the boson decays to electrons or muons are considered as signal, whereas the tauonic decays are treated as background. The dominant background sources are $Z+$ jet where the decay products of the jet are misidentified as a lepton that passes all isolation and quality requirement cuts, and $Z Z$ production. Both processes together account for about $40 \%$ to $70 \%$ of the overall background. The overall signal to background ratio is about 4 . The leading systematic uncertainty is related to the data-driven estimation method of the $Z+$ jet background, followed by uncertainties on the $E_{\mathrm{T}}^{\text {miss }}$ determination. The total relative systematic uncertainty is around $4 \%$ to $7 \%$.

The fiducial cross section for the $7 \mathrm{TeV}\left(8\right.$ and $13 \mathrm{TeV}$ ) ATLAS analysis is defined by $p_{\mathrm{T}}^{\mu, e}>15 \mathrm{GeV}$ for the leptons from the $Z$ decay, $p_{\mathrm{T}}^{\mu, e}>20 \mathrm{GeV}$ for the lepton from the $W,\left|\eta^{\mu, e}\right|<2.5, p_{\mathrm{T}}^{\nu}>25 \mathrm{GeV}$, $\left|m_{l l}-m_{Z}\right|<10(25) \mathrm{GeV}, M_{T}^{W}>20(30) \mathrm{GeV}$ and $\Delta R>0.3$ for the three possible $\ell \ell$ pairings. The total cross section requires the mass of the $Z$ to be in the range of $66 \mathrm{GeV}<\left|m_{Z}\right|<116 \mathrm{GeV}$ to suppress the contribution from $\gamma^{*}$. CMS uses a different definition for their $13 \mathrm{TeV}$ measurement of a fiducial cross section requiring three leptons within $\left|\eta^{l}\right|<2.5(2.4)$ for electrons (muons), including electrons or muons from tau decays from the signal process. Further $p_{\mathrm{T}}^{l_{Z 1}}>25 \mathrm{GeV}, p_{\mathrm{T}}^{l_{Z 2}}>10 \mathrm{GeV}$, and $p_{\mathrm{T}}^{l_{W 1}}>25 \mathrm{GeV}$ are required. The tri-lepton invariant mass must be above $100 \mathrm{GeV}$ and the $Z$ mass within $15 \mathrm{GeV}$ of the pole mass. The different definitions of fiducial cross section between experiments and at different $\sqrt{s}$ make a direct comparison problematic, but facilitate nevertheless comparisons to theoretical calculations. The total cross sections are compared to the SM expectation at NLO in Table 9. Overall good agreement between experimental cross section and the latest calculation at (N)NLO are observed. The total cross sections as a function of $\sqrt{s}$ and the normalized unfolded $p_{\mathrm{T}}^{Z}$ spectrum is compared to the NLO calculation of MCONLO [65] in Figure 9, showing good agreement with the SM prediction.

\begin{tabular}{|c|c|c|c|c|c|}
\hline Experiment & cross section & $\sqrt{s}[\mathrm{TeV}]$ & measured & predicted & reference \\
\hline ATLAS & fiducial & 7 & $92_{-6}^{+7}$ (stat.) \pm 4 (syst.) \pm 2 (lumi.) fb & - & [105] \\
\hline CMS & total & 8 & $24.1 \pm 0.9$ (stat.) \pm 1.4 (syst.) \pm 0.8 (theo.) \pm 0.6 (lumi.) pb & $23.7 \pm 0.4 \mathrm{pb}(\mathrm{NNLO})$ & {$[106]$} \\
\hline ATLAS & fiducial & 8 & $35 \pm 0.9$ (stat.) \pm 0.8 (syst.) \pm 0.8 (lumi.) $\mathrm{fb}$ & $30 \pm 0.5(\mathrm{PDF}) \pm 0.8$ (scale) fb & {$[107]$} \\
\hline ATLAS & total & 13 & $51 \pm 0.8$ (stat.) \pm 1.8 (syst.) \pm 1.1 (lumi.) pb & $49_{-10}^{+1.1} \mathrm{pb}(\mathrm{NNLO})$ & {$[108]$} \\
\hline CMS & total & 13 & $48.1 \pm 1.0$ (stat.) ${ }_{-2.2}^{+2.4}$ (syst.) \pm 0.4 (theo.) \pm 1.4 (lumi.) $\mathrm{pb}$ & $50.0 \pm 1.0 \mathrm{pb}(\mathrm{NNLO})$ & {$[109]$} \\
\hline
\end{tabular}

Table 9: Summary of measured total and fiducial $W^{ \pm} Z$ production cross sections from ATLAS and CMS at 7, 8 , and $13 \mathrm{TeV}$ centre-of-mass energies in the $\ell^{+} \ell^{-} \ell \nu$ final state, and comparison to (N)NLO calculations. Note that the definition of fiducial cross section differ between experiments and $\sqrt{s}$ energies and are not directly comparable.

In the most recent analysis limits on the EFT parameters $c_{W} / \Lambda^{2}, c_{W W W} / \Lambda^{2}$ and $c_{B} / \Lambda^{2}$ are extracted from the tri-lepton invariant mass distribution. Previously, also the transverse momentum of the $Z$, $p_{\mathrm{T}}^{Z}[105]$, or the transverse mass of the $W Z$ system [107] were used, which all show similar sensitivity 


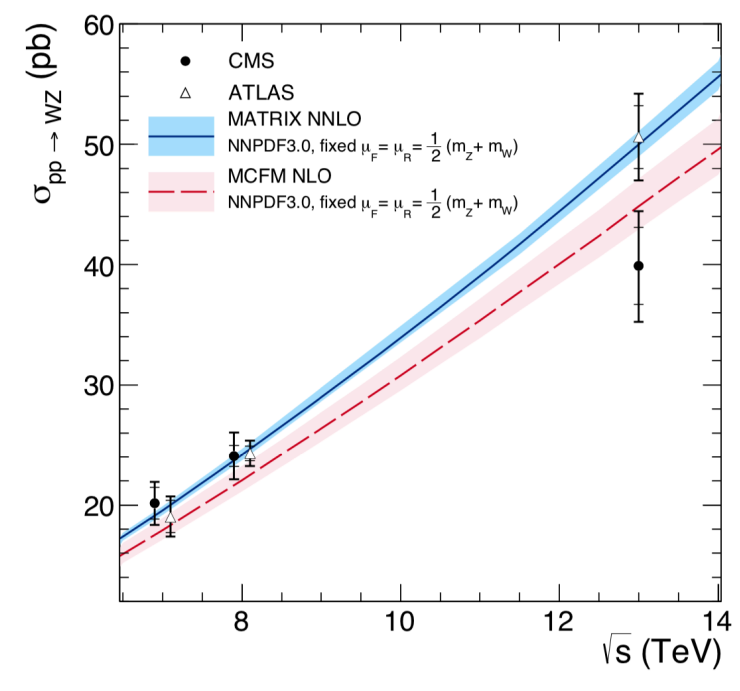

(a)

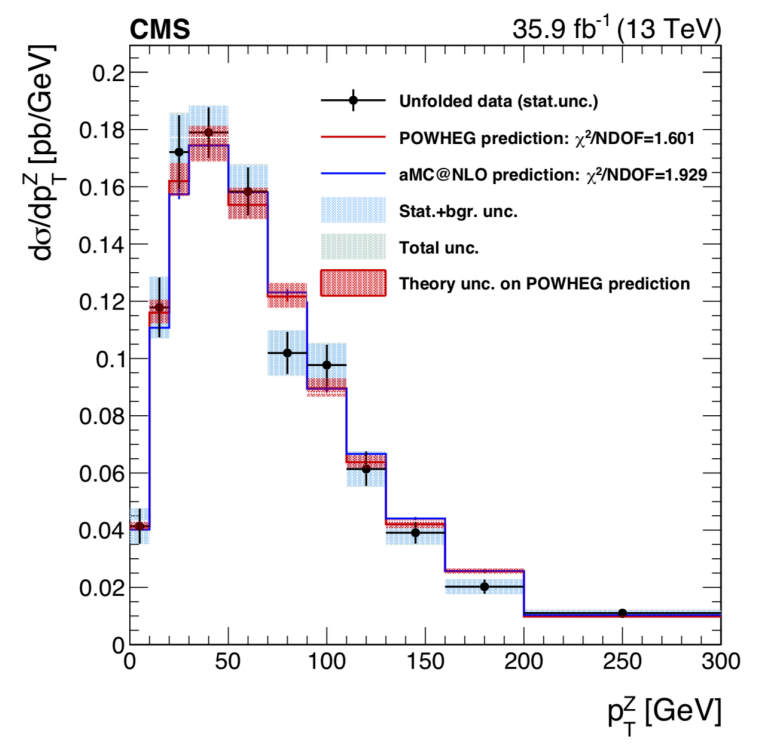

(b)

Figure 9: (a) The $W^{ \pm} Z$ total cross section for different $\sqrt{s}$ from CMS and ATLAS compared to NLO and NNLO calculations. The data points include statistical (inner bar) and full uncertainty (outer bar). The theoretical calculation include the uncertainty band due to hadronization and factorisation scale variations and PDF variations (from [106]). (b) The differential distribution in $p_{\mathrm{T}}$ of the $Z$ boson for $\sqrt{s}=13 \mathrm{TeV}$ measured by CMS [109] and unfolded for detector effects. Overlaid is a comparison to an NLO calculation.

\begin{tabular}{ccc}
\hline Coupling & Expected $\left[\mathrm{TeV}^{-2}\right]$ & Observed $\left[\mathrm{TeV}^{-2}\right]$ \\
\hline$c_{W} / \Lambda^{2}$ & {$[-3.3,2.0]$} & {$[-4.1,1.1]$} \\
$c_{W W W} / \Lambda^{2}$ & {$[-1.8,1.9]$} & {$[-2.0,2.1]$} \\
$c_{B} / \Lambda^{2}$ & {$[-130,170]$} & {$[-100,160]$} \\
\hline
\end{tabular}

Table 10: Expected and observed 95\% CL limits on $c_{W} / \Lambda^{2}, c_{W W W} / \Lambda^{2}$ and $c_{B} / \Lambda^{2}$ obtained with 13 $\mathrm{TeV}$ dataset from CMS [109].

to anomalous TGC.

The EFT parameter $95 \%$ CL limits on $c_{W} / \Lambda^{2}, c_{W W W} / \Lambda^{2}$ and $c_{B} / \Lambda^{2}$ obtained with $13 \mathrm{TeV}$ dataset from CMS [109] are shown in Table 10. Similar constraints in the anomalous TGC formalism are available from ATLAS and show a comparable sensitivity [108].

The polarisation of vector bosons can be expressed with the parameters $f_{L}, f_{R}$, and $f_{0}$, representing the diagonal elements of the spin-density matrix [110], i.e. the polarisation fractions of left and right handed transverse polarisation $\left(f_{L}, f_{R}\right)$ and the longitudinal component $\left(f_{0}\right)$. The polarisation state influences the azimuthal decay angle of emitted leptons with respect to the direction of flight of the vector boson, in the rest frame of boson. The definition of decay angles is schematically shown in Figure 10(a). The distribution of $\cos \theta_{l}$ from the $W^{+}$in $W^{ \pm} Z$ events as shown in Figure 10 is of the form $f_{L} \frac{3}{8}(1+\cos \theta)^{2}+f_{L} \frac{3}{8}(1-\cos \theta)^{2}+f_{0} \frac{3}{4}(\sin \theta)^{2}$ at Born level for the total phase space. For the fiducial phase space the polarisation fractions are determined from template fits with three samples corresponding to the three pure polarisation states. Since $f_{L}+f_{R}+f_{0}=1$ two degrees of freedom are left, and the polarisation fractions are measured in terms of $f_{0}$ and $f_{L}-f_{R}$, as seen in Table 11 where the $13 \mathrm{TeV}$ results of ATLAS are compared to NNLO calculations. Within uncertainties the polarisation fraction are consistent with the SM. 


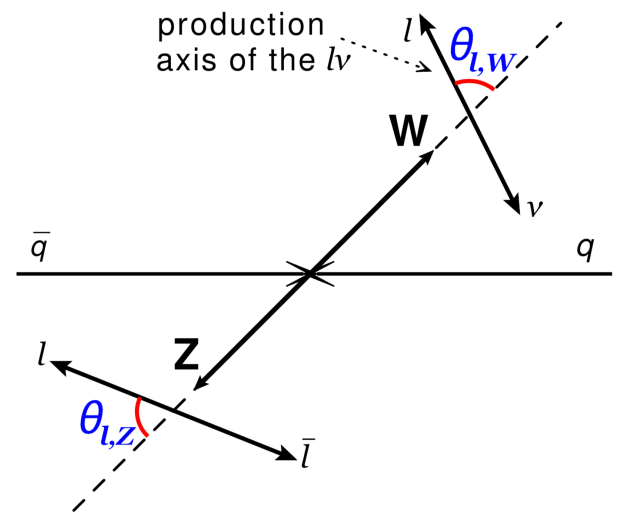

(a)

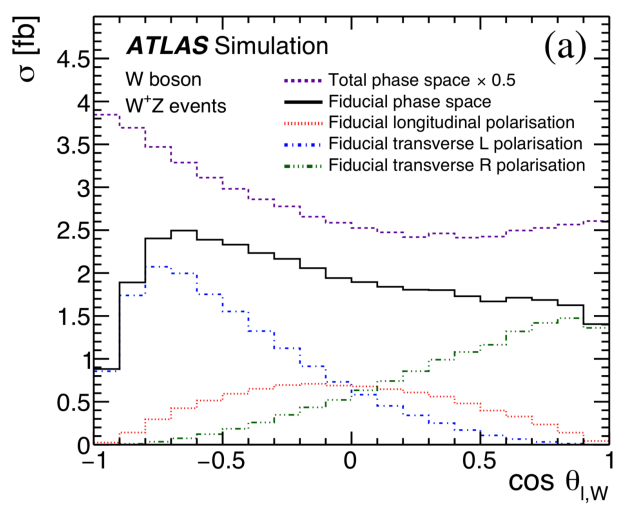

(b)

Figure 10: (a) Sketch of the decay angle definition in leptonic $W^{ \pm} Z$ decays [108]. The angles are measured between the direction of flight of the boson in the di-boson rest frame and the lepton direction of flight in the boson rest frame. (b) Distribution of $\cos \theta_{l}$ from the $W^{+}$in $W^{ \pm} Z$ events for the total and fiducial phase space, and template distributions for pure transverse left and right handed, and longitudinal polarisation states in the fiducial volume [108].

\begin{tabular}{ccccc}
\hline Channel & $f_{0}$ (observed) & $f_{0}$ (predicted) & $f_{L}-f_{R}$ (observed) & $f_{L}-f_{R}$ (predicted) \\
\hline$W^{+}$ & $0.26 \pm 0.08$ & $0.2448 \pm 0.0010$ & $-0.02 \pm 0.04$ & $0.0868 \pm 0.0014$ \\
$Z\left(W^{+}\right)$ & $0.27 \pm 0.05$ & $0.2401 \pm 0.0014$ & $-0.32 \pm 0.21$ & $-0.262 \pm 0.009$ \\
$W^{-}$ & $0.32 \pm 0.09$ & $0.2651 \pm 0.0015$ & $-0.05 \pm 0.05$ & $-0.034 \pm 0.004$ \\
$Z\left(W^{-}\right)$ & $0.21 \pm 0.06$ & $0.2389 \pm 0.0015$ & $-0.46 \pm 0.25$ & $0.0468 \pm 0.0034$ \\
\hline
\end{tabular}

Table 11: Observed and predicted helicity fraction $f_{0}$ and $f_{L}-f_{R}$ as measured in $W^{ \pm} Z$ events by ATLAS [108]. The predictions are calculated at in NNLO [52]. A fiducial phase space definition is used to select the events. 


\subsubsection{The $Z Z$ process, leptonic channel}

The $Z Z$ process has been one of the first to be measured at the LHC, despite its relatively low production cross section, about one order of magnitude lower compared to the $W^{+} W^{-}$process. However, all leptonic decay products are accessible and invariant mass constraints on the $Z$ mass make this process almost background free. The SM process is an important and irreducible background to searches for di-boson resonances and Higgs production in the $Z Z$ decay channel. At leading order the production cross section is dominated by quark anti-quark annihilation in the $t$ and $u$-channel, while the $s$-channel process is forbidden in the SM as it would require a neutral triple gauge boson vertex, which violates the underlying $S U_{L}(2) \otimes U_{Y}(1)$ gauge symmetry. The contribution from triangular fermion loops at the one loop level are on the order of $10^{-4}$ in terms of effective TGC [111]. The gluon fusion process contributes about $\mathrm{O}(10 \%)$ [112] to the total production cross section. The Higgs contribution to $Z Z$ is negligible in the case where both $Z$ bosons are required to be on-shell.

The four lepton final state provides an almost background free signature but at the expense of a relatively small branching ratio of $\mathcal{B}(Z Z) \rightarrow \ell^{+} \ell^{-} \ell^{+} \ell^{-}=0.101^{2} \cdot \frac{4}{9}=0.0045$ [113] when using only the electronic and muonic decay channels of the $Z$. The branching ratio is one order-of-magnitude larger when taking the decay into a pair of neutrinos of one $Z$ into account giving a branching ratio of $\mathcal{B}\left(Z Z \rightarrow \ell^{+} \ell^{-} \nu \bar{\nu}\right)=0.101 \cdot 0.20 \cdot 2 \cdot \frac{2}{3}=0.0269$, in the di-lepton and missing energy channel. The disadvantage in this channel is the relatively large background.

The ATLAS collaboration has published results on the full $7 \mathrm{TeV}$ and $8 \mathrm{TeV}$ data-set in the $\ell^{+} \ell^{-} \ell^{+} \ell^{-}$ and $\ell^{+} \ell^{-} \nu \bar{\nu}$ final state $[114,115]$, and at $13 \mathrm{TeV}$ in the $\ell^{+} \ell^{-} \ell^{+} \ell^{-}$final state [116]. Similarly, the CMS collaboration has analysed the full $7 \mathrm{TeV}$ and $8 \mathrm{TeV}$ data sets in both the $\ell^{+} \ell^{-} \ell^{+} \ell^{-}[117,118]$ and $\ell^{+} \ell^{-} \nu \bar{\nu}$ final state [119], and the $\ell^{+} \ell^{-} \ell^{+} \ell^{-}$final state at $13 \mathrm{TeV}[120]$.

Theoretical predictions for $Z Z$ production are available at NLO [121] (including the $g q$ contribution) and NNLO in $\alpha_{s}[112,50]$ (including the $g g$ contribution). The $g g$ contribution is of the order $\mathrm{O}(10 \%)$. Electroweak corrections at NLO have also been calculated [122, 123, 51].

The event selection for the $\ell^{+} \ell^{-} \ell^{+} \ell^{-}$final state requires exactly four leptons, $l=e, \mu$, fulfilling a set of cuts on kinematic quantities. CMS included in the analysis of the 7 and $8 \mathrm{TeV}$ data-set the $Z \rightarrow \tau^{+} \tau^{-}$channel with subsequent hadronic and leptonic $\tau$ decays. ATLAS uses in addition forward leptons outside the ID tracker to increase the acceptance by $6 \%$ for electrons and $10 \%$ for muons. The $\ell^{+} \ell^{-} \ell^{+} \ell^{-}$channels offers the cleanest event sample with a background level of only $2-3 \%$ from $Z+$ jets, $t \bar{t}$, and di-boson events. The background is estimated from data in control regions with looser selection criteria.

Events in the $\ell^{+} \ell^{-} \nu \bar{\nu}$ final state are characterized by exactly two leptons and missing energy. The event selection requires a $Z \rightarrow \ell^{+} \ell^{-}$candidate and missing energy in the event. Both experiments use refined observables of missing energy with additional information to improve the rejection against instrumental background. The background level is of the same order as the signal and substantially higher than for the $\ell^{+} \ell^{-} \ell^{+} \ell^{-}$channel. Main background sources are $V+j e t s$, and di-boson production. ATLAS and CMS use data driven techniques to constrain the dominant background sources.

Besides the total cross section for the $p p \rightarrow Z Z$ production process, both experiments measure also fiducial and differential cross sections. The results are summarized in Table 12 and Figure 11. ATLAS and CMS use different definitions of the fiducial phase thus a direct comparison is not possible. For the total cross section definition the two experiments differ slightly in the $Z$ mass range, where CMS prefers a wider range of $60 \mathrm{GeV}<m_{Z}<120 \mathrm{GeV}$ than ATLAS with $66 \mathrm{GeV}<m_{Z}<116 \mathrm{GeV}$. This difference contributes to the variation in the quoted predicted cross section. Good agreement between experimental and theoretical cross section values is observed in both ATLAS and CMS analyses.

Anomalous TGC and EFT parameters typically enhance the cross section at high partonic centre of

mass, $\sqrt{\hat{s}}$. Consequently, differential distributions with strong dependence on $\sqrt{\hat{s}}$ are well suited to set limits on these parameter, e.g. for the $\ell^{+} \ell^{-} \ell^{+} \ell^{-}$and $\ell^{+} \ell^{-} \nu \bar{\nu}$ final state of $Z Z$ production the invariant 


\begin{tabular}{|c|c|c|c|c|c|} 
Experiment & decay channel & $\sqrt{s}[\mathrm{TeV}]$ & measured $\sigma_{\text {total }}[\mathrm{pb}]$ & predicted $\sigma_{\text {total }}[\mathrm{pb}]$ & reference \\
\hline ATLAS & $\ell^{+} \ell^{-} \ell^{+} \ell^{-}, \ell^{+} \ell^{-} \nu \bar{\nu}$ & 7 & $6.7 \pm 0.7$ (stat.) ${ }_{-0.3}^{+0.4}$ (syst.) \pm 0.3 (lumi.) & $6.18_{-0.18}^{+0.25}$ \\
CMS & $\ell^{+} \ell^{-} \ell^{+} \ell^{-}$ & 7 & $6.2_{-0.8}^{+0.9}$ (stat.) ${ }_{-0.3}^{+0.4}$ (syst.) \pm 0.1 (lumi.) & $6.3 \pm 0.4$ \\
CMS & $\ell^{+} \ell^{-} \nu \bar{\nu}$ & 7 & $5.1_{-1.4}^{+1.5}$ (stat.) ${ }_{-1.1}^{+1.4}$ (syst.) \pm 0.1 (lumi.) & {$[117]$} \\
ATLAS & $\ell^{+} \ell^{-} \ell^{+} \ell^{-}, \ell^{+} \ell^{-} \nu \bar{\nu}$ & 8 & $7.3 \pm 0.4$ (stat.) \pm 0.3 (syst.) ${ }_{-0.1}^{+0.2}$ (lumi.) & $6.2_{-0.2}^{+0.3}$ \\
CMS & $\ell^{+} \ell^{-} \ell^{+} \ell^{-}$ & 8 & $7.7 \pm 0.5$ (stat.) $)_{-0.4}^{+0.5}$ (syst.) \pm 0.2 (lumi.) & $6.6_{-0.6}^{+0.7}$ \\
CMS & $\ell^{+} \ell^{-} \nu \bar{\nu}$ & 8 & $7.2 \pm 0.8$ (stat.) ${ }_{-1.5}^{+1.9}$ (syst.) \pm 0.2 (lumi.) & $7.7 \pm 0.6$ \\
ATLAS & $\ell^{+} \ell^{-} \ell^{+} \ell^{-}$ & 13 & $17.3 \pm 0.6$ (stat.) \pm 0.5 (syst.) \pm 0.6 (lumi.) & $7.6_{-0.3}^{+0.4}$ \\
CMS & $\ell^{+} \ell^{-} \ell^{+} \ell^{-}$ & 13 & $17.2 \pm 0.5$ (stat.) \pm 0.7 (syst.) \pm 0.4 (theo.) \pm 0.4 (lumi.) & $16.9_{-0.5}^{+0.6}$ & $16.9_{-0.5}^{+0.6}$
\end{tabular}

Table 12: Summary of measured $Z Z$ production cross sections from ATLAS and CMS at 7, 8 and 13 $\mathrm{TeV}$ centre-of-mass energies in the four lepton and $\ell^{+} \ell^{-} \nu \bar{\nu}$ final state.

\begin{tabular}{|c|c|c|}
\hline Parameter & $\begin{array}{c}\text { ATLAS } \\
\text { Observed }\end{array}$ & $\begin{array}{c}\text { CMS } \\
\text { Observed }\end{array}$ \\
\hline$f_{40}^{\gamma} 10^{-3}$ & {$[-2.4,2.4]$} & {$[-1.2,1.3]$} \\
$f_{40}^{Z} 10^{-3}$ & {$[-1.5,1.5]$} & {$[-1.2,1.0]$} \\
$f_{50}^{\gamma} 10^{-3}$ & {$[-1.8,1.8]$} & {$[-1.2,1.3]$} \\
$f_{50}^{Z} 10^{-3}$ & {$[-1.5,1.5]$} & {$[-1.2,1.3]$} \\
$c_{\tilde{B} W} / \Lambda^{2}\left[\mathrm{TeV}^{-4}\right]$ & {$[-5.9,5.9]$} & - \\
$c_{W W} / \Lambda^{2}\left[\mathrm{TeV}^{-4}\right]$ & {$[-3.0,3.0]$} & - \\
$c_{B W} / \Lambda^{2}\left[\mathrm{TeV}^{-4}\right]$ & {$[-3.3,3.3]$} & - \\
$c_{B} / \Lambda^{2}\left[\mathrm{TeV}^{-4}\right]$ & {$[-2.7,2.8]$} & - \\
\hline
\end{tabular}

Table 13: $95 \%$ CL limits on the anomalous TGC $f_{40}^{\gamma}, f_{40}^{Z}, f_{50}^{\gamma}, f_{50}^{\gamma}$, and EFT parameters $c_{B W} / \Lambda^{2}$, $c_{W W} / \Lambda^{2}$ and $c_{B} / \Lambda^{2}$ obtained with $13 \mathrm{TeV}$ dataset from the ATLAS [116] and CMS [109] experiments in the $\ell^{+} \ell^{-} \ell^{+} \ell^{-}$final state.

di-boson mass (four lepton channel), the transverse momentum of the leading lepton $\left(\ell^{+} \ell^{-} \nu \bar{\nu}\right.$-channel), or the transverse momentum of the leading $Z$ (all channels). Limits are determined in the framework of the vertex function parametrization with coefficients of the form $f_{i}^{V}$ as defined in reference [124], and for the more recent results at $\sqrt{s}=13 \mathrm{TeV}$ also in the EFT approach [32]. A comparison of the measured and predicted differential cross sections in bins of the leading $Z$ transverse momentum from ATLAS, and the four-lepton invariant mass from CMS is shown in Figure 14. The prediction for the case of a non-zero value of anomalous coupling parameters is shown in the invariant mass spectrum. The typical enhancement over the SM value, increasing at high invariant masses is evident. The overall good agreement with SM calculations is leading to stringent limits on anomalous TGC parameters.

Both experiments publish 95\% CL limits on anomalous TGC without form factors in the $\ell^{+} \ell^{-} \ell^{+} \ell^{-}$ and $\ell^{+} \ell^{-} \nu \bar{\nu}$ channels and EFT parameters. No inconsistencies with the SM are observed and the most recent and stringent limits obtained with the $13 \mathrm{TeV}$ data-set are summarised in Table 13. The limits obtained with the combination of $\sqrt{s}=7 \mathrm{TeV}$ and $\sqrt{s}=8 \mathrm{TeV}$ data-sets of about $25 \mathrm{fb}^{-1}$, combining $\ell^{+} \ell^{-} \ell^{+} \ell^{-}$and $\ell^{+} \ell^{-} \nu \bar{\nu}$ final states [119] from CMS are about factor two less stringent than the current limits obtained with the $36 \mathrm{fb}^{-1}$ data-set at $13 \mathrm{TeV}$ and using only the $\ell^{+} \ell^{-} \ell^{+} \ell^{-}$channel. This is expected as a consequence of the strong dependence of the cross section enhancement due to anomalous TGC with the partonic centre of mass energy $\hat{s}$.

The limit results obtained at LHC are driven by the steep increase of sensitivity with higher centreof-mass energy and are about 2 orders-of-magnitude more stringent compared to the combined LEP result [93]. 


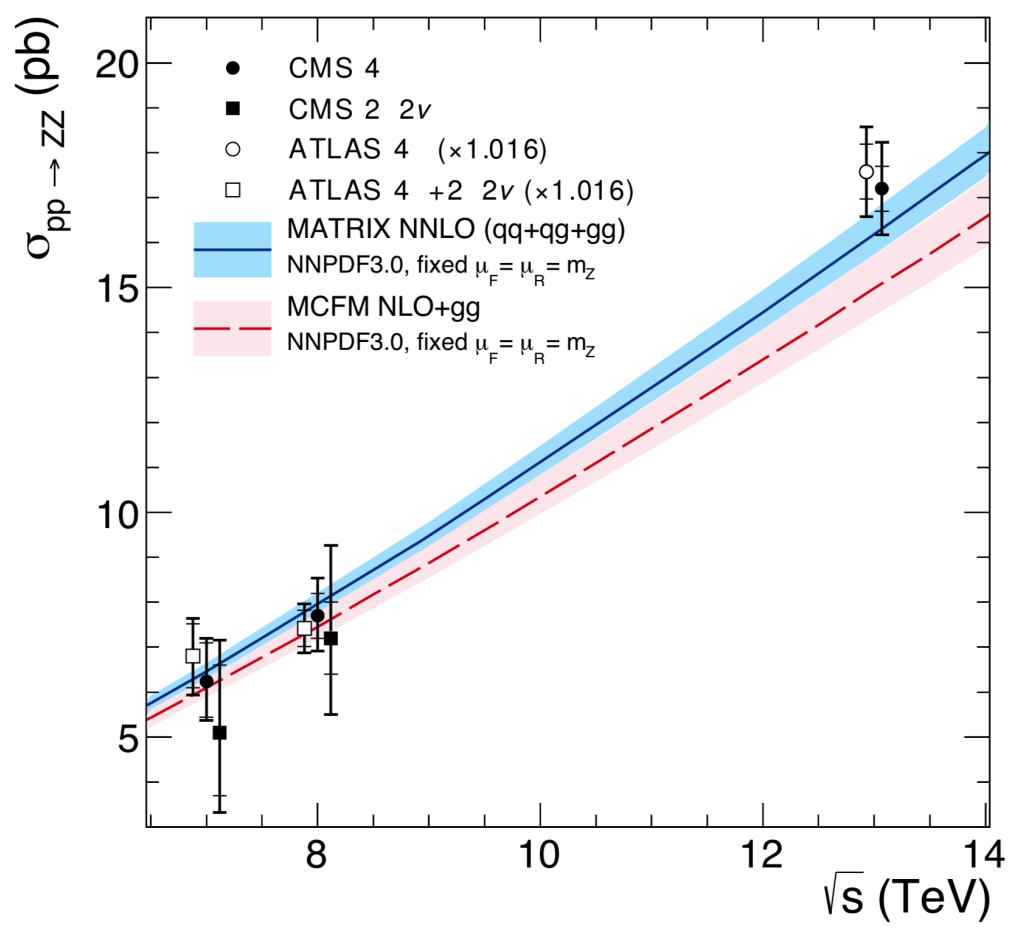

Figure 11: The total $Z Z$ cross section as a function of $\sqrt{s}$ as measured by the ATLAS and CMS experiments and compared to calculation at (N)NLO from [120]. In this plot the ATLAS measurements are corrected by $1.6 \%$ to account for the difference in the $Z$ mass window definition between ATLAS (66-116 GeV) and CMS (60-120GeV).

\subsubsection{The $W^{ \pm} V$ process, semi leptonic channel}

The semileptonic decay channel of $W^{ \pm} V$ process with $V=W^{ \pm}, Z$ decaying into a pair of quarks, and the $W^{ \pm}$leptonically, has the advantage of a higher branching ratio to quarks compared to leptons $(e, \mu)$ of approximately factor 10 for the $Z$ and factor 3 for the $W^{ \pm}$. In the case of $W^{+} W^{-}$production the hadronically decaying $W^{ \pm}$also allows to reconstruct the transverse momenta of the two $W^{ \pm}$bosons, which is directly accessible in the fully leptonic decay channel. The advantages are to some degree offset by the increased background level from QCD background. However, the increased statistics of signal events at high partonic centre-of-mass $\hat{s}$ compared to the fully leptonic decay modes, and the decreasing background levels in this kinematic region, enhances specifically the sensitivity to anomalous TGC. The $W^{ \pm} V \rightarrow \ell^{ \pm} \nu q \bar{q}$ final state is identified by a single isolated lepton, two jets, and missing transverse energy in the detector. In the kinematic region of high $\hat{s}$ the bosons are strongly boosted, leading to a narrowing opening angle of the two jets. To improve the acceptance the analysis distinguish between two separately reconstructed jets $(\ell \nu j j)$ and merged jet $(\ell \nu J)$ categories.

ATLAS and CMS have published measurements of the inclusive production cross section $W^{+} W^{-}+$ $W^{ \pm} Z$ and set limits on charged anomalous TGC with the full data set of the $\sqrt{s}=7 \mathrm{TeV}[125,126]$ and $\sqrt{s}=8 \mathrm{TeV}$ run $[127,128]$. The semileptonic decay channel covers electronic or muonic decays of the $W^{ \pm}$. The event selection requirements vary between different data-sets and between experiments. The cut ranges for the lepton $p_{\mathrm{T}}$ and $E_{\mathrm{T}}^{\text {miss }}$ range from $40 \mathrm{GeV}$ to $70 \mathrm{GeV}$. Additional requirements are on the jet multiplicity, i.e. exactly two jets or exactly on large radius jet and no additional jets. The mass of the hadronic system is used to fit template distributions of background and signal to extract the yield of signal events. Additional topological selection criteria on the separation of jet(s) and lepton 


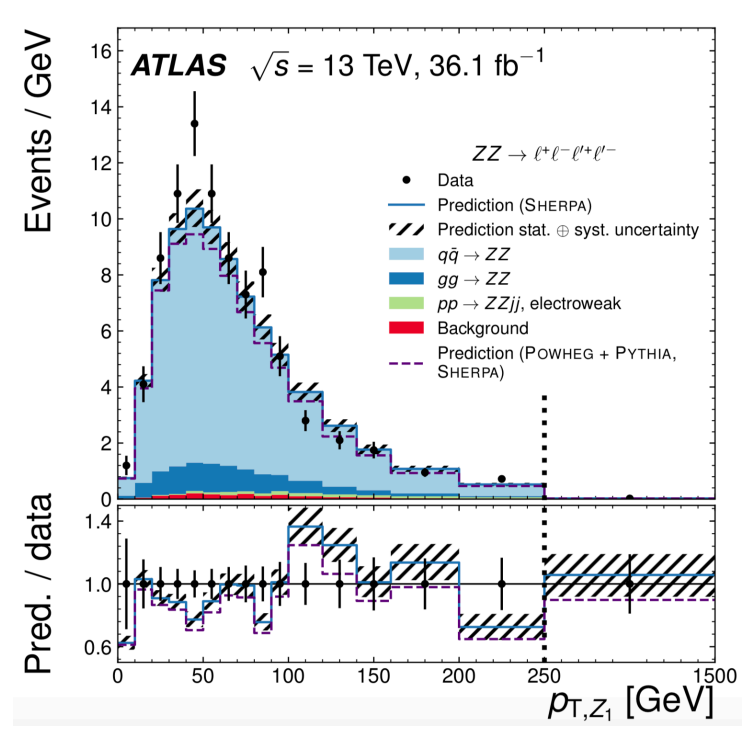

(a)

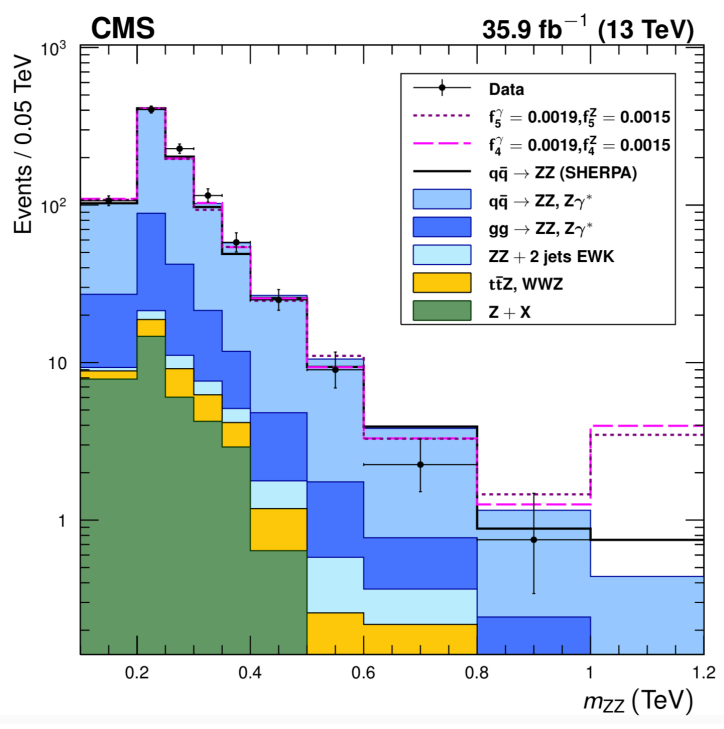

(b)

Figure 12: a) Transverse momentum distribution of the leading $Z$ boson measured with the ATLAS detector at $\sqrt{s}=13 \mathrm{TeV}$ compared to to predictions of different calculations [116], with uncertainties including statistical and systematic contributions added in quadrature. For better visibility the $x$-axis scale is changed at the last bin, indicated with the dotted line. (b) Distribution of the four-lepton invariant mass measured at $\sqrt{s}=13 \mathrm{TeV}$ with the CMS detectors compared to SM prediction (solid line) and with samples including anomalous TGC signal examples (dashed line) [120]. The last bin includes events with masses above $1.2 \mathrm{TeV}$.

aim to further reduce the background of top pair production and $V$ with associated jets production. The signal fraction of $W^{+} W^{-}+W^{ \pm} Z$ events after selection is about $2 \%$ in the $\ell \nu j j$ channel, and about $5-8 \%$ in the $\ell \nu J$ channel and no further selection criteria are applied to distinguish between $W^{+} W^{-}$ and $W^{ \pm} Z$ production. The backgrounds from dominant $Z / W^{ \pm}+$jets production, and the sub-leading background contributions from $t \bar{t}$ and multi-jet production is constrained with data driven methods by fitting background templates to control regions with orthogonal cuts to the signal regions and enriched for the respective background contribution. The control region samples are almost signal free and allow to obtain the background normalisation as shown in Figure 13 from the ATLAS 8 TeV analysis [127] for the $W^{ \pm}+$jets and top quark control regions .

The signal fraction is fitted in the di-jet invariant mass $(\ell \nu j j)$ and jet mass $(\ell \nu J)$ distribution (see Figure 13), with the normalisation of leading background contributions constrained by the control regions. The largest systematic uncertainty is related to the modelling of the $Z / W^{ \pm}+$jets background with about $20-60 \%$ uncertainty for the cross section. The measured and predicted cross sections are compared in Table 14.

\begin{tabular}{|c|c|c|c|c|c|} 
Experiment & cross section & $\sqrt{s}[\mathrm{TeV}]$ & measured $[\mathrm{pb}]$ & predicted $[\mathrm{pb}]$ & reference \\
\hline ATLAS & total & 7 & $68 \pm 7$ (stat.) \pm 19 (syst.+lumi.) & $61.1 \pm 2.2$ & {$[125]$} \\
CMS & total & 7 & $68.9 \pm 8.7$ (stat.) \pm 9.7 (syst.) \pm 1.5 (lumi.) & $65.6 \pm 2.2$ & {$[126]$} \\
ATLAS & fiducial & 8 & $209 \pm 28$ (stat.) \pm 45 (syst.) & $225 \pm 13$ & {$[127]$}
\end{tabular}

Table 14: Summary of measured total $W^{ \pm} Z+W^{+} W^{-}$production cross sections from ATLAS and CMS in the $W^{ \pm} V \rightarrow \ell^{ \pm} \nu q \bar{q} \quad$ final state.

One of the main motivations to analyse the semi-leptonic decays of di-bosons is the enhanced sensi- 


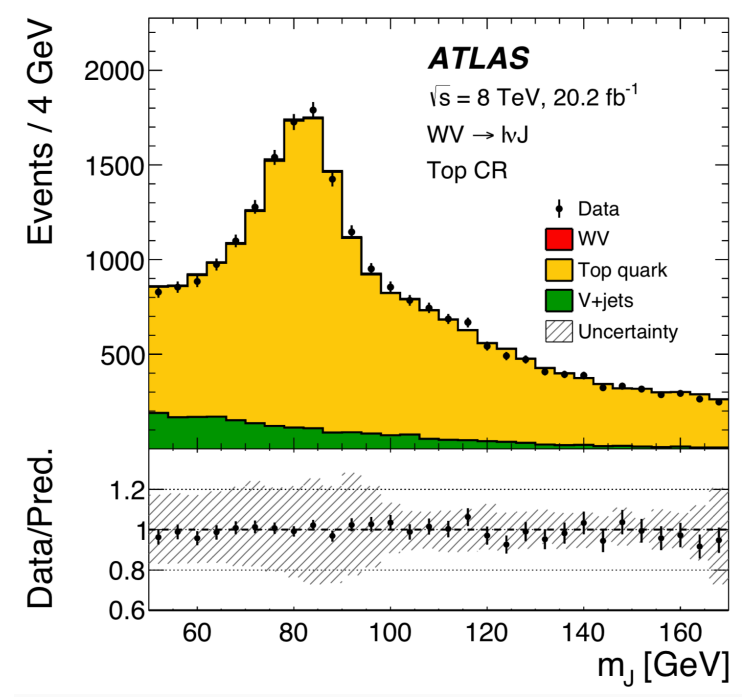

(a)

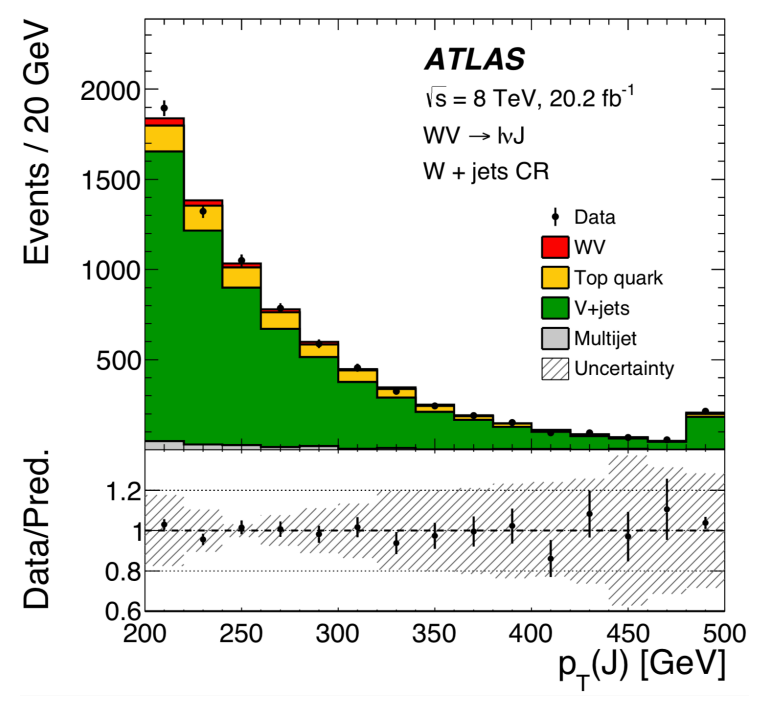

(b)

Figure 13: Comparison of data and prediction for the $\ell \nu J$ channel in a) the Jet mass distribution in the top quark control region and (b) $p_{\mathrm{T}}(J)$ distribution in the $W^{ \pm}+$jets control region after applying a scale factor. The plots are from the ATLAS measurement at $8 \mathrm{TeV}$ [127].

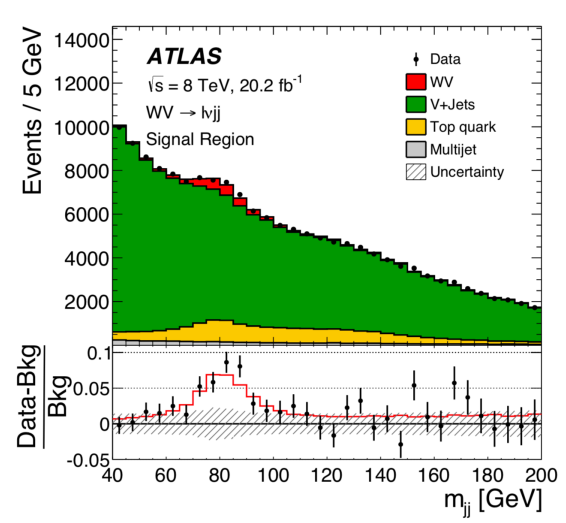

(a)
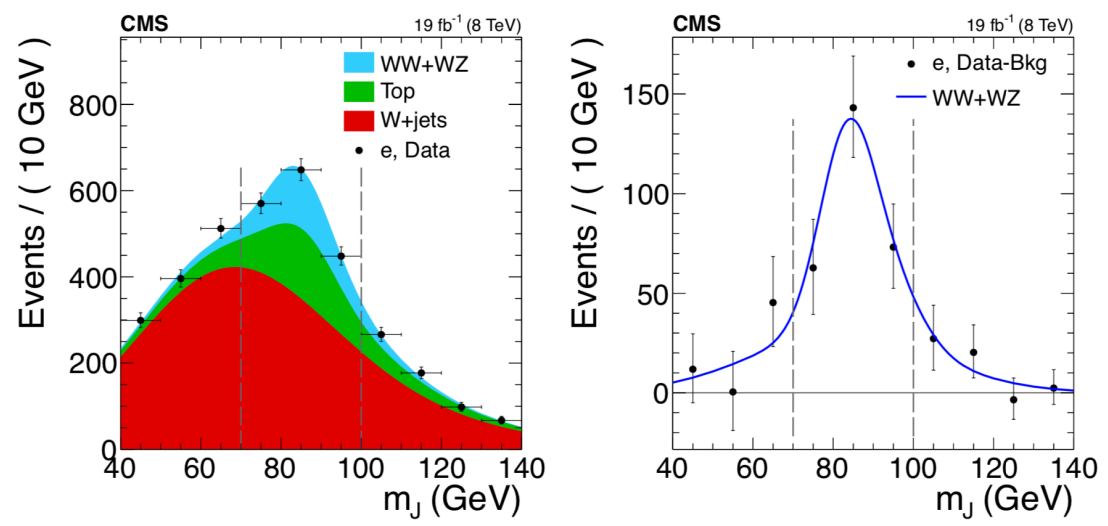

(b)

Figure 14: (a) The invariant mass distribution of the di-jet system in the $\ell \nu j j$ channel in the signal region observed in data from the $8 \mathrm{TeV}$ ATLAS measurement [127]. Also shown are the background and signal estimates after fitting the background normalisation. (b) Distribution of the jet-mass in the $e \nu J$ channel after fitting the background and signal fractions to the data from the 8 TeV CMS analysis [128]. The distribution is shown for all events (left) and after subtraction of the fitted background prediction (right). 


\begin{tabular}{cccccc}
\hline & & \multicolumn{2}{c}{ CMS } & \multicolumn{2}{c}{ ATLAS } \\
Parameter & $\sqrt{s}[\mathrm{GeV}]$ & Observed & Expected & Observed & Expected \\
\hline$\lambda_{Z}=\lambda_{\gamma}$ & 7 & - & {$[-0.038,0.030]$} & {$[-0.039,0.040]$} & {$[-0.048,0.047]$} \\
$\Delta \kappa_{\gamma}$ & 7 & - & {$[-0.11,0.14]$} & {$[-0.21,0.22]$} & {$[-0.23,0.25]$} \\
$\Delta g_{1}^{Z}$ & 7 & - & - & {$[-0.055,0.071]$} & {$[-0.072,0.085]$} \\
$\lambda_{Z}=\lambda_{\gamma}$ & 8 & {$[-0.011,0.011]$} & {$[-0.014,0.013]$} & {$[-0.013,0.013]$} & {$[-0.015,0.015]$} \\
$\Delta \kappa_{\gamma}$ & 8 & {$[-0.044,0.063]$} & {$[-0.068,0.082]$} & {$[-0.061,0.064]$} & {$[-0.071,0.075]$} \\
$\Delta g_{1}^{Z}$ & 8 & {$[-0.0087,0.024]$} & {$[-0.018,0.028]$} & {$[-0.021,0.024]$} & {$[-0.024,0.027]$} \\
$c_{W W W} / \Lambda^{2}$ & 8 & {$[-2.7,2.7]$} & - & {$[-3.1,3.1]$} & {$[-3.6,3.6]$} \\
$c_{B} / \Lambda^{2}$ & 8 & {$[-14,17]$} & - & {$[-19,20]$} & {$[-22,23]$} \\
$c_{W} / \Lambda^{2}$ & 8 & {$[-2.0,5.7]$} & - & {$[-5.1,5.8]$} & {$[-6.0,6.7]$} \\
\hline
\end{tabular}

Table 15: Expected and observed 95\% CL on the anomalous TGC couplings $\Delta \kappa_{\gamma}, \lambda_{Z}=\lambda_{\gamma}$ and $\Delta g_{1}^{Z}$ measured in the $W^{ \pm} V \rightarrow \ell^{ \pm} \nu q \bar{q} \quad$ final state from the ATLAS [125] and CMS [126] collaborations at $\sqrt{s}=7 \mathrm{TeV}$. The TGC couplings and additional EFT parameters have been extracted at $\sqrt{s}=8 \mathrm{TeV}$ in the $\ell \nu J$ channel from both ATLAS [127] and CMS [128].

tivity over the fully leptonic channels. Analysis extract limits on anomalous TGC and EFT parameters by fitting predictions to the measured transverse momentum distribution of the hadronically decaying boson. Both ATLAS and CMS published limits in for the 7 and $8 \mathrm{TeV}$ data sets. A summary of the available $95 \%$ CL limits in the $\ell \nu q \bar{q}$ channel is given in Table 15 . The 7 TeV ATLAS limits use the LEP scenario [22], while the CMS limits are derived in the HISZ [24, 23] scenario. The $8 \mathrm{TeV}$ limits are for both experiments available in the LEP-constraint scenario and also provided for EFT parametrisation. The achieved precision is better compared to the fully leptonic channel.

Another approach is taken in the CMS measurement of the $W^{ \pm} Z$ and $Z Z$ production cross sections at $\sqrt{s}=8 \mathrm{TeV}$ and a data-set of $18.9 \mathrm{fb}^{-1}$ with two $b$-quark jets from the $Z \rightarrow b \bar{b}$ decay and a leptonically decaying $W^{ \pm}$or $Z$ into a $\ell^{+} \ell^{-}, \nu \bar{\nu}$ or $\ell \nu$ final state [129]. The measured cross sections $\sigma\left(p p \rightarrow W^{ \pm} Z\right)=30.7 \pm 9.3$ (stat) \pm 7.1 (syst) \pm 4.1 (theo) \pm 1.0 (lumi) pb and $\sigma(p p \rightarrow Z Z)=$ $6.5 \pm 1.7$ (stat) \pm 1.0 (syst) \pm 0.9 (theo) \pm 0.2 (lumi) pb are consistent with the calculation at NLO in $\alpha_{s}$ of $\sigma\left(p p \rightarrow W^{ \pm} Z\right)=22.3 \pm 1.1 \mathrm{pb}$ and $\sigma(p p \rightarrow Z Z)=7.7 \pm 0.4 \mathrm{pb}$.

\subsection{Exclusive di-boson production}

With more exclusive selections of single or multiple electroweak boson production, it is possible to enhance the electroweak component of the production process, with superior and sometimes complementary sensitivity to new physics compared to the inclusive process. The first demonstration of this was the observation of the electroweak vector boson fusion process in $Z+2$ jet production by both the ATLAS and CMS experiments [130, 131]. In general, exclusive production is dominated by radiation of vector bosons from incoming quarks accompanied by QCD bremsstrahlung. By selection of certain exclusive regions of the di-jet phase space, typically at very high di-jet invariant mass, the electroweak amplitudes become dominant. The exclusive di-boson production processes discussed in the following are characterised by two vector bosons accompanied by two forward outgoing hadronic systems in the final state. As an example the Feynman diagrams at Born level contributing to the same sign $W^{ \pm} W^{ \pm}$ process are shown in Figure 15 [132]. In this vector bosons scattering process (VBS) the two incoming quarks radiate each an electroweak boson, that subsequently interact and decay. The scattering can involve triple and quartic gauge vertices, hence this process is sensitive to anomalous triple and quartic couplings of vector bosons. Both pure electroweak and QCD amplitudes contribute to the production. The electroweak and QCD amplitudes are typically interfering and cannot be easily isolated from one another via kinematic selections. 


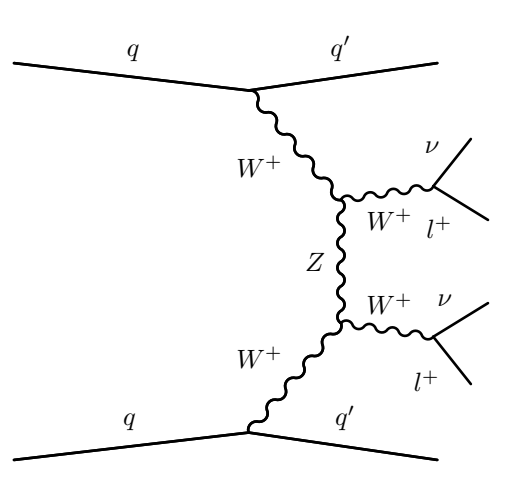

(a)

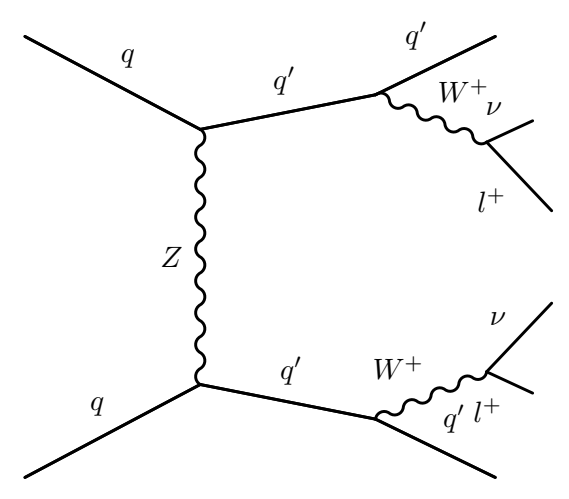

(b)

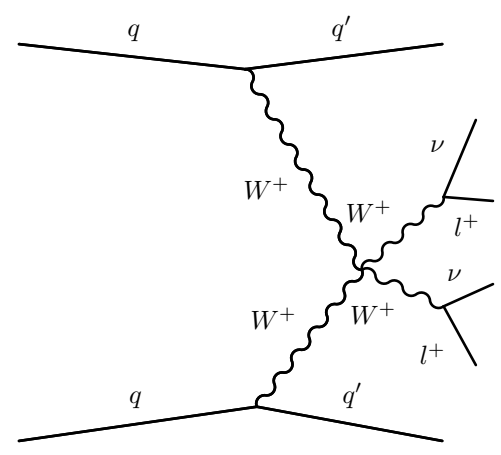

(c)

Figure 15: Representative Feynman diagrams for same-sign $W W$ production in association with two jets from purely electroweak contributions: (left) vector boson fusion, (middle) bremsstrahlung-like, and (right) multiperipheral production [132].

So far exclusive di-boson processes at LHC have been studied in the VBS topology with $W^{ \pm} W^{ \pm}$, $W^{ \pm} Z, Z Z, W^{ \pm} \gamma, Z \gamma, W^{ \pm} V$, and $\gamma \gamma$ initiated $W^{+} W^{-}$production with run-1 and partly run-2 data. A list of studies is available in Table 16. The following sections summarise the results of these studies.

\subsubsection{Exclusive $W^{+} W^{-}$production}

CMS and ATLAS have found evidence for exclusive di-boson production in the process

$$
p p \rightarrow p^{(*)} W^{+} W^{-} p^{(*)} \rightarrow p^{(*)} \mu^{ \pm} e^{\mp} p^{(*)}
$$

The two incoming protons each emit a photon which scatter to produce a $W+W^{-}$pair via eg. a quartic gauge boson vertex, or via $t$ or $u$-channel scattering (see Figure 16). The proton can stay either intact (exclusive production) or dissociate (quasi-exclusive production) into a very forward going hadronic system outside the acceptance of the detector. The signal events in the detector are characterised by two isolated leptons with imbalanced transverse momentum and the absence of underlying event activity otherwise typical for proton-proton hard scatter events.

CMS analysed the combined 7 and $8 \mathrm{TeV}$ data-set [142], while ATLAS looks at exclusive $W^{+} W^{-}$ production with the $8 \mathrm{TeV}$ data-set, also searching for exclusive Higgs production [141]. The event selection requires a mixed flavour $\mu^{ \pm} e^{\mp}$ pair. Same flavour pairs are not feasible in the signal region as the level of background from Drell-Yan produced lepton pairs would be prohibitively high.

The selected leptons are required to have a transverse momentum of $p_{\mathrm{T}}>20 \mathrm{GeV}$, for ATLAS additionally $p_{\mathrm{T}}\left(l_{1}\right)>25 \mathrm{GeV}$ for the leading lepton. The combined transverse momentum must satisfy $p_{\mathrm{T}}(e \mu)>30 \mathrm{GeV}$. Importantly, the primary vertex is required to have zero additional tracks to select exclusive or quasi-exclusive events. A control sample of Drell-Yan events with same flavour leptons $\left(p^{(*)} \mu^{ \pm} \mu^{\mp} p^{(*)}\right.$ or $\left.p^{(*)} e^{ \pm} e^{\mp} p^{(*)}\right)$ is used to study the selection efficiency of the zero-additional track requirement and comparing it with simulated efficiency. Additionally, a cut on the di-lepton invariant mass of $m_{l l}>20 \mathrm{GeV}$ is imposed to remove low mass resonances. Since events with dissociating protons cannot be distinguished from elastic $p p$ scattering events the contribution from single and double dissociative events has to be accounted for. Calculations of these processes are available but do not include rescatter effects that lead to additional activity and thus additional tracks in the events. The relative contribution is deduced from data by comparing same flavour lepton events, i.e. exclusive DrellYan production, to calculations of the cross section (CMS uses LPAIR [143, 144], ATLAS compares to 
Table 16: Summary of exclusive di-boson production studies, grouped by process, experiments, $\sqrt{s}$, and final state. The observed and expected significance $S$ and reference is also given.

\begin{tabular}{c|c|c|c|c|c}
\hline Process & Experiment & $\sqrt{s}[\mathrm{TeV}]$ & Final state & $S[\sigma]$ obs. (exp.) & Reference \\
\hline$p p \rightarrow W^{ \pm} W^{ \pm} q q$ & ATLAS & 8 & $\ell^{ \pm} \nu \ell^{ \pm} \nu q \bar{q}$ & $3.6(2.3)$ & {$[133]$} \\
$p p \rightarrow W^{ \pm} W^{ \pm} q q$ & CMS & 8 & $\ell^{ \pm} \nu \ell^{ \pm} \nu q \bar{q}$ & $2.0(3.1)$ & {$[132]$} \\
$p p \rightarrow W^{ \pm} W^{ \pm} q q$ & CMS & 13 & $\ell^{ \pm} \nu \ell^{ \pm} \nu q \bar{q}$ & $5.5(5.7)$ & {$[134]$} \\
$p p \rightarrow W^{ \pm} \gamma q q$ & CMS & 8 & $\ell \nu \gamma q \bar{q}$ & $2.7(1.5)$ & {$[135]$} \\
$p p \rightarrow Z \gamma q q$ & ATLAS & 8 & $\ell^{+} \ell^{-} \gamma q \bar{q}$ & $2.0(1.8)$ & {$[136]$} \\
$p p \rightarrow Z \gamma q q$ & CMS & 8 & $\ell^{+} \ell^{-} \gamma q \bar{q}$ & $3.0(2.1)$ & {$[137]$} \\
$p p \rightarrow W^{ \pm} Z q q$ & CMS & 13 & $\ell^{+} \ell^{-} \ell \nu q \bar{q}$ & $2.2(2.5)$ & {$[138]$} \\
$p p \rightarrow W^{ \pm} Z q q$ & ATLAS & 13 & $\ell^{+} \ell^{-} \ell \nu q \bar{q}$ & $5.3(3.2)$ & {$[139]$} \\
$p p \rightarrow Z Z q q$ & CMS & 13 & $\ell^{+} \ell^{-} \ell^{+} \ell^{-} q \bar{q}$ & $2.7(1.6)$ & {$[140]$} \\
$\gamma \gamma \rightarrow W^{+} W^{-}$ & ATLAS & 8 & $p \ell^{+} \ell^{-} p$ & 3.0 & {$[141]$} \\
$\gamma \gamma \rightarrow W^{+} W^{-}$ & CMS & 7,8 & $p \ell^{+} \ell^{-} p$ & $3.4(2.8)$ & {$[142]$} \\
$p p \rightarrow W^{ \pm} V q q$ & ATLAS & 8 & $q \bar{q} \ell \nu \gamma q q$ & - & {$[139]$}
\end{tabular}

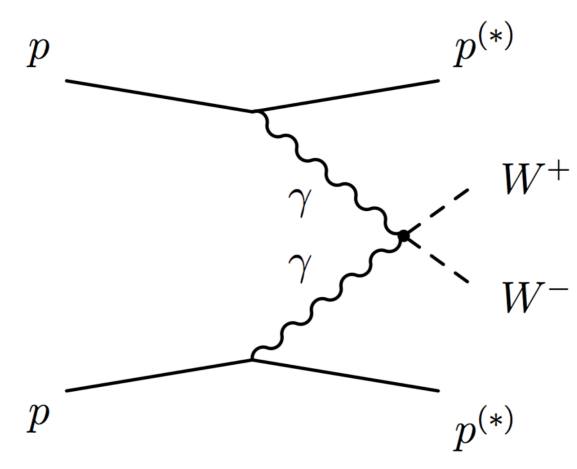

(a)

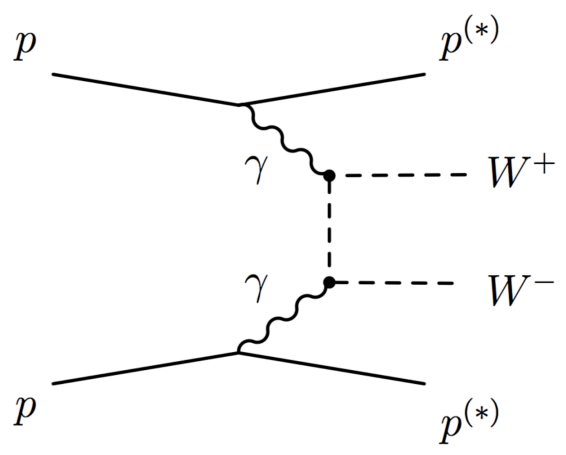

(b)

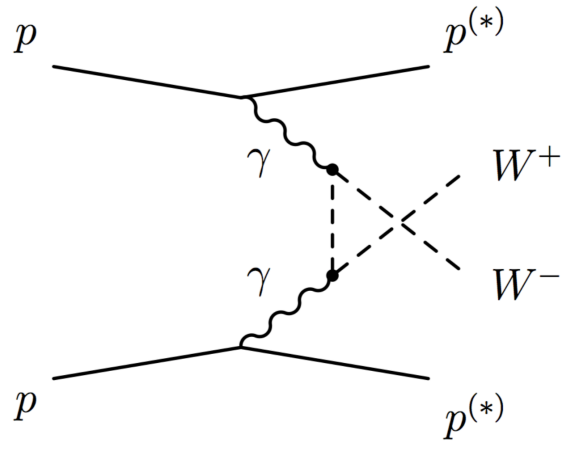

(c)

Figure 16: SM Feynman diagrams contributing to the $\gamma \gamma \rightarrow W^{+} W^{-}$production at leading order, with quartic gauge boson vertex (a), the $s$-channel diagram (b), and the $t$-channel diagram (c) from [142]. The $p^{(*)}$ signifies that the proton can stay intact $(p)$ as in the case for exclusive production, or can dissociate into a hadronic system $\left(p^{*}\right)$ as in the case of quasi-exclusive or diffractive processes. 
Herwig $++[145])$. The proton dissociation component is estimated by ATLAS (CMS) to enhance the signal by a factor of $3.30 \pm 0.23(4.10 \pm 0.43)$ with respect to the calculation in the kinematic region $m_{l l}>160 \mathrm{GeV}$.

The dominant background stems from inclusive di-boson production, primarily $W^{+} W^{-}$, followed by smaller contributions from Drell-Yan processes, $W^{ \pm}+$jets production, and top-quarks production. The $W^{+} W^{-}$background modelling with simulation is verified in control regions that are relaxing the zero-additional track requirement. A leading systematic uncertainty is related to the proton dissociation component for the signal process with an impact of around $10 \%$ and the background determination of around $18 \%$.

After all cuts ATLAS (CMS) observes an excess with a significance of $3.0 \sigma(3.4 \sigma)$ as evidence for exclusive (plus dissociative) $W^{+} W^{-}$production. Figure 17 shows the distributions of the di-lepton $p_{\mathrm{T}}$ after signal selection, and the multiplicity of extra tracks in data compared with simulation. The total cross section in the $8 \mathrm{TeV}$ data if found by CMS to be $10.8_{-4.1}^{+5.1} \mathrm{fb}$, in agreement with a SM prediction of $6.2 \pm 0.5 \mathrm{fb}$. ATLAS also quotes a measurement of the total cross section with $6.0 \pm 2.2$ (stat) \pm 1.4 (sys) $\mathrm{fb}$, also in agreement with a quoted SM prediction of $4.4 \pm 0.3 \mathrm{fb}$.

The quartic boson vertex $\gamma \gamma W^{+} W^{-}$contributing to exclusive $W^{+} W^{-}$production gives this channel sensitivity to anomalous quartic couplings. Both CMS and ATLAS provide 95\% CL limits on anomalous QGC derived from the dilepton $p_{\mathrm{T}}$ distribution with and without applying a cut-off scale, that are comparable in sensitivity. The limits without cut-off scale on the dimension 6 couplings $a_{0}^{W} / \Lambda^{2}$ and $a_{C}^{W} / \Lambda^{2}$ and, in the context of dimension $8 \mathrm{EFT}$, the anomalous couplings $f_{M(0,1,2,3)} / \Lambda^{4}$, are $1.1(4.1) \times$ $10^{-4} \mathrm{GeV}^{-2}$ for $a_{0}^{W} / \Lambda^{2}\left(a_{C}^{W} / \Lambda^{2}\right)$, and range from $2-16 \times 10^{-12} \mathrm{GeV}^{-4}$ for dimension 8 couplings, for models with no form factor in the CMS combined analysis of 7 and $8 \mathrm{TeV}$ data-sets. The ATLAS result using only the $8 \mathrm{TeV}$ data-set has limits of $1.7(6.4) \times 10^{-4} \mathrm{GeV}^{-2}$ for $a_{0}^{W} / \Lambda^{2}\left(a_{C}^{W} / \Lambda^{2}\right)$ and $6-23 \times$ $10^{-11} \mathrm{GeV}^{-4}$ for dimension 8 couplings. The $\gamma \gamma \rightarrow W^{+} W^{-}$process provides the most sensitive channel to constraint $W W \gamma \gamma$ anomalous QGC.
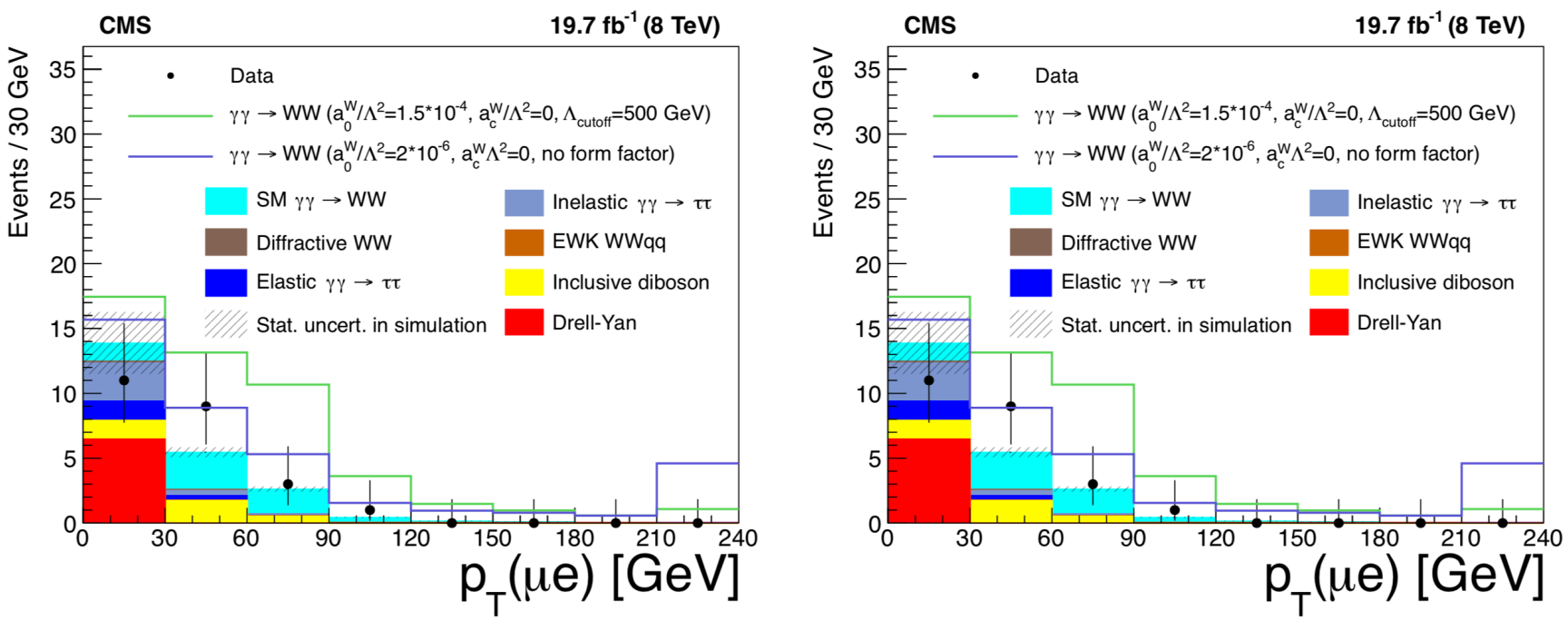

Figure 17: Distributions from the CMS analysis [142] of muon-electron transverse momentum for events with zero associated tracks (left), and extra-tracks multiplicity for events with $p_{\mathrm{T}}\left(\mu^{ \pm} e^{\mp}\right)>30 \mathrm{GeV}$ (right). The data are shown by the points with error bars; the histograms indicate the expected SM signal and backgrounds. 


\subsubsection{The same sign $W W$ process}

The exclusive same-sign $W W$ production, via $q q \rightarrow W W+q^{\prime} q^{\prime} \rightarrow \ell^{ \pm} \ell^{ \pm}+2$ jet $+\mathrm{E}_{\mathrm{T}}^{\text {miss }}$ has a particularly large EW component in the scattering amplitude. The strong production amplitude is suppressed due to absence of gluon-gluon or quark-gluon induced production and has the largest ratio of electroweak to strong production [146]. This channel has also the largest signal-to-background ratio amongst all of the VBS processes and was the first to report evidence for VBS [147, 132, 133].

The diagrams contributing at tree level can be grouped into pure electroweak diagrams with only electroweak vertices and diagrams containing at least one strong interaction vertex. The pure electroweak group of diagrams contain also diagrams with triple and quartic gauge boson interactions (see Figure 15), that constitutes the electroweak vector boson scattering process. Since all diagrams interfere with each other the VBS component cannot be separated, but enhanced through appropriate phase space selection.

The same-sign $W W$ final state has the advantage over other exclusive diboson channels of smaller multi-lepton backgrounds from top quark, Drell-Yan, and $W^{ \pm} Z$ processes due to the same-sign di-lepton requirement. ATLAS has analysed the $8 \mathrm{TeV}$ data-set to establish evidence of electroweakly produced $W^{ \pm} W^{ \pm}$, while CMS has analysed the 8 and $13 \mathrm{TeV}$ data-sets, observing above $5 \sigma$ significance for the process with the $13 \mathrm{TeV}$ data set.

The ATLAS analysis [133] defines three distinct regions to study $W^{ \pm} W^{ \pm}$. Firstly, an "inclusive region" which selects a mixture of electroweak and QCD contributions and defines both as signal, secondly, a "VBS" signal region which is dominated by electroweak production, and thirdly, a region which is optimised to constrain anomalous QGC. The inclusive region requires two same-sign leptons with $p_{\mathrm{T}}>25 \mathrm{GeV}, E_{\mathrm{T}}^{\text {miss }}>40 \mathrm{GeV}$, and at least two jets with $m_{j j}>500 \mathrm{GeV}$ for the two highest $p_{\mathrm{T}}$ jets. The VBS region further requires that the two highest $p_{\mathrm{T}}$ jets are separated in rapidity, $\left|\Delta y_{j j}\right|>2.4$. Finally, the QGC region additionally imposes an additional cut on the transverse mass of the $W^{+} W^{-}$ system to be greater then $400 \mathrm{GeV}$. To reduce Drell-Yan background, events with di-lepton mass less than $20 \mathrm{GeV}$ or di-electrons within $10 \mathrm{GeV}$ of the $Z$ mass are vetoed. Top quark background is reduced by vetoing events with $b$-tagged jets. Finally, the $W Z$ background is reduced by vetoing events with a third lepton, i.e. with an additional muon with $p_{\mathrm{T}}>6 \mathrm{GeV}$ or electron with $p_{\mathrm{T}}>7 \mathrm{GeV}$. Background sources are categorised into prompt backgrounds, i.e. backgrounds with real leptons in the final states from e.g. $W^{ \pm} Z$ production where one lepton is not identified, and non-prompt backgrounds where jets are reconstructed as isolated leptons. Main prompt background sources are estimated from simulation with control regions to verify the correct modelling. Non-prompt background sources are derived with data driven methods. The dominant systematic uncertainty is related to jets, with the total systematic ranging from about $10 \%$ in the signal yield, to about $20 \%$ for the background yield.

After all selection cuts an excess over background is observed in the signal regions and a fiducial cross section is extracted. The di-jet invariant mass in the inclusive region is shown in Figure 18. The composition of events in the inclusive and VBS regions contain about $50 \%$ backgrounds from $W^{ \pm} Z$, $W \gamma$ with photon conversion, and misidentified leptons from jets in $V+$ jet processes. The significance of the signal in the inclusive region is observed (expected) to be $4.5 \sigma(3.1 \sigma)$, and for the VBS region the significance is observed (expected) to be $3.6 \sigma(2.3 \sigma)$. The measured cross sections in these two regions are $2.3 \pm 0.6$ (stat) \pm 0.3 (syst) fb and $1.5 \pm 0.5$ (stat) \pm 0.2 (syst) fb, consistent with SM predictions from Powheg-Box $[63,64,148,149,150,72]$ of $1.52 \pm 0.11 \mathrm{fb}$ and $0.95 \pm 0.06 \mathrm{fb}$, respectively.

The event yield in the QGC region is used to find the 95\% CL limits on anomalous coupling parameters of an effective chiral Lagrangian theory of vector boson scattering [151], calculated by Whizard [152, 153] and using the K-matrix unitarisation scheme [151]: $-0.14<\alpha_{4}<0.15$ and $-0.22<$ $\alpha_{5}<0.22$.

The CMS analyses at $8 \mathrm{TeV}$ [132] and $13 \mathrm{TeV}$ [134] select events with similar criteria as the ATLAS VBS region, eg. in case of the $13 \mathrm{TeV}$ analysis two same-sign leptons with $p_{\mathrm{T}}>25(20) \mathrm{GeV}$ for the 
leading (trailing) lepton, two jets with $m_{j j}>500 \mathrm{GeV}$ and $\left|\Delta \eta_{j j}\right|>2.5$, and $E_{\mathrm{T}}^{\text {miss }}>40 \mathrm{GeV}$. To reduce the background from Drell-Yan production a di-lepton mass of more then $20 \mathrm{GeV}$ is required; for di-electrons events the mass range within $15 \mathrm{GeV}$ of the $\mathrm{Z}$ mass is excluded. Events with top quarks are vetoed with $b$-jet tag using secondary vertex information and multi-variate techniques. To reduce background from $W^{ \pm} Z$ events a third lepton veto for $p_{\mathrm{T}}>10 \mathrm{GeV}$ and looser reconstruction requirements is imposed.

After all selection cuts a total of 201 events are selected in data with a total background expectation of $138 \pm 13$ establishing an observation at the $5.5 \sigma$ level with $5.7 \sigma$ expected. In Figure 18 the dijet invariant mass spectrum is shown, comparing data to background and signal expectation. The background is mostly from misidentified leptons. The observed fiducial cross section is $3.83 \pm 0.66$ (stat) \pm 0.35 (syst) $\mathrm{fb}$ and is in good agreement with the expected cross section of $4.25 \pm 0.27 \mathrm{fb}$ at LO.

The $m_{\ell \ell}$ distribution is used to obtain $95 \%$ CL limits on dimension 8 EFT couplings $f_{S,(0,1)}$, $f_{M,(0,1,6,7)}$, and $f_{T,(0,1,2)}[154]$. The limits are improved by a factor of about seven compared to the $8 \mathrm{TeV}$ CMS analysis [132]. Due to the different frameworks used by CMS and ATLAS a direct comparison of limits on EFT parameters between experiments is up to now not possible for this channel.

\subsubsection{Exclusive $W^{ \pm} Z$ production}

The VBS process $p p \rightarrow W^{ \pm} Z q q$ has, similar to the $p p \rightarrow W^{ \pm} W^{ \pm} q q$ process, a large electroweak component, and is sensitive to the $W^{ \pm} W^{ \pm} Z Z$ quartic boson vertex. It was the second VBS process after $p p \rightarrow W^{ \pm} W^{ \pm} q q$ to be observed at the LHC. Because of only one neutrino in the final state the channel allows to measure the polarisation of the vector bosons by constraining the $W^{ \pm}$mass. While the opposite sign $W^{+} W^{-}$scattering process is dominated by the strong scattering amplitude, the $W^{ \pm} Z$ channel offers a reasonable production cross section and a large weak scattering amplitude. Both ATLAS and CMS have analysed the $13 \mathrm{TeV}$ data-set and provide measurements of fiducial cross sections. The exclusive $W^{ \pm} Z$ production is kinematically similar to the $W^{ \pm} W^{ \pm}$exclusive process, but the electroweak component of the cross section is smaller.

The CMS analysis [138] looks at the $13 \mathrm{TeV}$ data-set. Signal events are required to have three isolated leptons $(\ell=e, \mu)$, of which two must form a $Z$ candidate with the di-lepton invariant mass satisfying $\left|m_{\ell \ell}-\mathrm{m}_{Z}\right|<15 \mathrm{GeV}$. Events with additional leptons are vetoed. Further, a veto on $b$-tagged jets is imposed to reduce the background from top quark events. The tri-lepton invariant mass must be above $100 \mathrm{GeV}$ to reduce the background from $Z$ bosons with final state photon radiation. The $E_{\mathrm{T}}^{\text {miss }}$ is required to be at least $30 \mathrm{GeV}$. At least two jets are required with $m_{j j}>500 \mathrm{GeV}$ and $\left|\Delta \eta_{j j}\right|>2.5$. Control regions outside the signal region are used to constrain the background normalisation from $\mathrm{QCD} W^{ \pm} Z$ production. Prompt and non-prompt background sources are treated separately. Prompt background, eg. $Z Z$, are estimated with simulation, while non-prompt backgrounds, mainly top-quark pair and $Z+$ jet production, are estimated from control regions in data. The main systematic uncertainty is related to the jet energy scale with an impact of $7 \%$ on the signal. For the extraction of the fiducial cross section, designed to enhance the electroweak amplitude of the process, a combined maximum likelihood fit to the leptonic decay channels $(e e e, e e \mu, \mu \mu e, \mu \mu \mu)$ under consideration of statistical and systematic uncertainties and correlations is performed. A fiducial cross section of $3.18_{-0.52}^{+0.57}$ (stat) ${ }_{-0.36}^{+0.43}$ (syst) $\mathrm{fb}$ is found, in agreement with the prediction of $3.27_{-0.32}^{+0.39}$ (scale) $\pm 0.15(\mathrm{PDF}) \mathrm{fb}$. The purely electroweak component in this fiducial region is about $40 \%$ of the cross section, and the interference between strong and electroweak amplitudes is estimated to yield less then 1\% in this region. The electroweak fraction of the cross section increases for higher di-jet invariant mass $m_{j j}$ and separation in rapidity $\Delta \eta_{j j}$ as seen in Figure 19. The pure electroweak signal is extracted from this distribution with a binned likelihood fit, and the signal strength found to be $\mu_{E W}=0.82_{-0.43}^{+0.51}$ corresponding to an observed significance for electroweak $W^{ \pm} Z$ production of $2.3 \sigma$ and $2.5 \sigma$ expected.

CMS also extracts limits on dimension 8 EFT couplings $f_{S,(0,1)}, f_{M,(0,1)}$, and $f_{T,(0,1,2)}[154]$ with 

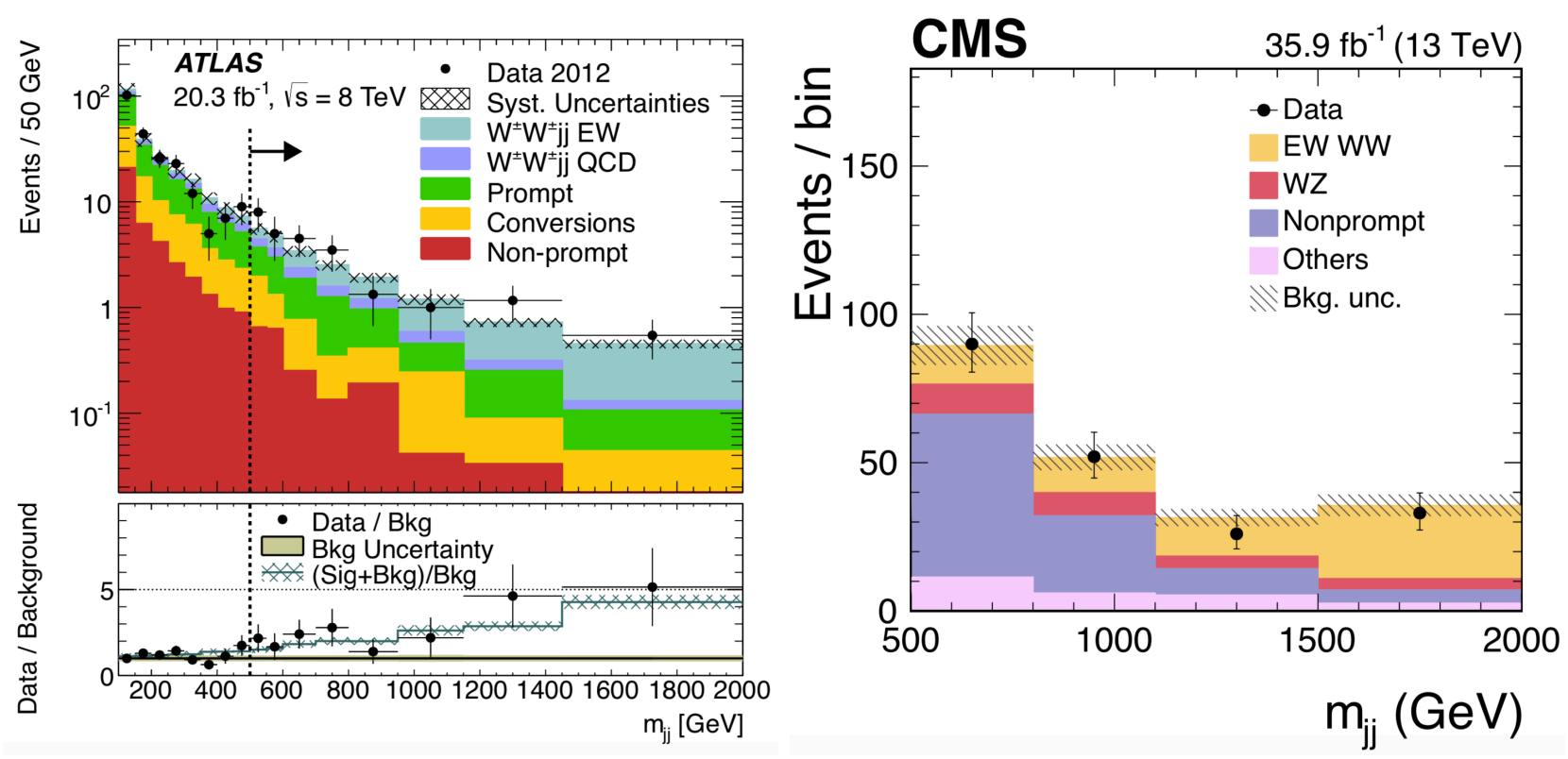

Figure 18: (a) the di-jet invariant mass distribution of $W^{ \pm} W^{ \pm} j j$ candidates selected by ATLAS [147]. The inclusive signal region is indicated by the arrow. (b) the di-jet invariant mass distribution of $W^{ \pm} W^{ \pm} j j$ candidates selected by CMS [132].

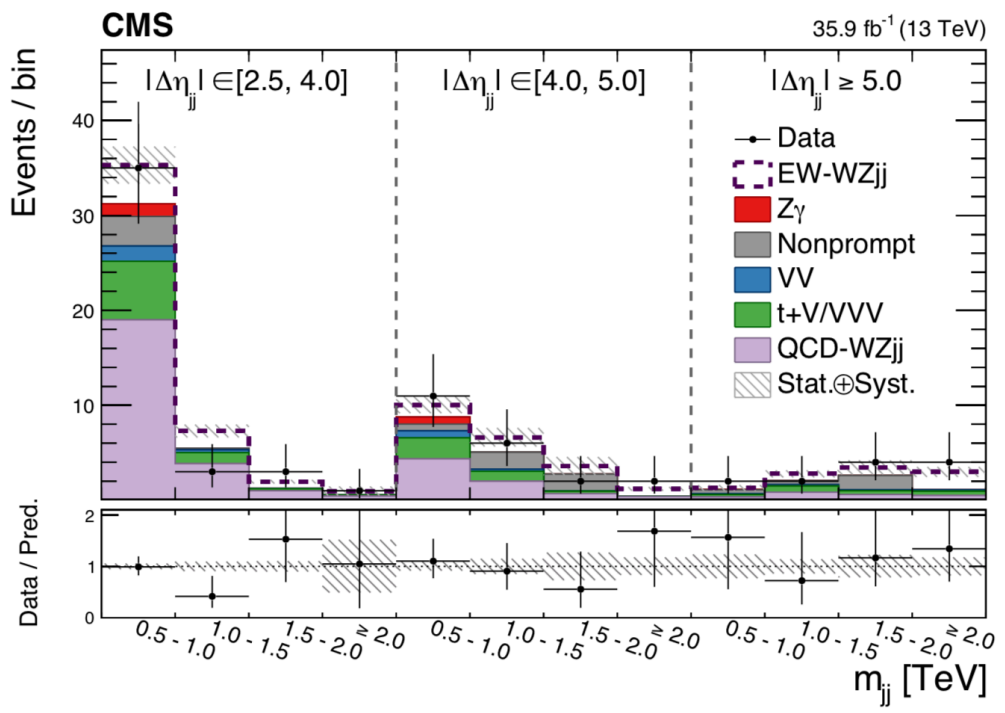

Figure 19: The di-jet invariant mass distribution for different ranges of $\left|\Delta \eta_{j j}\right|$ from the $13 \mathrm{TeV}$ VBS $W^{ \pm} Z$ CMS analysis [138]. The pure electroweak component of the VBS $W^{ \pm} Z$ process is shown as the dashed line on top of stacked histogram representing the background sources. The hatched band indicates the total uncertainty. The ratio of data over prediction is given in the bottom part. 
the transverse mass distribution of the $W^{ \pm} Z$ system. Compared to the $W^{ \pm} W^{ \pm}$channel the precision is less impressive due to the reduced electroweak component, however the $W^{ \pm} Z$ process provides an independent measurement of these operators.

The 13 TeV ATLAS analysis [139] follows a similar approach to CMS. The cut on di-lepton invariant mass of the $Z$ candidate is tighter with $\left|m_{\ell \ell}-m_{Z}\right|<10 \mathrm{GeV}$. However, no requirement on the rapidity difference of the two tagging jets are imposed. The final VBS region also requires $m_{j j}>500 \mathrm{GeV}$. A $b$-jet veto reduces top quark related backgrounds. The dominant background source of QCD induced $W^{ \pm} Z$ production is modelled with simulation, with the normalisation obtained from data control regions. The sub-leading prompt background contributions from $Z Z$ and $t \bar{t}+Z / W$ processes are modelled in the same manner. Non-prompt backgrounds from eg. $Z+$ jets, $Z \gamma, t \bar{t}$ are determined with data driven methods. The dominant systematic uncertainty on the fiducial cross section is related to the jet reconstruction. The theory modelling uncertainty is about $7 \%$. The signal is extracted using a multivariate technique, exploiting the kinematic differences of the electroweakly and strongly produced $W^{ \pm} Z$ events, as well as other backgrounds. To reach optimum discrimination power between these three categories 15 kinematic variables are fed into a boosted decision tree and trained on simulated events. After all selection cuts 161 events are observed in data and $167 \pm 11$ are expected. The extracted signal composition is $44 \pm 11$ electroweakly produced $W^{ \pm} Z$ events, $91 \pm 10$ QCD produced $W^{ \pm} Z$ events and the rest from other background sources. The measured $p p \rightarrow W^{ \pm} Z q q$ fiducial cross section is $1.68 \pm 0.25 \mathrm{fb}$. This can be compared to a prediction from SHERPA combining QCD induced and electroweak production of $2.15 \pm 0.01$ (stat) $\pm 0.05(\mathrm{PDF})_{-0.44}^{+0.65}$ (scale). The signal strength of the electroweak production is found to be $\mu=1.77_{-0.43}^{+0.49}$ corresponding to a significance of $5.3 \sigma$, whereas $3.2 \sigma$ are expected.

\subsubsection{Exclusive $Z Z$ production}

The $p p \rightarrow Z Z q q$ process has a lower production cross section compared to $p p \rightarrow W^{ \pm} W^{ \pm} q q$ or $p p \rightarrow$ $W^{ \pm} Z q q$, but has the advantage that the decay products are all reconstructed and the full event kinematic information is accessible, including the energy of the scatter event $\hat{s}$. Also the process has very few and reducible backgrounds, mainly from misidentified leptons. The process receives contribution from electroweak and QCD induced production, example Feynman diagrams are shown in Figure 20 taken from [140].

At the time of writing only CMS has published a measurement of the exclusive $Z Z$ production at $\sqrt{s}=13 \mathrm{TeV}[140]$ with $35.9 \mathrm{fb}^{-1}$ of data. The signal is selected by requiring events with two $Z$ candidates from opposite sign same flavour ( $e$ or $\mu$ ) lepton pairs, and the mass of the $Z$ candidates in the range of 40 to $120 \mathrm{GeV}$. For the electroweak production of $p p \rightarrow Z Z q q$ at least two jets are required with the di-jet invariant mass larger than $100 \mathrm{GeV}$.

The main background source to the electroweak process is the QCD induced $Z Z$ production. The QCD induced production and the electroweak signal are separated with multivariate techniques, i.e. a boosted decision tree (BDT) that is trained with simulated QCD and electroweak samples and has in total 36 input variables. The method is validated in a QCD enriched control region defined by events with $m_{j j}<400 \mathrm{GeV}$ or $\left|\Delta \eta_{j j}\right|<2.4$. Important systematic uncertainties are renormalisation and factorisation scale uncertainties and jet energy scale. The output distributions of the BDT (see Figure 21) are used to extract the significance of the electroweak signal by fitting the signal and background components in the signal and control regions. The electroweak signal strength is found to be $\mu=1.39_{-0.65}^{+0.86}$ corresponding to a significance of $2.7 \sigma$ observed (1.6 $\sigma$ expected).

The $p p \rightarrow Z Z q q$ process probes the charged coupling parameters $f_{T,(0,1,2)}$ also accessible with $p p \rightarrow$ $W^{ \pm} W^{ \pm} q q$ and $p p \rightarrow W^{ \pm} Z q q$, but has also sensitivity to the neutral couplings $f_{T,(8,9)}$. Consequently, CMS reports limits on dimension 8 EFT parameters $f_{T,(0,1,2,8,9)}$ [154], using the di-boson invariant mass distribution $m_{Z Z}$ that provides the highest sensitivity to anomalous couplings. The $95 \% \mathrm{CL}$ limits for the neutral couplings are $-0.84 \mathrm{TeV}^{-4}<f_{T 8} / \Lambda^{4}<0.84 \mathrm{TeV}^{-4},-1.8 \mathrm{TeV}^{-4}<f_{T 9} / \Lambda^{4}<1.8 \mathrm{TeV}^{-4}$. 

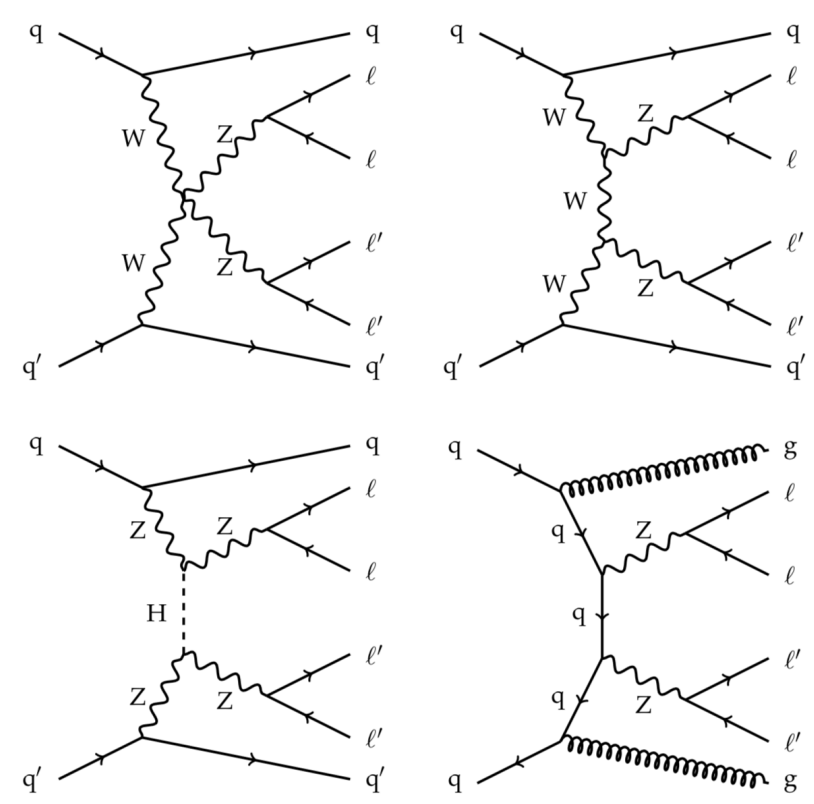

Figure 20: Representative Feynman diagrams contributing to the process $p p \rightarrow Z Z q q$ from [140]. The top row and bottom left represent electroweak induced production, the bottom right is an example of QCD induced production.
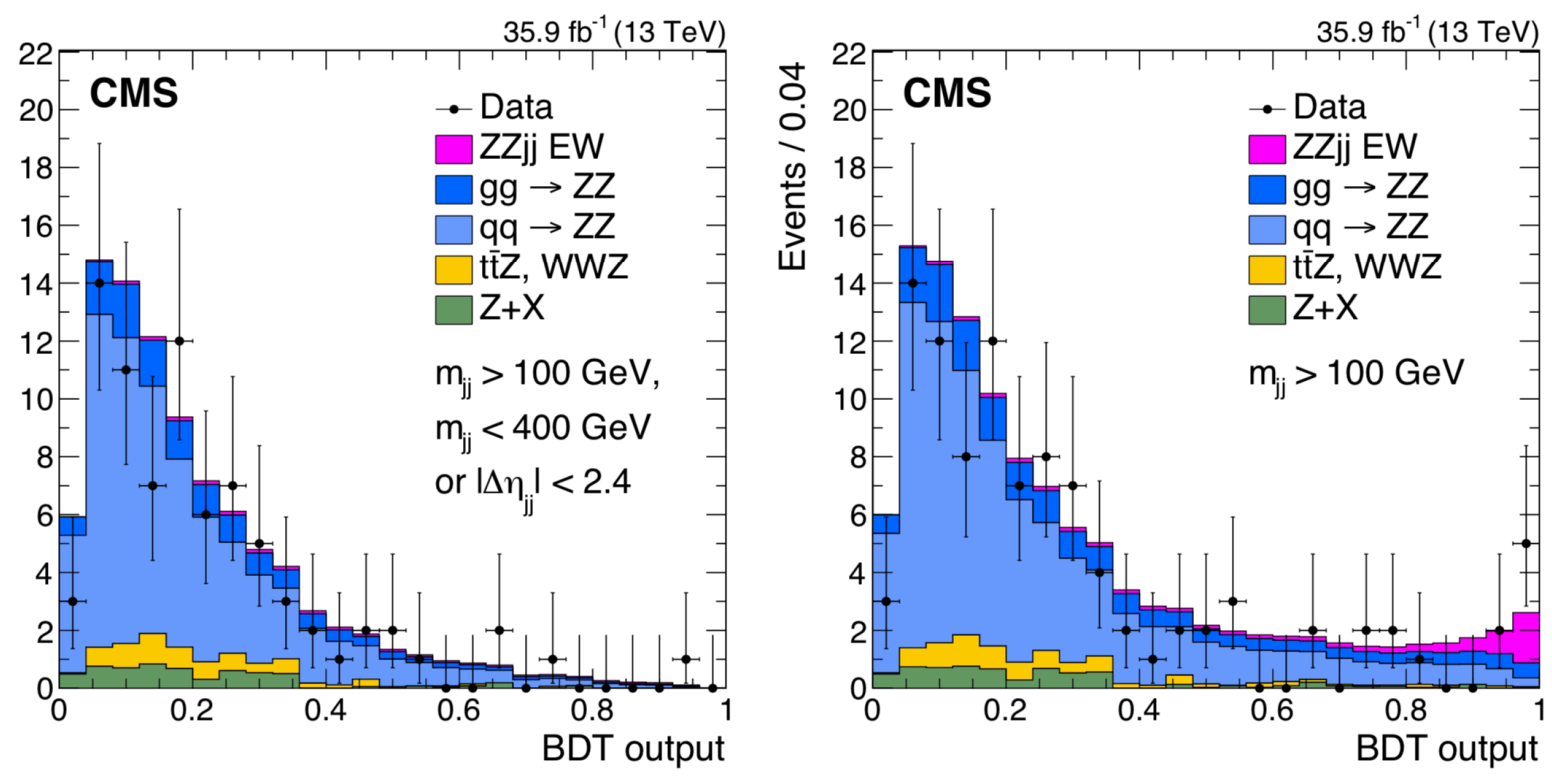

Figure 21: The BDT output distributions for a QCD enriched control region defined by events with $m_{j j}<400 \mathrm{GeV}$ or $\left|\Delta \eta_{j j}\right|<2.4$, and for the signal region (right) from [140]. 


\subsubsection{Exclusive $V \gamma$ production}

The process $p p \rightarrow W^{ \pm} \gamma q q$ has the largest production cross section among the VBS processes which makes it a promising channel to study. So far only a study by CMS at $\sqrt{s}=8 \mathrm{TeV}$ is available in this channel [135]. The $p p \rightarrow Z \gamma q q$ process has a smaller cross section but provides a cleaner signature with two charged leptons and the ability to use the mass constrain from the $Z$ decay. Both channel offer complementary sensitivity to different dimension 8 operators of anomalous quartic gauge couplings. Studies are available from CMS and ATLAS at $\sqrt{s}=8 \mathrm{TeV}$ in the $\ell^{+} \ell^{-} \gamma q \bar{q}[136,137]$ and $\nu \nu \gamma q \bar{q}$ [136] final state. Since QCD induced and pure electroweak VBS amplitudes interfere, fiducial regions are optimised for QCD+electroweak or electroweak induced production. The interference effect ranges from $5 \%\left(p p \rightarrow W^{ \pm} \gamma q q\right)$ to $10 \%(p p \rightarrow Z \gamma q q)$ in the electroweak fiducial region.

To select the signal for $p p \rightarrow W^{ \pm} \gamma q q$ an isolated muon (electron) with $p_{\mathrm{T}}>25(30) \mathrm{GeV}$ within $|\eta|<2.1(2.4)$, a photon candidate with $p_{\mathrm{T}}>22 \mathrm{GeV}$ and $|\eta|<1.44$, and two jets with $m_{j j}>200 \mathrm{GeV}$ to suppress $W V \gamma$ and a minimum separation of $\Delta R>0.5$ between these reconstructed objects are required. A veto on events with additional leptons is imposed. Additional cuts on transverse mass of the $W^{ \pm}$candidate and a missing momentum of at least $35 \mathrm{GeV}$ help to suppress the QCD background. For $p p \rightarrow Z \gamma q q$ two opposite sign same flavour leptons with the invariant di-lepton mass compatible with a $Z$ candidate and two jets with $m_{j j}>150 \mathrm{GeV}$ are required. The main background sources to the electroweak process are QCD induced $p p \rightarrow W^{ \pm} \gamma q q$ production. The background is modelled with simulation, but the normalisation is constrained with a QCD enriched control region. Another important background is $V+$ jet production where the jet is misidentified as an isolated photon. This background is estimated from data with dedicated control region to assess the probability of a jet being misidentified as a photon, taking into account the different of control and signal region in terms of kinematic acceptance. Other background sources are small and generally estimated from simulation.

After all selection cuts CMS uses the binned $m_{j j}$ distribution to extract the electroweak signal strength in the $p p \rightarrow W^{ \pm} \gamma q q$ in a kinematic region above $m_{j j}>700 \mathrm{GeV}$. A signal strength of $\mu=1.78_{-0.76}^{+0.99}$ is observed corresponding to a significance of $2.7 \sigma$ (expected $1.5 \sigma$ ), in agreement with the theoretical prediction. The transverse momentum spectrum of the $W^{ \pm}$candidate is used to set limits on dimension 8 EFT couplings and 95\% CL limits on the parameters $f_{M,(0,1,2,3,4,5,6,7)} / \Lambda^{4}, f_{T,(0,1,2,5,6,7)} / \Lambda^{4}$ are reported. A subset of these couplings has been accessed with other multiboson processes discussed in the sections above, but this channel provides unique access to constrain the parameters $-40 \mathrm{TeV}^{-4}<$ $f_{M, 4} / \Lambda^{4}<40 \mathrm{TeV}^{-4},-3.8 \mathrm{TeV}^{-4}<f_{T, 5} / \Lambda^{4}<3.8 \mathrm{TeV}^{-4},-2.8 \mathrm{TeV}^{-4}<f_{T, 6} / \Lambda^{4}<3.0 \mathrm{TeV}^{-4}$, and $-7.3 \mathrm{TeV}^{-4}<f_{T, 7} / \Lambda^{4}<7.7 \mathrm{TeV}^{-4}$.

In the $p p \rightarrow Z \gamma q q$ channel the CMS analysis defines a fiducial phase space to extract the electroweak production signal in the high di-jet mass region with $m_{j j}>400 \mathrm{GeV}$. The expected electroweak signal component of $p p \rightarrow Z \gamma q q$ is estimated to be $26 \%$. A signal strength of $\mu=1.5_{-0.6}^{+0.9}$ is found, corresponding to a significance of $3 \sigma$ (expected $2.1 \sigma$ ) and in agreement with a LO prediction. ATLAS extracts the signal strength from a likelihood fit over the "centrality" [136] of the $Z \gamma$ two-body system after applying cuts to enhance the electroweak component and obtains a significance of $2 \sigma$. CMS extracts limits for dimension $8 \mathrm{EFT}$ operators from the di-boson invariant mass distribution, $m_{Z \gamma}$. Limit on the parameter $f_{M,(0,1,2,3)} / \Lambda^{4}, f_{T,(0,1,2,3,8,9)} / \Lambda^{4}$ are given. The ATLAS analysis combines the $\ell^{+} \ell^{-} \gamma q \bar{q}$ and $\nu \nu \gamma q \bar{q}$ final states and optimises the phase space with more stringent cuts (eg. $m_{j j}>600 \mathrm{GeV}$ ) to gain sensitivity. The $95 \%$ CL limits on the parameter $f_{M,(0,1,2,3)} / \Lambda^{4}, f_{T,(0,8,9)} / \Lambda^{4}$ are obtained, and show a similar sensitivity compared to CMS results in this channel.

\subsection{Tri-boson production}

Processes with three (on-shell) vector bosons in the final state have the lowest production cross section (of the order of $1 \mathrm{pb}$ ) amongst all studied multi-boson processes, typically one to two orders of magnitude 
Table 17: Summary of exclusive tri-boson production studies, grouped by process, experiments, $\sqrt{s}$, and final state. The observed and expected significance $S$ and reference are provided in the two last columns.

\begin{tabular}{c|c|c|c|c|c}
\hline Process & Experiment & $\sqrt{s}[\mathrm{TeV}]$ & Final state & $S[\sigma]$ obs. (exp.) & Reference \\
\hline$W^{ \pm} \gamma \gamma$ & ATLAS & 8 & $\ell \nu \gamma \gamma,(\ell=e, \mu)$ & $>3$ & {$[155]$} \\
$W^{ \pm} \gamma \gamma$ & CMS & 8 & $\ell \nu \gamma \gamma,(\ell=e, \mu)$ & 2.6 & {$[9]$} \\
$Z \gamma \gamma$ & ATLAS & 8 & $\ell \ell \gamma \gamma,(\ell=e, \mu)$ & 6.3 & {$[8]$} \\
$Z \gamma \gamma$ & CMS & 8 & $\ell \ell \gamma \gamma,(\ell=e, \mu)$ & 5.9 & {$[9]$} \\
$W^{ \pm} W^{ \pm} \gamma$ & ATLAS & 8 & $e \nu \mu \nu \gamma,(\ell=e, \mu)$ & $1.4(1.6)$ & {$[156]$} \\
$W^{ \pm} V \gamma$ & ATLAS & 8 & $\ell \nu q \bar{q} \gamma,(\ell=e, \mu)$ & - & {$[156]$} \\
$W^{ \pm} V \gamma$ & CMS & 8 & $\ell \nu q \bar{q} \gamma,(\ell=e, \mu)$ & - & {$[157]$} \\
$W^{ \pm} W^{ \pm} W^{\mp}$ & ATLAS & 8 & $\ell \nu \ell \nu q \bar{q}, \ell \nu \ell \nu \ell \nu,(\ell=e, \mu)$ & - & {$[158]$}
\end{tabular}

lower than the experimentally comprehensively covered inclusive processes with two vector bosons. With the full run-1 and run-2 data-sets available, the analysis are generally statistically limited. First evidence was reported in final states involving one massive vector boson and two photons, $V \gamma \gamma$, with run-1 data $[9,155]$. Other channels with two or three massive vector bosons have been investigated, but have a lower cross section compared to $V \gamma \gamma$ and become accessible with run-2 data at $\sqrt{s}=13 \mathrm{TeV}$. Table 17 summarises the currently available inclusive tri-boson studies. Although the analysis are statistically limited, the direct contribution of the four boson vertex allows to set limits on anomalous QGC comparable in precision to the exclusive processes.

\subsubsection{The $V \gamma \gamma$ process}

The $V \gamma \gamma$ process receives contributions from single $W^{ \pm}$production with final or initial state radiation of a proton or the charged lepton of the $V$ decay, and from triple or quartic gauge vertices. Representative Feynman diagrams of this process for $V=Z$ are shown in Figure 22. To reduce the FSR and ISR contribution cuts on the transverse momentum on the photon candidates and the separation between leptons and photons are imposed. CMS and ATLAS have published results with the $8 \mathrm{TeV}$ data-set for the $V \gamma \gamma$ process with $V=W^{ \pm}, Z$ and the leptonic decay channel.

The $W^{ \pm} \gamma \gamma$ ATLAS analysis selects signal events requiring two isolated photons and one isolated electron or muon with $p_{\mathrm{T}}>20 \mathrm{GeV}$. The transverse mass of the $W^{ \pm}$candidate is required to be above $40 \mathrm{GeV}$. To suppress background from $Z \gamma$ events, where one electron from the $Z \rightarrow e^{+} e^{-}$decay is misidentified as a photon, the transverse momentum of the $e \gamma \gamma$ system must be greater than $30 \mathrm{GeV}$, and the invariant mass of $e \gamma(\gamma)$ system must be outside the $Z$ mass range. After selection cuts 47 events are observed in the electron channel with a background expectation of $30.2 \pm 5.0$ (stat) \pm 5.4 (syst), and 110 events in the muon channel with a background only expectation of $52.1 \pm 8.9$ (stat) \pm 8.4 (syst). The excess of events corresponds to a signal significance above the $3 \sigma$ level. Figure 23 shows the diboson invariant mass distribution for the electron and muon channels, comparing data and prediction for background and signal, where the signal excess is demonstrated.

The fiducial production cross section is estimated for both the inclusive case and an exclusive selection with a jet veto for jets with $p_{\mathrm{T}}>30 \mathrm{GeV}$. The inclusive cross section is measured as $6.1_{-1.0}^{+1.1}$ (stat) \pm 1.2 (syst) \pm 0.2 (lumi.) fb, and is $1.9 \sigma$ above the NLO prediction from MCFM of $2.90 \pm 0.16$ $\mathrm{fb}$. The exclusive cross section is determined as $2.9_{-0.7}^{+0.8}(\text { stat })_{-0.9}^{+1.0}$ (syst) \pm 0.1 (lumi) fb and agrees with the MCFM prediction of $1.88 \pm 0.20 \mathrm{fb}$. The better agreement in the exclusive fiducial volume is likely due to missing higher order correction, and a similar effect is observed in the $W \gamma / Z \gamma$ analysis, where the comparison of the inclusive cross section to NNLO calculation showed a much better agreement. The 


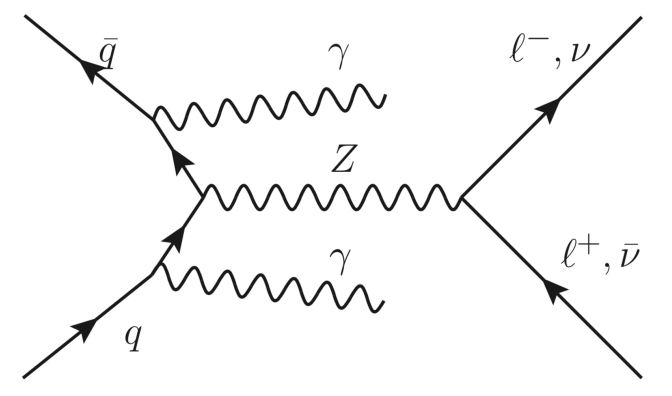

(a)

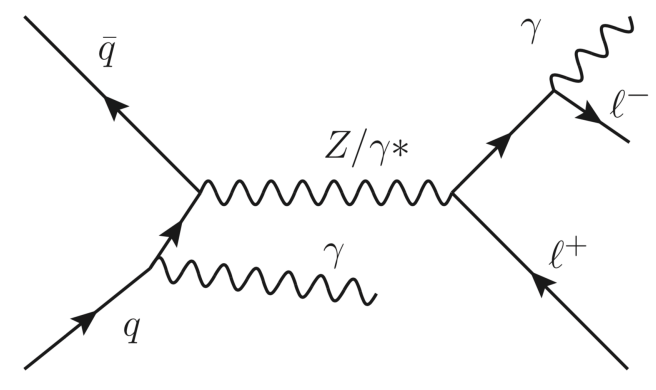

(c)

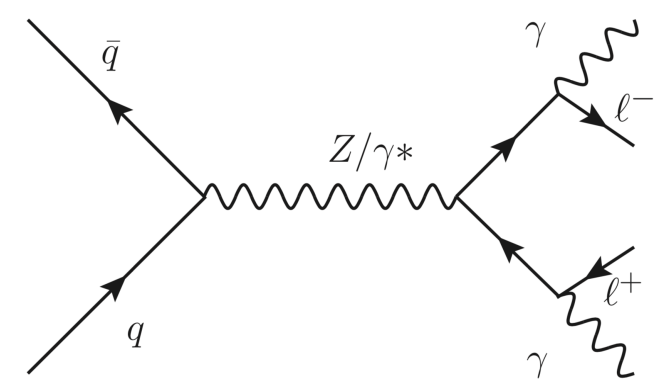

(b)

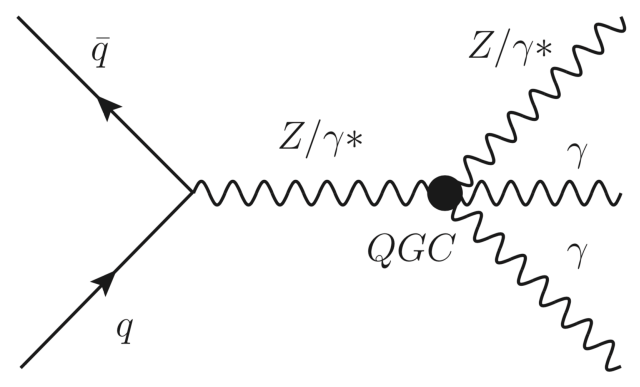

(d)

Figure 22: Representative Feynman production diagrams for the process $Z \gamma \gamma$ from [8], a) initial state radiation, b) final state radiation, c) mixed initial and final state radiation, and d) quartic gauge boson vertex.
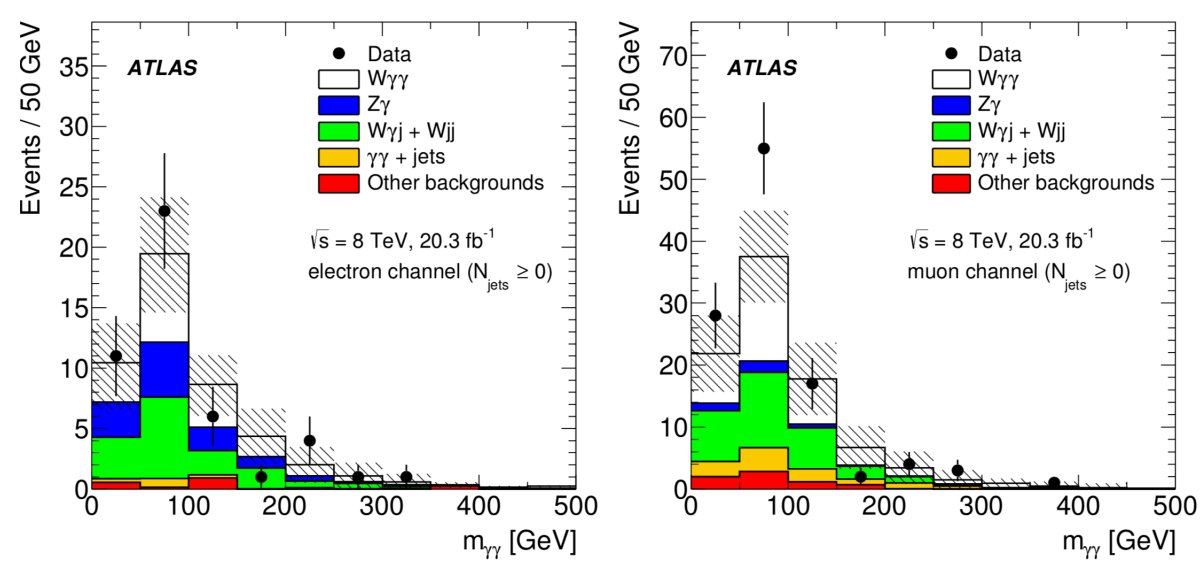

(a)

Figure 23: Diphoton invariant mass spectrum in the electron (left) and muon (right) channel of the ATLAS $W^{ \pm} \gamma \gamma 8 \mathrm{TeV}$ analysis [155]. Data is compared to expected signal and background from simulation, with the hashed area showing the combined statistical and systematic uncertainty. 
dominant systematic uncertainties are related to the data-driven background determination methods. The diphoton invariant mass spectrum above $m_{\gamma \gamma}>300 \mathrm{GeV}$ is sensitive to anomalous QGC and ATLAS sets $95 \%$ CL limits on the dimension 8 EFT parameters $f_{T, 0} / \Lambda^{4}, f_{M, 2,3} / \Lambda^{4}$, which can be compared to the limits obtained in the $W^{ \pm} W^{ \pm}$channel.

CMS follows a similar strategy in the $W^{ \pm} \gamma \gamma$ channel [9]. The analysis requires one isolated electron (muon) with $p_{\mathrm{T}}>30(25) \mathrm{GeV}$ and two isolated photon with $p_{\mathrm{T}}>25 \mathrm{GeV}$, a transverse mass above 40 $\mathrm{GeV}$, and a minimum separation of $\Delta R>0.4$ between the reconstructed objects. Also events with the invariant mass of $e \gamma(\gamma)$ system in the $Z$ mass range are vetoed. The analysis observes a fiducial cross section of $4.9 \pm 1.4$ (stat) \pm 1.6 (syst) \pm 0.1 (lumi) fb corresponding to a signal significance of $2.6 \sigma$, and in good agreement with a NLO prediction of $4.8 \pm 0.5 \mathrm{fb}$. Again, the dominant uncertainty is associated to the photon misidentification rate from QCD jets. Also CMS sets stringent 95\% CL limits on the dimension 8 EFT parameters $f_{T, 0} / \Lambda^{4}, f_{M, 2,3} / \Lambda^{4}$.

CMS studies the $Z \gamma \gamma$ channel [9] in the same $8 \mathrm{TeV}$ data sample. The lepton selection cuts are less stringent with requiring $p_{\mathrm{T}}>20(10) \mathrm{GeV}$ for electrons (muons) compared to the $W^{ \pm} \gamma \gamma$ channel. Two opposite same flavour leptons with an invariant mass greater than $40 \mathrm{GeV}$ and two photons with $p_{\mathrm{T}}>15 \mathrm{GeV}$ are required. A fiducial cross section of $12.7 \pm 1.4$ (stat) \pm 1.8 (syst) \pm 0.3 (lumi) fb is observed in agreement with the NLO prediction of $13.0 \pm 1.5 \mathrm{fb}$. The slightly lower relative systematic uncertainty reflects the reduced systematic uncertainty contribution from the jet related background determination. The signal significance is correspondingly higher with $5.9 \sigma$. EFT coupling parameter limits are not reported.

The same channel $Z \gamma \gamma$ is also studied by ATLAS in the $8 \mathrm{TeV}$ data set [8]. The selection of signal events requires two opposite sign same flavour leptons ( $e$ or $\mu$ ) with invariant mass above $40 \mathrm{GeV}$ and at least two isolated photons with $p_{\mathrm{T}}>15 \mathrm{GeV}$ and a minimum distance of $\Delta R>0.4$. Additionally, $\nu \nu \gamma \gamma$ events are considered, requiring $E_{\mathrm{T}}^{\text {miss }}>110 \mathrm{GeV}$, for the photons $p_{\mathrm{T}}>22 \mathrm{GeV}$, and that the photon system and MET are back to back in the transverse plane. ATLAS observes a fiducial cross section of $5.07_{-0.68}^{+0.73}(\text { stat })_{-0.38}^{+0.41}$ (syst) \pm 0.10 (lumi) fb in the $\ell \ell \gamma \gamma$ final state corresponding to a significance of $6.0 \sigma$, and $2.5_{-0.9}^{+1.0}$ (stat) \pm 1.1 (syst) \pm 0.1 (lumi) fb in the $\nu \nu \gamma \gamma$ channel. The systematic uncertainty on the $\nu \nu \gamma \gamma$ measurement is driven by the background determination, while in the case of $\ell \ell \gamma \gamma$ reconstruction and background systematics are of similar importance. A kinematic region sensitive to anomalous QGC is defined (zero-jet, $m_{\gamma \gamma}>300(200) \mathrm{GeV}$ for the $\nu \nu \gamma \gamma(\ell \ell \gamma \gamma)$ channel) to set $95 \% \mathrm{CL}$ limits on dimension 8 EFT parameters $f_{T, 0,5,9} / \Lambda^{4}$ and $f_{M, 2,3} / \Lambda^{4}$ using the combined statistical power of both channels, reaching comparable sensitivity to the $W^{ \pm} \gamma \gamma$ channel.

\subsubsection{The $W^{ \pm} V \gamma$ process}

The tri-boson final state $W^{ \pm} V \gamma$ with $V=W^{ \pm}, Z$ is more challenging as the final state with two massive vector bosons has a lower cross section compared to $V \gamma \gamma \cdot W^{ \pm} V \gamma$ has been searched for in the fully leptonic and semi-leptonic decay channel. Searches with the $\sqrt{s}=8 \mathrm{TeV}$ data-set by ATLAS [156] and CMS [157] are available.

The CMS analysis searches in the semi-leptonic decay channel, $\ell \nu q q \gamma$, for $W^{ \pm} V \gamma$ production. The hadronically decaying $V$ offers a higher branching fraction compared to the fully leptonic channel, but does not allow to distinguish between $W^{ \pm}$and $Z$ decays because of the limited resolution on the invariant mass of the hadronic system $(\mathrm{O}(10 \%))$, thus the signal is defined to include both $W^{ \pm} W^{ \pm} \gamma$ and $W^{ \pm} Z \gamma$ production. Events are selected by requiring an isolated lepton $(\mu, e)$ and missing energy $E_{\mathrm{T}}^{\text {miss }}>35 \mathrm{GeV}$ to form a transverse mass above $30 \mathrm{GeV}$, and an isolated photon with $p_{\mathrm{T}}>30 \mathrm{GeV}$, and two jets forming a invariant mass consistent with a hadronically decaying $W^{ \pm}$or $Z$ boson, $70 \mathrm{GeV}<m_{j j}<100 \mathrm{GeV}$. To reduce background from top quarks the jets must not be $b$-tagged. The dominant background is from $W^{ \pm} \gamma+$ jets production and modelled with simulation while the normalisation is taken from data in a control region. After all selection cuts CMS observes 183 (139) candidates in the muon (electron) 
channel, while the expected signal plus background events is $194.2 \pm 11.5$ (147.9 \pm 10.7$)$, leading to an upper limit of the production cross section of $311 \mathrm{fb}$ at $95 \%$ CL. CMS uses the photon transverse energy spectrum to set limits on anomalous QGC shown in Figure 24 to extract limits on dimension 6 EFT couplings $a_{0}^{W} / \Lambda^{2}$ and $a_{C}^{W} / \Lambda^{2}$, describing $W W \gamma \gamma$ interactions, and $\kappa_{0}^{W} / \Lambda^{2}$ and $\kappa_{C}^{W} / \Lambda^{2}$, describing $W W Z \gamma$ interactions. The 95\%CL limits are reported in the range of $12 \mathrm{TeV}^{-2}$ to $34 \mathrm{TeV}^{-2}$. Limits on the dimension 8 EFT couplings $f_{T, 0} / \Lambda^{4}, f_{M, 0} / \Lambda^{4}, f_{M, 1} / \Lambda^{4}, f_{M, 2} / \Lambda^{4}$, and $f_{M, 3} / \Lambda^{4}$ are also reported in the range of $25 \mathrm{TeV}^{-4}$ for $f_{T, 0} / \Lambda^{4}$ and $40-131 \mathrm{TeV}^{-4}$ for $f_{M, i} / \Lambda^{4}$.

ATLAS searched for $W^{ \pm} W^{ \pm} \gamma$ production in the fully leptonic decay channel and for $W^{ \pm} V \gamma$ production in the semi-leptonic decay channel [156]. In the fully leptonic channel the mixed flavour signature $e \nu \mu \nu \gamma$ is selected, as the same flavour channel is dominated by Drell-Yan production. Signal events are required to have two opposite sign mixed flavour leptons $(e, \mu)$ with $p_{\mathrm{T}}>20 \mathrm{GeV}$, one photon with $p_{\mathrm{T}}>15 \mathrm{GeV}$, and $E_{\mathrm{T}}^{\text {miss }}>15 \mathrm{GeV}$. Events with a third lepton or a jet are vetoed to reduce the background from $W^{+} W^{-}, W^{ \pm} Z$, and top quark production. After all selection cuts a total of 26 events are observed, with $12.2 \pm 1.1$ signal events over $15.1 \pm 4.1$ background events expected, corresponding to an observed significance of $1.4 \sigma$ (1.6 $\sigma$ expected). For the semileptonic analysis the event selection requires one lepton, at least two jets, and least one photon, and missing transverse momentum. Events with the invariant mass of electron and photon close to the $Z$ mass or with $b$-tagged jets are vetoed to reduce the background from $Z+$ jets and top quark events. A total of 490 (599) events are found with a background expectation of $552 \pm 38(621 \pm 31)$ and signal expectation of $14 \pm 2(18 \pm 2)$ in the electron (muon) channel. Upper production cross-sections are derived at the 95\% CL for the semi-leptonic channel of 6 $\mathrm{fb}$ with $8.4_{-2.4}^{+3.4} \mathrm{fb}$ expected, and for the fully leptonic channel of $3.7 \mathrm{fb}$ with $2.1_{-0.6}^{+0.9}$ expected. The SM prediction for comparison is $2.0 \pm 0.1 \mathrm{fb}$ for the fully leptonic channel and $2.3 \pm 0.1 \mathrm{fb}$ for the semileptonic channel. To extract anomalous QGC the fiducial phase space is further restricted by raising the photon transverse energy requirement to $120 \mathrm{GeV}(200 \mathrm{GeV})$ in the fully leptonic (semi-leptonic) analysis to optimise the sensitivity, and the channels are combined. Limits are set on $f_{M, 0,1,2,3,4,5,6,7} / \Lambda^{4}$, $f_{T, 0,1,2,5,6,7} / \Lambda^{4}$ in the range of $0.1 \cdot 10^{3} \mathrm{TeV}^{-4}$ to $4.3 \cdot 10^{3} \mathrm{TeV}^{-4}$, roughly in agreement with the expected sensitivity. The sensitivity is not as high as the CMS analysis discussed above, as an optimised fiducial region is used instead of a binned distribution in the transverse energy of the photon.

\subsubsection{The $W^{ \pm} W^{ \pm} W^{\mp}$ process}

The production of three massive vector bosons is the most challenging channel amongst tri-boson final states due to its small production cross section with three on shell massive vector bosons. A measurement of this channel will start to become accessible with analysis of the full $13 \mathrm{TeV}$ dataset. Both experiments have analysis efforts ongoing and first preliminary results have been presented, however at the time of writing no published results are available at $\sqrt{s}=13 \mathrm{TeV}$. Results exist for the $8 \mathrm{TeV}$ data-set. An ATLAS analysis uses the fully leptonic $(\ell \nu \ell \nu \ell \nu)$ and semi-leptonic $(\ell \nu \ell \nu q q)$ decay channel of $W^{ \pm} W^{ \pm} W^{\mp}$ production to search for this process [158]. To select $\ell \nu \ell \nu \ell \nu$ candidates exactly three lepton, electrons or muons, with $p_{\mathrm{T}}>20 \mathrm{GeV}$, at most one jet and no $b$-tagged jet is required, and the three lepton system and $E_{\mathrm{T}}^{\mathrm{miss}}$ direction must be separated in the transverse plane.

The semi-leptonic channel selects $\ell \nu \ell \nu j j$ events by requiring exactly two same sign leptons with $p t>30 \mathrm{GeV}$ and $m_{l l}>40 \mathrm{GeV}, E_{\mathrm{T}}^{\text {miss }}$ above $55 \mathrm{GeV}$ (except for the muonic channel), and at least two jets, and no $b$-tagged jet. In the case of $e^{ \pm} e^{ \pm}$the the di-electron invariant mass $m_{e e}$ must satisfy $m_{e e}<80 \mathrm{GeV}$ and $m_{e e}>100 \mathrm{GeV}$ to reduce the background from events containing $Z \rightarrow e^{+} e^{-}$ where one electron charge is misidentified. Events with additional leptons are removed to reduce the background from di-boson processes. Combining the $\ell \nu \ell \nu \ell \nu$ and $\ell \nu \ell \nu j j$ channel a signal significance of $0.96 \sigma$ is observed with $1.05 \sigma$ expected. Figure 25 shows the invariant tri-lepton mass distribution for the fully leptonic channel and the sum of transverse momenta spectrum for the semi-leptonic channel, together with signal and background predictions. ATLAS uses these distributions to extract limits 
on two dimension 8 EFT parameters, and constrains $f_{S, 0} / \Lambda^{4}$ and $f_{S, 1} / \Lambda^{4}$ at the $95 \%$ CL to -0.13 . $10^{4} \mathrm{TeV}^{-4}<f_{S, 0} / \Lambda^{4}<0.18 \cdot 10^{4} \mathrm{TeV}^{-4},-0.21 \cdot 10^{4} \mathrm{TeV}^{-4}<f_{S, 1} / \Lambda^{4}<0.27 \cdot 10^{4} \mathrm{TeV}^{-4}$.

\section{Summary}

The successful operation of the LHC has provided the ATLAS and CMS collaborations with a rich data-set of $p p$ collisions at $7 \mathrm{TeV}$ and $8 \mathrm{TeV}$ in run-1, and $13 \mathrm{TeV}$ in run-2. Already with run-1 data the ATLAS and CMS collaborations have explored all inclusive di-boson final-states $\left(W^{+} W^{-}, W^{ \pm} Z\right.$, $\left.Z Z, W^{ \pm} \gamma, Z \gamma\right)$. The set of observables is increasing as the analyses mature, total and fiducial cross section, unfolded differential distributions of kinematic variables and lately also polarisation fractions provide a rich set of legacy measurement that can be compared to phenomenology. An exciting part of the LHC physics programme is the study of electroweak production of vector bosons via exclusive processes. With run-1 data evidence was obtained for $W^{ \pm} W^{ \pm}$and $Z \gamma$ vector boson scattering processes for the first time. Also, tri-boson processes have been observed in the $V \gamma \gamma$ channel, but other states with two $(V V \gamma)$ or three $(V V V)$ heavy vector bosons in the final were statistically limited with the run-1 data-set.

Also the theoretical developments have kept pace with the experimental advancements in precision and targeted final states. The advent of automated calculation tools allowed a rapid expansion of available prediction with higher order correction in $\alpha$ and $\alpha_{s}$.

With the successful completion of run-2, the analysis effort is in full swing and updated results can be expected to emerge before the start of run-3. First results at $\sqrt{s}=13 \mathrm{TeV}$ gather evidence for hitherto unobserved VBS processes $\left(W^{ \pm} Z\right)$ and tri-boson final states. With the increased integrated luminosity and collision energy of the run-2 data-set evidence for $V V \gamma$ and $V V V$ production should be obtainable.

The non-abelian structure of the electroweak gauge sector of the standard model has been tested with di-boson, tri-boson and VBS processes by EFT and anomalous gauge couplings. The limits obtained the most precise available to date, surpassing previous constraints from the Tevatron and LEP experiments. These generic tests of the validity of the standard model yield so far no indication of necessity for new physics beyond the standard model.

With the completion of run-2 analysis, a combination of different channels and results between experiments should further enhance the reach of EFT constraints. The common EFT framework encompassing vector bosons and the scalar Higgs allows to powerfully combine the Higgs and the electroweak sector to look for effects of physics beyond the standard model. The enhanced statistics may allow to study angular distributions and polarisation fractions not only in inclusive but also in exclusive diboson states. The accessibility of kinematic variables to distinguish $\mathrm{CP}$ violating anomalous coupling parameters will be a major step forward. 

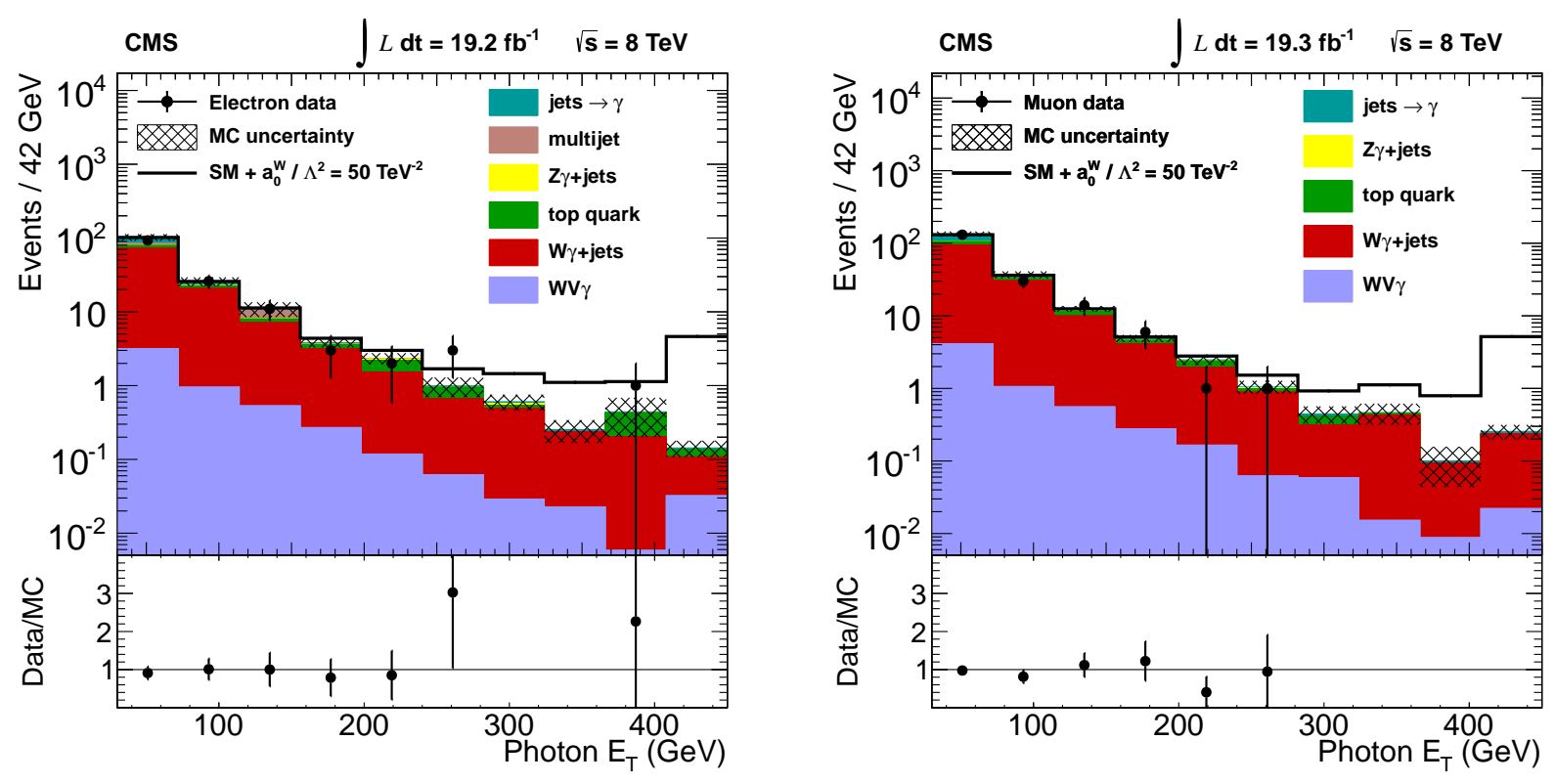

Figure 24: Photon transverse energy distribution of $W V \gamma$ candidates in the $\sqrt{s}=8 \mathrm{TeV}$ data-set reported by CMS [157].
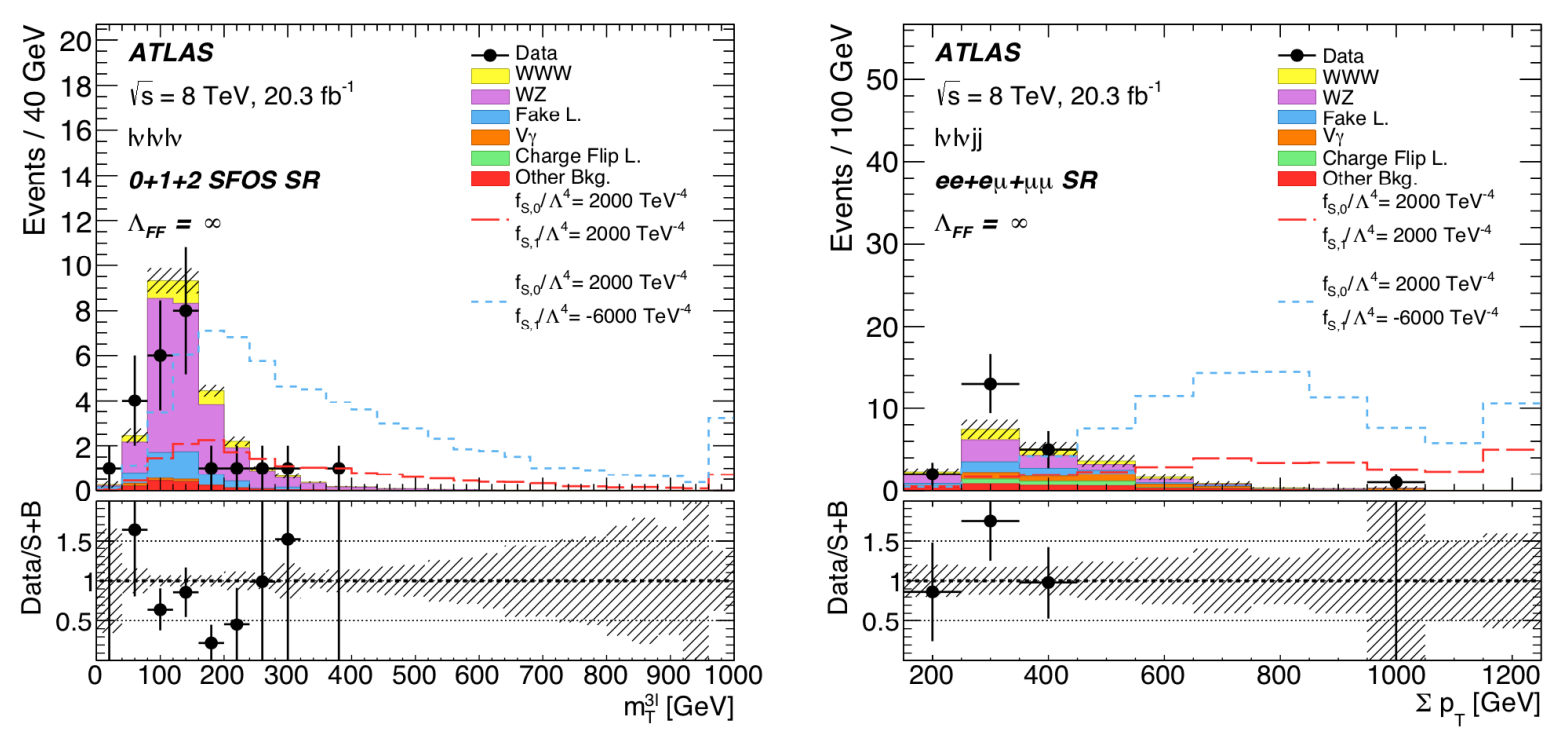

Figure 25: Distribution of the tri-lepton invariant mass $m_{l l l}$ (left) in the $\ell \nu \ell \nu \ell \nu$ channel and the distribution of the sum of transverse momenta $\Sigma p_{\mathrm{T}}$ in the $\ell \nu \ell \nu j j$ channel from the ATLAS $8 \mathrm{TeV} W^{ \pm} W^{ \pm} W^{\mp}$ search [158]. Data with simulated background and signal contributions are shown. The lower plot shows the ratio of data over prediction, with the hashed band indicating the systematic uncertainty. Also shown are signal predictions with assumed anomalous QGC couplings. 


\section{References}

[1] Schael S et al. (DELPHI, OPAL, LEP Electroweak, ALEPH, L3) 2013 Phys. Rept. 532 119-244 (Preprint 1302.3415)

[2] Abazov V et al. (The D0 Collaboration) 2012 Phys. Rev. D 85(11) 112005 URL https://link.aps.org/doi/10.1103/PhysRevD.85.112005

[3] Aaltonen T et al. (CDF) 2012 Phys. Rev. D86 031104 (Preprint 1202.6629)

[4] Evans L and Bryant P 2008 JINST 3 S08001

[5] ALEPH Collaboration, CDF Collaboration, D0 Collaboration, DELPHI Collaboration, L3 Collaboration, OPAL Collaboration, SLD Collaboration, LEP Electroweak Working Group, Tevatron Electroweak Working Group and SLD electroweak heavy flavour group 2010 ArXiv e-prints (Preprint 1012.2367)

[6] Aad G et al. 2012 Physics Letters $B \begin{array}{rlllllll}\mathbf{7 1 6} & 1 & - & 29 & \text { ISSN } & \text { 0370-2693 } & \text { URL }\end{array}$ http://www.sciencedirect.com/science/article/pii/S037026931200857X

[7] Chatrchyan $\mathrm{S}$ et al. 2012 Physics Letters $B \begin{array}{rllllll}\mathbf{7 1 6} & 30 & - & 61 & \text { ISSN } & 0370-2693 & \text { URL }\end{array}$ http://www.sciencedirect.com/science/article/pii/S0370269312008581

[8] Aad G et al. (ATLAS) 2016 Phys. Rev. D93 112002 (Preprint 1604.05232)

[9] Sirunyan A M et al. (CMS) 2017 JHEP 10072 (Preprint 1704.00366)

[10] Green D R, Meade P and Pleier M A 2017 Rev. Mod. Phys. 89035008 (Preprint 1610.07572)

[11] Bruce R et al. 2016 MOAM5P50. 7 p URL https://cds.cern.ch/record/2201447

[12] Steerenberg R 2018 LHC Report: Another run is over and LS2 has just begun? URL https://home.cern/news/news/accelerators/lhc-report-another-run-over-and-1s2-has-just-be

[13] Hagiwara K, Peccei R, Zeppenfeld D and Hikasa K 1987 Nuclear Physics B 282253 - 307 ISSN 0550-3213 URL http://www.sciencedirect.com/science/article/pii/0550321387906857

[14] Agashe K, Davoudiasl H, Perez G and Soni A 2007 Phys. Rev. D76 036006 (Preprint hep-ph/0701186)

[15] Altarelli G, Mele B and Ruiz-Altaba M 1989 Z. Phys. C45 109 [Erratum: Z. Phys.C47,676(1990)]

[16] Dorigo T 2018 Prog. Part. Nucl. Phys. 100 211-261 (Preprint 1802.00354)

[17] Weinberg S 1979 Physica A96 327-340

[18] Weinberg S 1980 Phys. Lett. B91 51-55

[19] Georgi H 1993 Ann. Rev. Nucl. Part. Sci. 43 209-252

[20] Weinberg S 1979 Phys. Rev. Lett. 43(21) 1566-1570 URL https://link.aps.org/doi/10.1103/PhysRevLett. 43.1566

[21] Gaemers K J F and Gounaris G J 1979 Zeitschrift für Physik C Particles and Fields 1 259-268 ISSN 1431-5858 URL https://doi.org/10.1007/BF01440226 
[22] Gounaris G et al. 1996 Triple gauge boson couplings AGS / RHIC Users Annual Meeting Upton, New York, June 15-16, 1995 (Preprint hep-ph/9601233) URL http: //alice.cern. ch/format/showfull?sysnb=0215385

[23] Hagiwara K, Ishihara S, Szalapski R and Zeppenfeld D 1993 Phys. Rev. D 48(5) 2182-2203 URL http://link.aps.org/doi/10.1103/PhysRevD.48.2182

[24] Hagiwara K, Ishihara S, Szalapski R and Zeppenfeld D 1992 Physics Letters B 283353 - 359 ISSN 0370-2693 URL http://www.sciencedirect.com/science/article/pii/037026939290031X

[25] Gounaris G J, Layssac J and Renard F M 2000 Phys. Rev. D 62(7) 073013 URL https://link.aps.org/doi/10.1103/PhysRevD.62.073013

[26] Baur U and Rainwater D 2000 Phys. Rev. D $\mathbf{6 2 ( 1 1 )} 113011$ URL https://link.aps.org/doi/10.1103/PhysRevD.62.113011

[27] Baur U and Berger E L 1993 Phys. Rev. D 47(11) 4889-4904 URL http://link.aps.org/doi/10.1103/PhysRevD.47.4889

[28] Baur U and Zeppenfeld D 1988 Physics Letters B 201383 - 389 ISSN 0370-2693 URL http://www.sciencedirect.com/science/article/pii/0370269388911604

[29] Aihara H et al. 1995 488-546 (Preprint hep-ph/9503425)

[30] Aad G et al. (ATLAS Collaboration) 2013 Phys.Rev. D87 112001 (Preprint 1210.2979)

[31] Chatrchyan S et al. (CMS Collaboration) 2013 Eur.Phys.J. C73 2610 (Preprint 1306.1126)

[32] Degrande C, Greiner N, Kilian W, Mattelaer O, Mebane H, Stelzer T, Willenbrock S and Zhang C 2013 Annals Phys. 335 21-32 (Preprint 1205.4231)

[33] De Rjula A, Gavela M, Hernandez P and Mass E 1992 Nuclear Physics B 3843 - 58 ISSN 0550-3213 URL http://www.sciencedirect.com/science/article/pii/055032139290460S

[34] Hagiwara K, Ishihara S, Szalapski R and Zeppenfeld D 1993 Phys. Rev. D48 2182-2203

[35] Wudka J 1994 Int. J. Mod. Phys. A9 2301-2362 (Preprint hep-ph/9406205)

[36] Ellison J and Wudka J 1998 Ann. Rev. Nucl. Part. Sci. 48 33-80 (Preprint hep-ph/9804322)

[37] Buchmüller W and Wyler D 1986 Nuclear Physics B 268621 - 653 ISSN 0550-3213 URL http://www.sciencedirect.com/science/article/pii/0550321386902622

[38] Ellis J, Murphy C W, Sanz V and You T 2018 JHEP 06146 (Preprint 1803.03252)

[39] Grzadkowski B, Iskrzynski M, Misiak M and Rosiek J 2010 JHEP 10085 (Preprint 1008.4884)

[40] Giudice G, Grojean C, Pomarol A and Rattazzi R 2007 JHEP 06045 (Preprint hep-ph/0703164)

[41] Contino R, Ghezzi M, Grojean C, Muhlleitner M and Spira M 2013 JHEP 07035 (Preprint 1303.3876)

[42] Falkowski A, Fuks B, Mawatari K, Mimasu K, Riva F and Sanz V 2015 Eur. Phys. J. C75 583 (Preprint 1508.05895)

[43] Brivio I and Trott M 2017 (Preprint 1706.08945) 
[44] Denner A, Dittmaier S, Roth M and Wackeroth D 2001 Off-shell W pair production: Universal versus nonuniversal corrections Proceedings, 5th International Symposium on Radiative Corrections - RADCOR 2000 (Preprint hep-ph/0101257) URL http://www.slac.stanford.edu/econf/C000911/

[45] Gehrmann T et al. 2014 Phys. Rev. Lett. 113212001 (Preprint 1408.5243)

[46] Grazzini M, Kallweit S, Pozzorini S, Rathlev D and Wiesemann M 2016 JHEP 08140 (Preprint 1605.02716)

[47] Biedermann B et al. 2016 JHEP 06065 (Preprint 1605.03419)

[48] Kallweit S, Lindert J M, Pozzorini S and Schönherr M 2017 JHEP 11120 (Preprint 1705.00598)

[49] Cascioli F et al. 2014 Phys. Lett. B735 311-313 (Preprint 1405.2219)

[50] Kallweit S and Wiesemann M 2018 (Preprint 1806.05941)

[51] Biedermann B, Denner A, Dittmaier S, Hofer L and Jäger B 2016 Phys. Rev. Lett. 116161803 (Preprint 1601.07787)

[52] Grazzini M, Kallweit S, Rathlev D and Wiesemann M 2016 Phys. Lett. B761 179-183 (Preprint 1604.08576)

[53] Biedermann B, Denner A and Hofer L 2017 JHEP 10043 (Preprint 1708.06938)

[54] Caola F, Melnikov K, Röntsch R and Tancredi L 2016 Phys. Lett. B754 275-280 (Preprint 1511.08617)

[55] Caola F, Melnikov K, Röntsch R and Tancredi L 2015 Phys. Rev. D92 094028 (Preprint 1509.06734)

[56] Biedermann B, Denner A and Pellen M 2017 JHEP 10124 (Preprint 1708.00268)

[57] Schönherr M 2018 JHEP 07076 (Preprint 1806.00307)

[58] Campanario F, Hankele V, Oleari C, Prestel S and Zeppenfeld D 2008 Phys. Rev. D78 094012 (Preprint 0809.0790)

[59] Greiner N and Schönherr M 2018 JHEP 01079 (Preprint 1710.11514)

[60] Shen Y B, Zhang R Y, Ma W G, Li X Z, Zhang Y and Guo L 2015 JHEP 10186 [Erratum: JHEP10,156(2016)] (Preprint 1507.03693)

[61] Wang H et al. 2016 J. Phys. G43 115001 (Preprint 1610.05876)

[62] Aaboud M et al. (ATLAS) 2016 Phys. Lett. B762 1-22 (Preprint 1606.04017)

[63] Nason P 2004 JHEP 11040 (Preprint hep-ph/0409146)

[64] Frixione S, Nason P and Oleari C 2007 JHEP 11070 (Preprint 0709.2092)

[65] Frixione S and Webber B R 2002 JHEP 06029 (Preprint hep-ph/0204244)

[66] Frixione S, Nason P and Webber B R 2003 JHEP 08007 (Preprint hep-ph/0305252) 
[67] Hamilton K, Nason P, Re E and Zanderighi G 2013 JHEP 10222 (Preprint 1309.0017)

[68] Alioli S, Bauer C W, Berggren C, Tackmann F J and Walsh J R 2015 Phys. Rev. D92 094020 (Preprint 1508.01475)

[69] Re E, Wiesemann M and Zanderighi G 2018 (Preprint 1805.09857)

[70] Alwall J, Frederix R, Frixione S, Hirschi V, Maltoni F, Mattelaer O, Shao H S, Stelzer T, Torrielli $\mathrm{P}$ and Zaro M 2014 JHEP 07079 (Preprint 1405.0301)

[71] Frederix R, Frixione S, Hirschi V, Pagani D, Shao H S and Zaro M 2018 JHEP 07185 (Preprint 1804.10017)

[72] Melia T, Nason P, Rontsch R and Zanderighi G 2011 Eur. Phys. J. C71 1670 (Preprint $1102.4846)$

[73] Jager B and Zanderighi G 2011 JHEP 11055 (Preprint 1108.0864)

[74] Jager B and Zanderighi G 2013 JHEP 04024 (Preprint 1301.1695)

[75] Nason P and Zanderighi G 2014 Eur. Phys. J. C74 2702 (Preprint 1311.1365)

[76] Jäger B, Karlberg A and Zanderighi G 2014 JHEP 03141 (Preprint 1312.3252)

[77] Schönherr M 2018 The European Physical Journal C 78 119 ISSN 1434-6052 URL https://doi.org/10.1140/epjc/s10052-018-5600-z

[78] Baglio J et al. 2011 (Preprint 1107.4038)

[79] Aad G et al. (ATLAS Collaboration) 2008 JINST 3 S08003

[80] Chatrchyan S et al. (CMS Collaboration) 2008 JINST 3 S08004

[81] Froidevaux D and Sphicas P 2006 Annual Review of Nuclear and Particle Science 56 375-440 (Preprint https://doi.org/10.1146/annurev.nucl.54.070103.181209) URL https://doi.org/10.1146/annurev.nucl.54.070103.181209

[82] Aad G et al. (ATLAS) 2014 Eur. Phys. J. C74 2941 (Preprint 1404.2240)

[83] Khachatryan V et al. (CMS) 2015 JINST 10 P06005 (Preprint 1502.02701)

[84] Aad G et al. (ATLAS) 2016 Eur. Phys. J. C76 292 (Preprint 1603.05598)

[85] Chatrchyan S et al. (CMS) 2012 JINST 7 P10002 (Preprint 1206.4071)

[86] Aad G et al. (ATLAS) 2017 Eur. Phys. J. C77 490 (Preprint 1603.02934)

[87] Ellis S D and Soper D E 1993 Phys. Rev. D48 3160-3166 (Preprint hep-ph/9305266)

[88] Dokshitzer Y L, Leder G D, Moretti S and Webber B R 1997 JHEP 08001 (Preprint hep-ph/9707323)

[89] Sirunyan A M et al. (CMS) 2017 JINST 12 P10003 (Preprint 1706.04965)

[90] Aaboud M et al. 2017 The European Physical Journal C 77466 ISSN 1434-6052 URL https://doi.org/10.1140/epjc/s10052-017-5031-2 
[91] Aaboud M et al. 2018 The European Physical Journal C 78903 ISSN 1434-6052 URL https ://doi.org/10.1140/epjc/s10052-018-6288-9

[92] Khachatryan V et al. (CMS) 2015 JINST 10 P02006 (Preprint 1411.0511)

[93] LEP Electroweak Gauge-Couplings Group 2002 A combination of preliminary results on gauge boson couplings measured by the lep experiments URL http://lepewwg . web. cern. ch/lepww/tgc/summer02

[94] Kotwal A V, Schellman H and Sekaric J 2015 Int. J. Mod. Phys. A30 1541004 (Preprint 1409.5163)

[95] Chatrchyan S et al. (CMS Collaboration) 2014 Phys.Rev. D89 092005 (Preprint 1308.6832)

[96] Alcaraz J et al. (ALEPH, DELPHI, L3, OPAL, LEP Electroweak Working Group) 2006 (Preprint hep-ex/0612034)

[97] Robson A (CDF, D0) 2012 EPJ Web Conf. 2806001 (Preprint 1201.4771)

[98] Chatrchyan S et al. (CMS Collaboration) 2013 JHEP 1310164 (Preprint 1309.1117)

[99] Aaboud M et al. (ATLAS) 2018 JHEP 12010 (Preprint 1810.04995)

[100] Aad G et al. (ATLAS Collaboration) 2013 Phys.Rev. D87 112003 (Preprint 1302.1283)

[101] Khachatryan V et al. (CMS Collaboration) 2015 JHEP 1504164 (Preprint 1502.05664)

[102] Khachatryan V et al. (CMS) 2016 Phys. Lett. B760 448-468 (Preprint 1602.07152)

[103] Aad G et al. (ATLAS) 2016 JHEP 09029 (Preprint 1603.01702)

[104] Khachatryan V et al. (CMS) 2016 Eur. Phys. J. C76 401 (Preprint 1507.03268)

[105] Aad G et al. (ATLAS Collaboration) 2012 Eur.Phys.J. C72 2173 (Preprint 1208.1390)

[106] Khachatryan V et al. (CMS) 2017 Eur. Phys. J. C77 236 (Preprint 1609.05721)

[107] Aad G et al. (ATLAS) 2016 Phys. Rev. D93 092004 (Preprint 1603.02151)

[108] Aaboud M et al. (ATLAS) 2019 (Preprint 1902.05759)

[109] Sirunyan A M et al. (CMS) 2019 JHEP 04122 (Preprint 1901.03428)

[110] Groote S, Korner J G and Tuvike P 2012 Eur. Phys. J. C72 2177 (Preprint 1204.5295)

[111] Gounaris G J, Layssac J and Renard F M 2000 Phys. Rev. D62 073013 (Preprint hep-ph/0003143)

[112] Grazzini M, Kallweit S and Rathlev D 2015 Phys. Lett. B750 407-410 (Preprint 1507.06257)

[113] Olive K A et al. (Particle Data Group) 2014 Chin. Phys. C38 090001

[114] Aad G et al. (ATLAS Collaboration) 2013 JHEP 1303128 (Preprint 1211.6096)

[115] Aaboud M et al. (ATLAS) 2017 JHEP 01099 (Preprint 1610.07585)

[116] Aaboud M et al. (ATLAS) 2018 Phys. Rev. D97 032005 (Preprint 1709.07703) 
[117] Chatrchyan S et al. (CMS Collaboration) 2013 JHEP 1301063 (Preprint 1211.4890)

[118] Khachatryan V et al. (CMS) 2015 Phys. Lett. B740 250-272 (Preprint 1406.0113)

[119] Khachatryan V et al. (CMS) 2015 Eur. Phys. J. C75 511 (Preprint 1503.05467)

[120] Sirunyan A M et al. (CMS) 2018 Eur. Phys. J. C78 165 [Erratum: Eur. Phys. J.C78,no.6,515(2018)] (Preprint 1709.08601)

[121] Campbell J M, Ellis R K and Williams C 2011 JHEP 07018 (Preprint 1105.0020)

[122] Bierweiler A, Kasprzik T and Kühn J H 2013 JHEP 12071 (Preprint 1305.5402)

[123] Baglio J, Ninh L D and Weber M M 2013 Phys. Rev. D88 113005 (Preprint 1307.4331)

[124] Baur U and Rainwater D L 2001 Int. J. Mod. Phys. A16S1A 315-317 (Preprint hep-ph/0011016)

[125] Aad G et al. (ATLAS Collaboration) 2015 JHEP 1501049 (Preprint 1410.7238)

[126] Chatrchyan S et al. (CMS Collaboration) 2013 Eur.Phys.J. C73 2283 (Preprint 1210.7544)

[127] Aaboud M et al. (ATLAS) 2017 Eur. Phys. J. C77 563 (Preprint 1706.01702)

[128] Sirunyan A M et al. (CMS) 2017 Phys. Lett. B772 21-42 (Preprint 1703.06095)

[129] Chatrchyan S et al. (CMS Collaboration) 2014 Eur.Phys.J. C74 2973 (Preprint 1403.3047)

[130] Aad G et al. (ATLAS Collaboration) 2014 JHEP 1404031 (Preprint 1401.7610)

[131] Khachatryan V et al. (CMS Collaboration) 2015 Eur.Phys.J. C75 66 (Preprint 1410.3153)

[132] Khachatryan V et al. (CMS Collaboration) 2015 Phys.Rev.Lett. 114051801 (Preprint 1410.6315)

[133] Aaboud M et al. (ATLAS) 2017 Phys. Rev. D96 012007 (Preprint 1611.02428)

[134] Sirunyan A M et al. (CMS) 2018 Phys. Rev. Lett. 120081801 (Preprint 1709.05822)

[135] Khachatryan V et al. (CMS) 2017 JHEP 06106 (Preprint 1612.09256)

[136] Aaboud M et al. (ATLAS) 2017 JHEP 07107 (Preprint 1705.01966)

[137] Khachatryan V et al. (CMS) 2017 Phys. Lett. B770 380-402 (Preprint 1702.03025)

[138] Sirunyan A M et al. (CMS) 2019 (Preprint 1901.04060)

[139] Aaboud M et al. (ATLAS) 2018 Submitted to: Phys. Lett. (Preprint 1812.09740)

[140] Sirunyan A M et al. (CMS) 2017 Phys. Lett. B774 682-705 (Preprint 1708.02812)

[141] Aaboud M et al. (ATLAS) 2016 Phys. Rev. D94 032011 (Preprint 1607.03745)

[142] Khachatryan V et al. (CMS) 2016 JHEP 08119 (Preprint 1604.04464)

[143] Vermaseren J A M 1983 Nucl. Phys. B229 347-371

[144] Baranov S P, Duenger O, Shooshtari H and Vermaseren J A M 1991 LPAIR: A generator for lepton pair production In *Hamburg 1991, Proceedings, Physics at HERA, vol. 3* 1478-1482. (see HIGH ENERGY PHYSICS INDEX 30 (1992) No. 12988) 
[145] Bahr M et al. 2008 Eur. Phys. J. C58 639-707 (Preprint 0803.0883)

[146] Zhu B, Govoni P, Mao Y, Mariotti C and Wu W 2011 Eur. Phys. J. C71 1514 (Preprint 1010.5848)

[147] Aad G et al. (ATLAS Collaboration) 2014 Phys.Rev.Lett. 113141803 (Preprint 1405.6241)

[148] Alioli S, Nason P, Oleari C and Re E 2010 JHEP 06043 (Preprint 1002.2581)

[149] Jager B, Oleari C and Zeppenfeld D 2009 Phys. Rev. D80 034022 (Preprint 0907.0580 )

[150] Melia T, Melnikov K, Rontsch R and Zanderighi G 2010 JHEP 12053 (Preprint 1007.5313)

[151] Alboteanu A, Kilian W and Reuter J 2008 JHEP 11010 (Preprint 0806.4145 )

[152] Kilian W, Ohl T and Reuter J 2011 Eur. Phys. J. C71 1742 (Preprint 0708.4233)

[153] Moretti M, Ohl T and Reuter J 2001 1981-2009 (Preprint hep-ph/0102195)

[154] Eboli O J P, Gonzalez-Garcia M C and Mizukoshi J K 2006 Phys. Rev. D74 073005 (Preprint hep-ph/0606118)

[155] Aad G et al. (ATLAS) 2015 Phys. Rev. Lett. 115031802 (Preprint 1503.03243)

[156] Aaboud M et al. (ATLAS) 2017 Eur. Phys. J. C77 646 (Preprint 1707.05597)

[157] Chatrchyan S et al. (CMS) 2014 Phys. Rev. D90 032008 (Preprint 1404.4619)

[158] Aaboud M et al. (ATLAS) 2017 Eur. Phys. J. C77 141 (Preprint 1610.05088) 